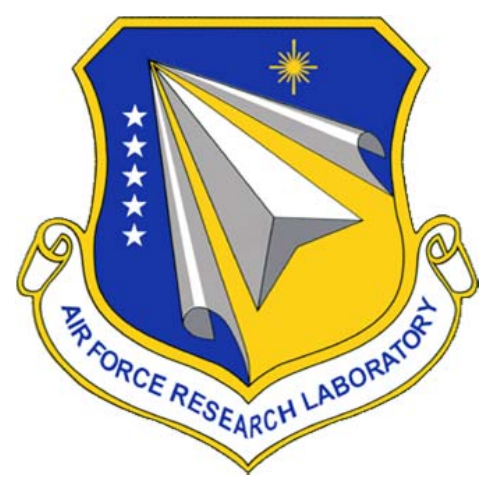

AFRL-AFOSR-UK-TR-2014-0024

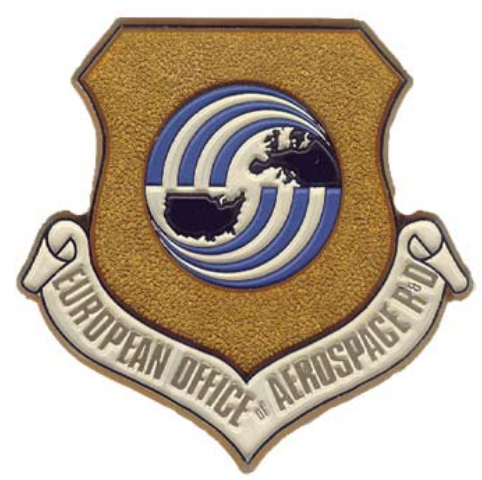

Additive Layer Manufacturing of Biologically Inspired Short Fibre Reinforced Composites

\author{
Dr. Richard S. Trask \\ Mr. Mark Hazzard \\ Mr. Tom Llewellyn-Jones \\ The University of Bristol \\ Advanced Composites Centre for Innovation and Science (ACCIS) \\ Senate House \\ Bristol BS8 1TH United Kingdom
}

EOARD Grant 13-3003

Report Date: March 2014

Final Report from 1 January 2013 to 31 March 2014

Distribution Statement A: Approved for public release distribution is unlimited.
Air Force Research Laboratory
Air Force Office of Scientific Research
European Office of Aerospace Research and Development
Unit 4515, APO AE 09421-4515 


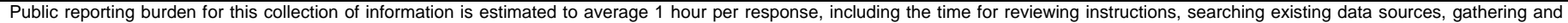

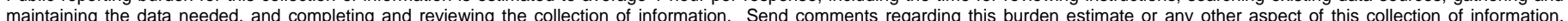

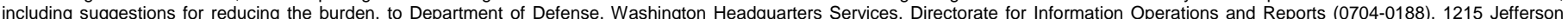

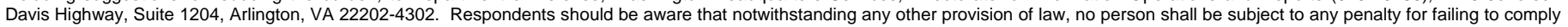
with a collection of information if it does not display a currently valid OMB control number.

PLEASE DO NOT RETURN YOUR FORM TO THE ABOVE ADDRESS.
1. REPORT DATE (DD-MM-YYYY)
30 March 2014
Final Report
3. DATES COVERED (From - To)
2. REPORT TYPE
1 January $2013-31$ March 2014

4. TITLE AND SUBTITLE

Additive Layer Manufacturing of Biologically Inspired Short Fibre Reinforced Composites 5a. CONTRACT NUMBER

FA8655-13-1-3003

5b. GRANT NUMBER

Grant 13-3003

5c. PROGRAM ELEMENT NUMBER

$61102 \mathrm{~F}$

6. AUTHOR(S)

5d. PROJECT NUMBER

Dr. Richard S. Trask

Mr. Mark Hazzard

5d. TASK NUMBER

Mr. Tom Llewellyn-Jones

5e. WORK UNIT NUMBER

7. PERFORMING ORGANIZATION NAME(S) AND ADDRESS(ES)

The University of Bristol

Advanced Composites Centre for Innovation and Science (ACCIS)

Senate House

Bristol BS8 1TH United Kingdom

9. SPONSORING/MONITORING AGENCY NAME(S) AND ADDRESS(ES)

EOARD

Unit 4515

APO AE 09421-4515
8. PERFORMING ORGANIZATION REPORT NUMBER

N/A

10. SPONSOR/MONITOR'S ACRONYM(S)

AFRL/AFOSR/IOE (EOARD)

11. SPONSOR/MONITOR'S REPORT NUMBER(S)

AFRL-AFOSR-UK-TR-2014-0024

\section{DISTRIBUTION/AVAILABILITY STATEMENT}

Distribution A: Approved for public release; distribution is unlimited.

13. SUPPLEMENTARY NOTES

14. ABSTRACT

This project provided fundamental understanding towards novel additive layer manufacturing approaches for highly-ordered, short-fiber architectures. The fabrication challenge is a critical hurdle to the realization of complex, multi-scale architectures inspired by nature for improving the mechanical and functional properties of engineered materials. The study was advanced on two fronts: (1) development of new hierarchical architecture based on $\mathrm{ZnO}$ nanorods grown on glass fibers and coated with tetraethyl orthosilicate (TEOS) to promote self-assembly; and (2) exploitation of ultrasonic manipulation for controlling orientation and distribution of reinforcing short fibers. The ZnO/TEOS nanorods were shown to preserve mechanical properties of the base fibers while also displaying some healing capability for defects. For the ultrasonic manipulation, both micro-scale and nano-scale reinforcements were studied using a new prototype assembly system developed for the project. Enhancement of stiffness and strength properties in aligned directions was observed despite low volume fraction of reinforcement.

\section{SUBJECT TERMS}

EOARD, camouflage, biomimetics, active materials, adaptive, multi-scale structural mechanics, metamaterials, finite elements 16. SECURITY CLASSIFICATION OF:
a. REPORT UNCLAS b. ABSTRACT UNCLAS c. THIS PAGE

UNCLAS
17. LIMITATION OF ABSTRACT

SAR
18, NUMBER OF PAGES

89 19a. NAME OF RESPONSIBLE PERSON

Lt Col Randall Pollak

19b. TELEPHONE NUMBER (Include area code) +44 1895 616115, DSN 314-235-6115 


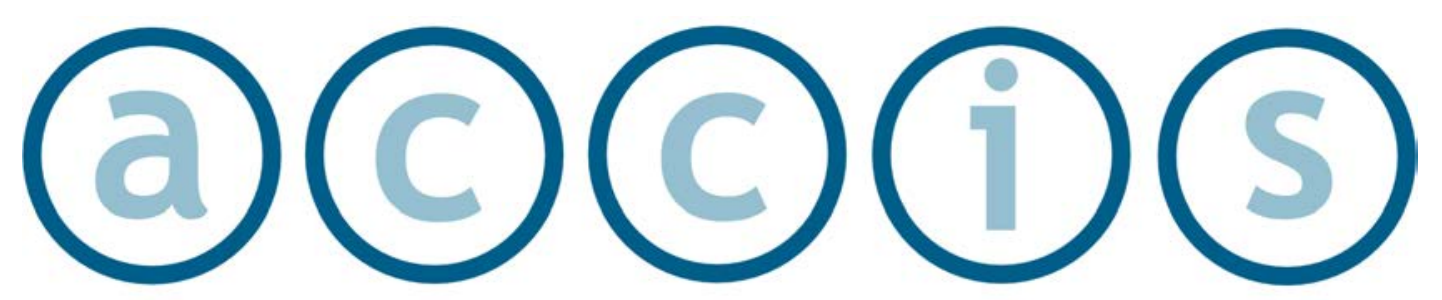

ADVANCED COMPOSITES CENTRE FOR INNOVATION \& SCIENCE

\title{
Additive Layer Manufacturing Of Biologically Inspired Short Fibre Reinforced Composites
}

\author{
Dr. R.S. Trask, Mr Mark Hazzard and \\ Mr Tom Llewellyn-Jones
}

Advanced Composites Centre for Innovation and Science (ACCIS)

University of Bristol

University Walk

Bristol BS8 1TR, UK

March 2014

R.S.Trask@Bristol.ac.uk

Distribution A: Approved for public release; distribution is unlimited. 


\section{Executive Summary}

The scientific vision for this EOARD/AFRL funded project is the development of a novel and highly ordered complex architecture fibrous material for additive layer manufacturing. Using novel chemistry for the generation of new hierarchical fibre architectures and controlled field-effect assembly, functionally graded, stiffness modulated architectures, analogous to those found in nature, are synthesised to realise enhanced mechanical performance, multi-dimensional composite structures.

This report provides the detailed experimental investigations into the generation of Zinc oxide $(\mathrm{ZnO})$ nanorods grown in aqueous solutions onto the surface of E-glass short fibres (approximately $3 \mathrm{~mm}$ in length), and a continuous borosilicate glass fibre $60 \mathrm{~mm}$ in length. This shows that the nanorods do not compromise the mechanical properties of the fibres and furthermore the TEOS coating could 'heal' defects and cause a slight increase in fibre cross sectional area. In the second half of the study, both theoretical analysis and experimental development were used to evaluate the potential of field gradient technologies at generating (1) micron length scale aligned reinforcement and (2) nano length scale aligned composite materials, with regard to rapid prototyping manufacture of complex fibre constructs. To achieve this, a new type of ultrasonic device was developed, separating the acoustic system from the resin cavity to allow for the easy manufacture of multiple samples. Furthermore, an effective method has been outlined to manufacture and mechanically characterise the micron length scale reinforced thin laminar composites. With an $8 \%$ difference in stiffness between the two principal directions, anisotropy was demonstrated for unidirectionally reinforced discontinuous fibre composites under uniaxial tensile loading. A $43 \%$ improvement in strength was observed for samples tested parallel to the direction of

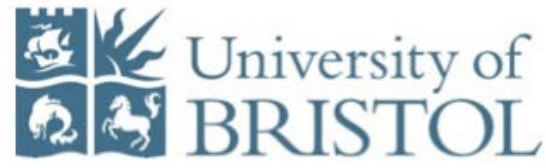


the fibre reinforcement over those strained normal to the fibre direction, despite the relatively low volume percentage of the reinforcement phase. This technique shows great potential for the low cost instantaneous alignment of structural reinforcement to generate the light-weight high performance structures required for future military requirements.

Distribution A: Approved for public release; distribution is unlimited. 


\section{Introduction}

In nature, both material and structure are formed according to the principles of biologically controlled self-assembly, a process defined as the spontaneous and reversible ordering of small molecular building blocks under the influence of noncovalent, static interactions. Further, natural materials have been shown to possess outstanding mechanical properties that are generally accredited to the functional arrangement of short fibrous reinforcement entities over many levels of hierarchy.

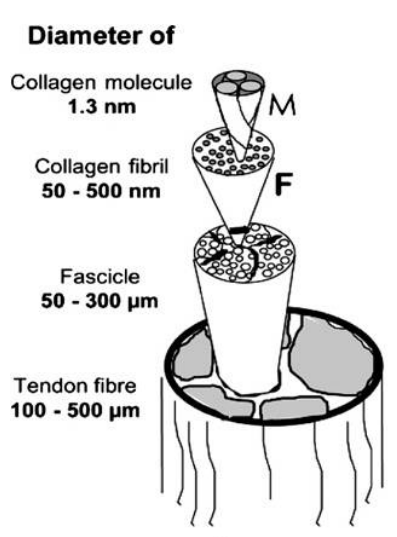

a

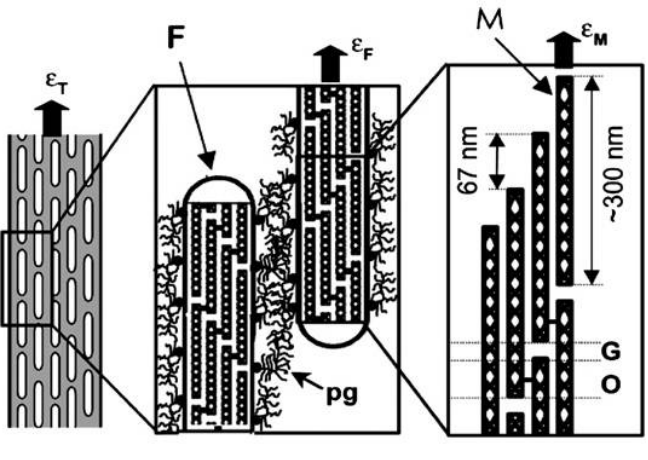

b
C d

Figure 1.1: Simplified representation of the tendon structure, decomposed into four levels of hierarchical organisation. Tendon (a) is made up of several parallel fasciles (b), each containing a large number of collagen fibrils (c), which in turn represent assemblies of highly aligned staggered collagen molecules (d). [1]

There are numerous examples of biological systems with varying degrees of fibrillar organisation that can be found in nature. For the purposes of this study, the specific characteristics of micron-size collagen fibrils have been selected. Collagen is a major constituent of tendon (as well as many other biological tissues) for which, importantly, the following relation has been proven to hold true.

$$
\varepsilon_{T}>\varepsilon_{F}>\varepsilon_{M}
$$

In this equation, $\varepsilon_{\mathrm{T}}, \varepsilon_{\mathrm{F}}$, and $\varepsilon_{\mathrm{M}}$ signify the tensile strains of the whole tendon, a single collagen fibril, and an individual collagen molecule, respectively. While the magnitude of strain is not independent of strain rate, it is still clear that only 
hierarchical effects can cause the above relationship to hold. Specifically, it has been suggested that the overall extension of the tendon may be obtained by summing over fibril elongation and deformation in the matrix due to shear [1]. Similarly, molecular strain and intermolecular shear are thought to constitute total fibril strain [1]. Collagen fibrils are assembled by an axial staggering of individual collagen molecules (Figure 1.1, d) that, when suspended in water, tend to adapt a triple helix conformation. With each helix measuring approximately $300 \mathrm{~nm}$ in length and 1.5 $\mathrm{nm}$ in diameter, molecules are arranged at a periodicity of $67 \mathrm{~nm}$ in the longitudinal direction, a spacing of $35 \mathrm{~nm}$ between ends, and a varying separation of 1.1 to 1.6 $\mathrm{nm}$ between neigbouring strands [1, 2]. A small number of covalent cross-links can be detected between helices. Whole fibrils are typically found to have diameters of the order of several hundred nanometres. Given the above equation, it is postulated that the architectural design features analogous to those of collagen could offer significant improvements in the overall mechanical performance of currently available short fibre composite components. Although relatively good alignment has already been achieved for randomly dispersed particles in solution [3-5], no means are presently accessible allowing reinforcement entities to be ordered in accordance with specific patterns.

The goal of this research is the development of a novel architecture, high performance fibre reinforced polymer composite material incorporating hierarchical mutually off-set, but highly aligned fibre reinforcement with integration and directional control across nano, micro and meso length scales. High performance structural reinforcement targeted for additive layer manufacturing offers the potential for 'building' multifunctional composite materials in both controlled manufacturing environments and at the front line ensuring the timely introduction of new designs in a rapidly changing theatre of conflict.

\section{References}

[1] P. Fratzl, Cellulose and collagen: from fibres to tissues, Current Opinion in Colloid \& Interface Science 8 (2003) 32 - 39.

[2] P.Fratzl, R. Weinkamer, Nature's hierarchical materials, Progress in Materials Science 52 (2007) $1263-1334$. 
[3] G. Otieno, A. Koos, F. Dillon, A. Wallwork, N. Grobert, R. Todd, Processing and properties of aligned multi-walled carbon nanotube/aluminoborosilicate glass composites made by solgel pro- cessing, Carbon 48 (2010) $2212-2217$.

[4] W. Wang, P. Ciselli, E. Kuznetsov, T. Peijs, A. Bar- ber, Effective reinforcement in carbon nanotubepolymer composites, Philosophical transactions. Series A, Mathematical, physical, and engineering sciences 366 (2008) $1613-1626$.

[5] L. Jin, C. Bower, O. Zhou, Alignment of carbon nanotubes in a polymer matrix by mechanical stretching, Applied Physics Letters 73 (1998) 1197 - 1199. 


\section{Research Methodology}

The work undertaken within this 12-month Seed Project study is primarily experimental with some supporting numerical components. The specific scientific objectives set out at the onset of the research project are captured below:

[1] To control reinforcement alignment and fibre staggered spacing by ultrasonic manipulation.

[2] To select and adapt a host polymer matrix (e.g. polyester, vinylester or epoxy) to make it suitable for suspension and 'fixing' of the aligned fibre configuration after ultrasonic manipulation.

[3] To maximise fibre volume fraction with hierarchical reinforcement

[4] To experimentally and analytically characterise the material produced in [2] and [3].

To address these objectives three parallel investigations were undertaken, namely:

a) Development of hierarchical fibres (for ultrasonic alignment)

b) Ultrasonic alignment of nanowhiskers reinforcement

c) Ultrasonic alignment of micron length scale reinforcement

The results of these investigations are reported in the following sections. 


\section{Ultrasonic alignment of nanowhiskers reinforcement}

\subsection{Introduction}

The use of acoustic radiation forces to manipulate particles has proven useful across a number of different disciplines, most notably within microfluidics for biomedical purposes [1,2] (e.g cell manipulation and separation), using standing waves with trap locations determined by the dimensions of the system. There has been much less research into the use of such acoustic radiation pressure to manipulate and orient cylindrical particles (i.e fibres), particularly from an experimental perspective. The manipulation and alignment of short fibres with an ultrasonic field could provide a solution to the problem of manufacturing unidirectional short fibre composite materials. Cellulose is an interesting material for composite manufacturing, as it is abundant and cheap, and an environmentally friendly alternative to synthetic fibres. The use of cellulose nanowhiskers could produce composites with improved mechanical properties due to the high tensile strength of the whiskers, which would remove one of the largest issues with current cellulose reinforced composites. Due to the small size of the whiskers, however, conventional techniques for producing unidirectional reinforcements are ineffective. Previous work has shown the efficacy of using strong magnetic fields to align cellulose nanowhiskers[3] (CNW's) for the purpose of producing nanocomposite [4]. It has been shown that 3-dimensional and layered structures can be formed using magnetic field alignment of particles coated with superparamagnetic nanoparticles, but in order to optimise the system for minimal magnetic field strength the size and shape of the particles are constrained [5]. Acoustic trapping can provide solutions to situations where other trapping techniques are unsuitable, as is commonly the case with the manipulation of cells with optical tweezers, as the laser intensity required for trapping can cause damage to the cells [6]. Acoustic trapping also has the advantage of being able to manipulate large numbers of particles simultaneously with relative ease, making this an ideal technique for particle filtering.

Much of the previous work with sonotweezers has revolved around the principle of manipulating particles to desired positions by varying the position of the trap, which has successfully been achieved by producing standing wave from two 
counter-propagating waves and varying the phase between the transducers [7]. In this work, the aim is to produce a patterned distribution of nanowhiskers with a common orientation, with no need for further manipulation, thus allowing the acoustic field to remain unchanged throughout the process. The most important factor then becomes the use of an appropriate frequency to maximise the forces on the nanowhiskers.

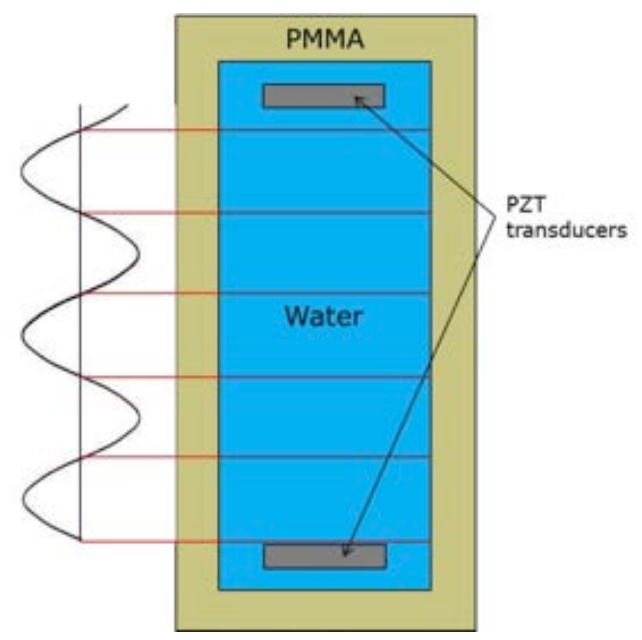

Figure 3.1: Schematic representing the transducers producing a $2 \mathrm{MHz}$ standing wave field with counter propogating waves. The dashed lines show the pressure nodes where dense particles are trapped

A schematic of the initial rig design is shown in Figure 3.1.The transducers are lead zirconate titanate (PZT) piezoceramic plates, and are generally used at their lowest frequency mode of $2 \mathrm{MHz}$. The dimensions of the water filled cavity are $80 \mathrm{~mm}$ $x 30 \mathrm{~mm}$, which is slightly larger than a microscope slide so that samples can be produced on a slide and removed. Within Figure 3.1, the horizontal lines represent the nodal pressure positions where dense particles are trapped.

The frequencies used in this work are unlikely to align individual nanowhiskers, and therefore no surfactant was used to produce a stable dispersion of separate whiskers. Instead, it is hoped that by manipulating clumps of nanowhiskers into traps, the concentrations of nanowhiskers within the confined regions would be large enough to form a nematic phase, thus creating the desired alignment [8]. The local concentrations required to form such a liquid crystal, however, are very high, and it is not known whether the acoustic radiation forces on the fibres will be sufficient for long range rotational order to become energetically 
favourable. It is hoped that the fibres may partially align due to the acoustic pressure gradient prior to confinement within the traps. There has been some investigation into the alignment and trapping processes separately, and a semi-empirical model has been shown to describe the associated velocities of these processes [9]. The equaltion is given below:

$$
\begin{aligned}
& v(x) \propto-r \sin [2 k x] \\
& \omega(\theta) \propto-\left(r / l^{2}\right) \sin [2 \theta]
\end{aligned}
$$

Here ' $v$ ' is the velocity of the centre of mass of the fibre towards a trap position and ' $\omega$ ' is the angular velocity of the fibre towards an aligned position, ' $x$ ', ' $r$ ' and ' $\mathrm{l}$ ' are the position, radius and length of the fibre respectively, and ' $\theta$ ' is the angle of the fibre away from the aligned orientation. As the angular velocity of the fibre is dependent upon the radius over the length squared, the alignment process will occur more slowly for longer fibres and fibres with a large aspect ratio.

Previous work has been performed within ACCIS to align glass microfibres with acoustic radiation pressure [10]. Within this research, epoxy-glass composite materials with ultrasonically aligned fibres have been produced in thin panels, and the resulting material exhibit anisotropic in-plane mechanical properties. To date, the aim has been to produce unidirectional composite materials with a simple acoustic field produced by opposing flat transducers. Theoretically, acoustic radiation pressure could enable more complex 3-dimensional arrangements of the reinforcing fibres to be achieved.

The initial theoretical work to determine the force on particles due to acoustic radiation pressure is accredited to King [11]. A number of assumptions were made in this initial work, as the force was determined for incompressible spherical particles in an inviscid medium. More recently, extensions to this work have been made which take into account the compressibility of the spheres and the viscosity of 
the medium $[12,13]$, and some which determine the force on a cylindrical particle [14].

The acoustic pressure on spherical particles causes a force which can be considered as simply acting about the centre of mass, resulting in a translational displacement of the particle and ignoring any change in angular momentum. In the case of a cylindrical particle, however, the variation in the local pressure along the length of the particle can result in a rotational torque as well as this translational force [9]. It is clear that the dimensions of the particle determine the optimal frequency for manipulation. It has been shown that the length of the fibre must be less than a quarter of a wavelength [16], which is an obvious constraint to prevent the fibre 'bridging' across neighbouring pressure nodes and thus experiencing no net aligning force. However, in the case that the length of the fibre is many orders of magnitude smaller than the wavelength of the acoustic field, the pressure difference across the length of the fibre will be small due to the low pressure gradient. This will result in small aligning torques, which may not be sufficient to even partially align the fibres. It is crucial, therefore, to optimise the wavelength of the field for the given fibre sizes. Problems will occur when the length of the fibres is not uniform, especially in the case of unusually large fibres, as small fibres may not be aligned but are generally confined within a trap. Large fibres are not trapped, however, and intermolecular forces mean that smaller fibres collect around a large fibre, preventing their alignment.

\subsection{Pressure field monitoring}

A model was produced which mapped the predicted acoustic field pressure amplitude between two transducers placed at a given distance from one another. With the transducers perfectly aligned and parallel to each other, vibrating at the same frequency and amplitude, a simple standing wave pattern was produced, as shown in Figure. In order to determine the resulting acoustic field, a finely spaced Cartesian grid was created such that there were at least 10 grid points per wavelength to provide a high resolution for plotting purposes. This grid was used as the area over which the pressure amplitude was calculated and finally plotted. The transducers were defined by lines between two points, also in Cartesian coordinates, 
which were then split into individual Huygens sources, ensuring that the spacing between each point source was less than or equal to half a wavelength to prevent the formation of grating lobes. The transducers were defined outside of the grid area to avoid singularities caused by zero distance values. The total distance from each transducer point source to each point in the grid was then calculated, and the acoustic pressure amplitude was calculated using the following expression:

$$
p_{i}=\frac{e^{i k r}}{\sqrt{r}}
$$

Here, ' $r$ ' is the total distance of the grid point from the transducer source point, ' $\mathrm{k}$ ' is the wavenumber and ' $\mathrm{p}_{\mathrm{i}}$ ' is the pressure resulting from a single Huygens source point. The result of this is essentially a circular wavefield pattern from each point source, from which the total pressure amplitude, ' $p_{\text {total }}$ ', produced by the transducers is computed through superposition by summing over all of the source points and taking the absolute value of this complex result:

$$
p_{\text {total }}=\left|\sum_{i=1}^{N} p_{i}\right|
$$

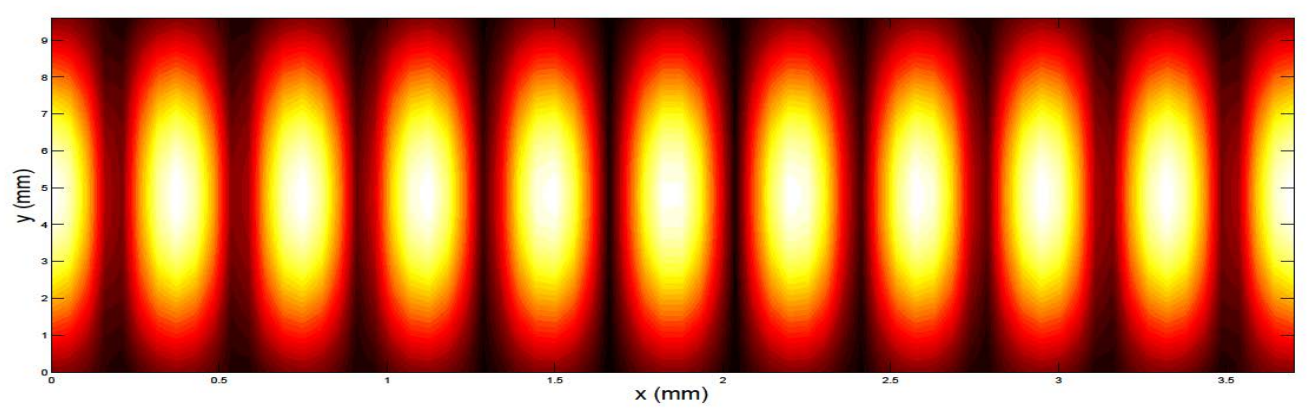

Figure 3.2: Acoustic pressure amplitude at the centre point between two perfectly parallel transducers separated by $80 \mathrm{~mm}$. Both transducers are operating at a frequency of $2 \mathrm{MHz}$. The plotted region corresponds to 5 wavelengths in the $x$-direction by 13 wavelengths in the $y$-direction

According to the theory discussed previously the use of high frequencies is essential to manipulate individual CNW's [9], as with low frequencies the pressure gradient will not be sufficient to align nanoscale fibres. As a result of this, any 
misalignment of the transducers becomes increasingly critical, as this causes a greater phase change of the wavefronts along the length of the transducer. The effects of this are shown in Figure 3.3. At a frequency of $2 \mathrm{MHz}$, the shape and position of the acoustic traps with both transducers rotated anticlockwise by $2^{\circ}$ are very similar to those for perfectly aligned transducers (Figure 3.2). However, for the same $2^{\circ}$ at $6.8 \mathrm{MHz}$, which roughly corresponds to the second resonant mode of the transducers used, the shape of the traps has been significantly distorted. The maximum pressure of the system is reduced by approximately $30 \%$ for a $2^{\circ}$ rotation at $6 \mathrm{MHz}$, while the same rotation at $2 \mathrm{MHz}$ only results in a $10 \%$ reduction in the maximum pressure of the system. The minimum pressure amplitude of the system is increased as a result of the rotation of the transducers, with the increase in the $6.8 \mathrm{MHz}$ field being 3 times greater than in the $2 \mathrm{MHz}$ field. As the periodicity of the wavefields remains the same, both of these factors result in a smaller acoustic pressure gradient, which is more significantly affected with increasing frequency.
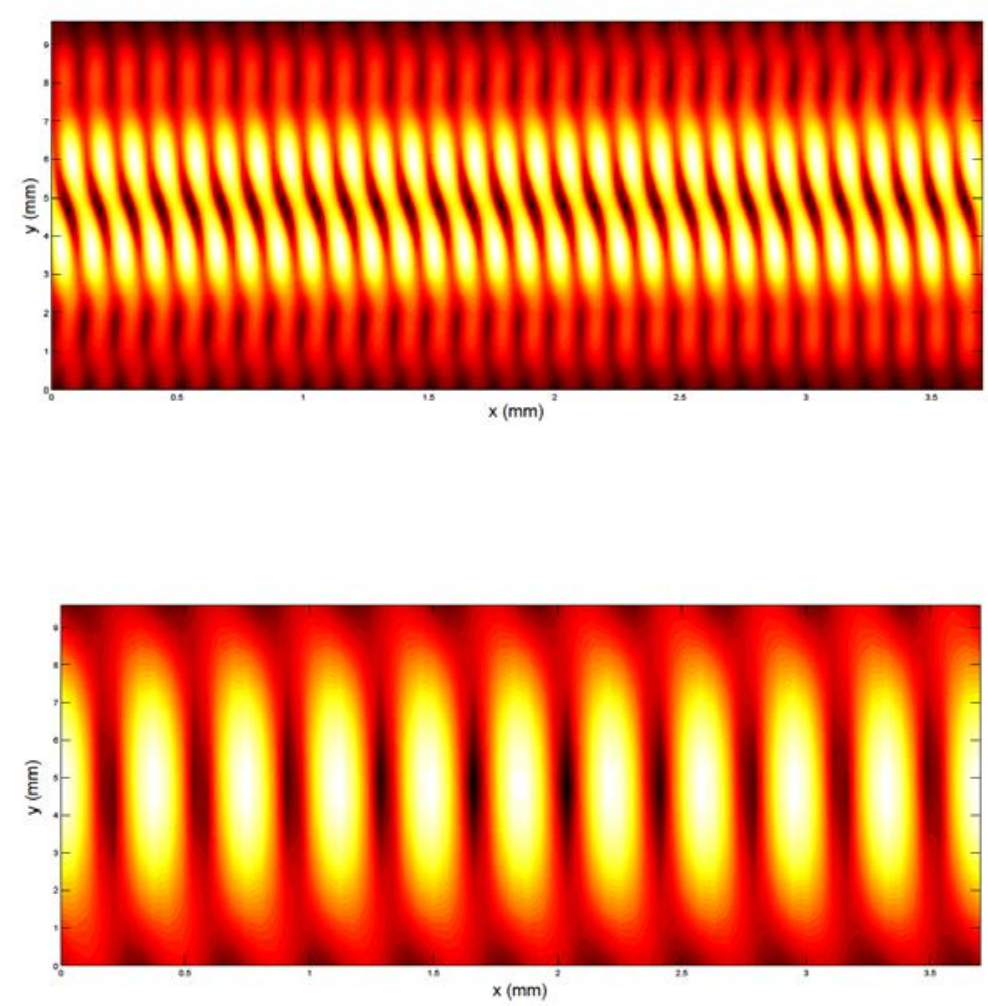

Figure 3.3: Acoustic pressure field for the same transducers as in Figure 3.2 over the same spatial area, with both transducers rotated by 2 degrees anticlockwise. The operating frequencies are $6 \mathrm{MHz}$ (top) and $2 \mathrm{MHz}$ (bottom) 


\subsection{Experimental Validation}

As aforementioned, misalignment of the transducers can lead to significantly reduced acoustic radiation forces. To improve the transducer alignment, a rig was produced which allowed for the transducers to be rotated, as shown in Figure 3.4. This also enabled a secondary experiment to be performed, wherein the transducers were deliberately rotated through a certain angle, and the locations of the traps were determined by tracking the position of polystyrene microparticles in a solution within the rig. The results of this were then compared with the expected acoustic field predicted by the model.

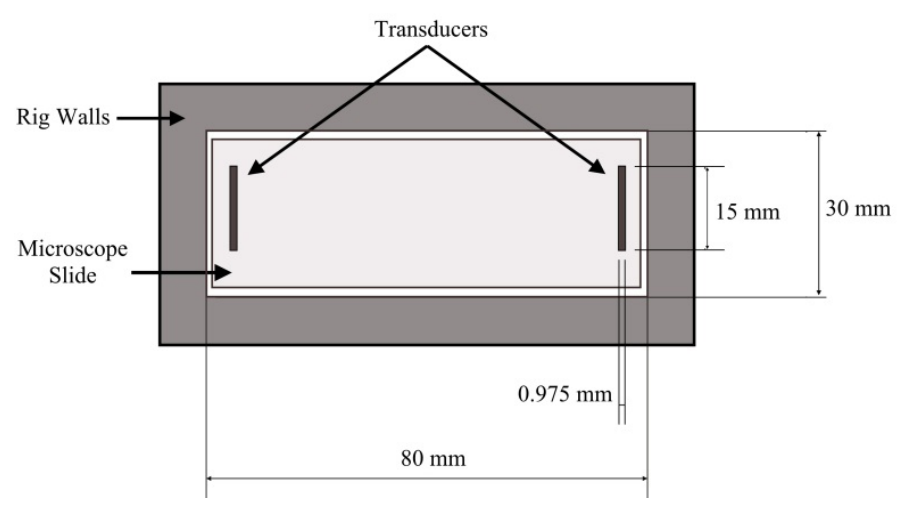

Figure 3.4: Rig design allowing for rotation of transducers

To perform the initial calibration to ensure the transducers were as ideally aligned as possible, one of the transducers was connected to a signal generator, and the other to an oscilloscope. The transducers were initially aligned by eye, and the signal generator was then used to drive one transducer at its lowest resonant mode, roughly $2 \mathrm{MHz}$, at the maximum possible voltage, $10 \mathrm{~V}_{\mathrm{pp}}$ (Volts peak-to-peak). Higher voltages are possible with the use of an amplifier but generally lead to acoustic streaming. By rotating the transducers slowly, the oscilloscope was used to determine the point at which the signal response had maximum amplitude, which corresponds with parallel alignment of the transducers

In order to verify the model, a solution of $1 \mu \mathrm{m}$ diameter polystyrene spheres was placed in the rig. These particles are easily trapped by the acoustic radiation pressure, and with suitable concentrations they provide a clear indication of the locations of the traps with a given acoustic field. $200 \mu \mathrm{l}$ of $10 \%$ polystyrene microsphere solution was mixed with $4 \mathrm{ml}$ of water and placed within the rig cavity. The transducers were calibrated to be aligned and driven with $30 \mathrm{~V}_{\mathrm{pp}}$ at a frequency $2 \mathrm{MHz}$ until the trap sites were clearly visible by the 
locations of the microspheres, which took roughly 1 minute. Following, the transducers were rotated by $2^{\circ}$ anticlockwise and then driven at the same frequency and voltage to compare the effects of misalignment with those predicted by the model. The results of this are shown in Figure 3.5.
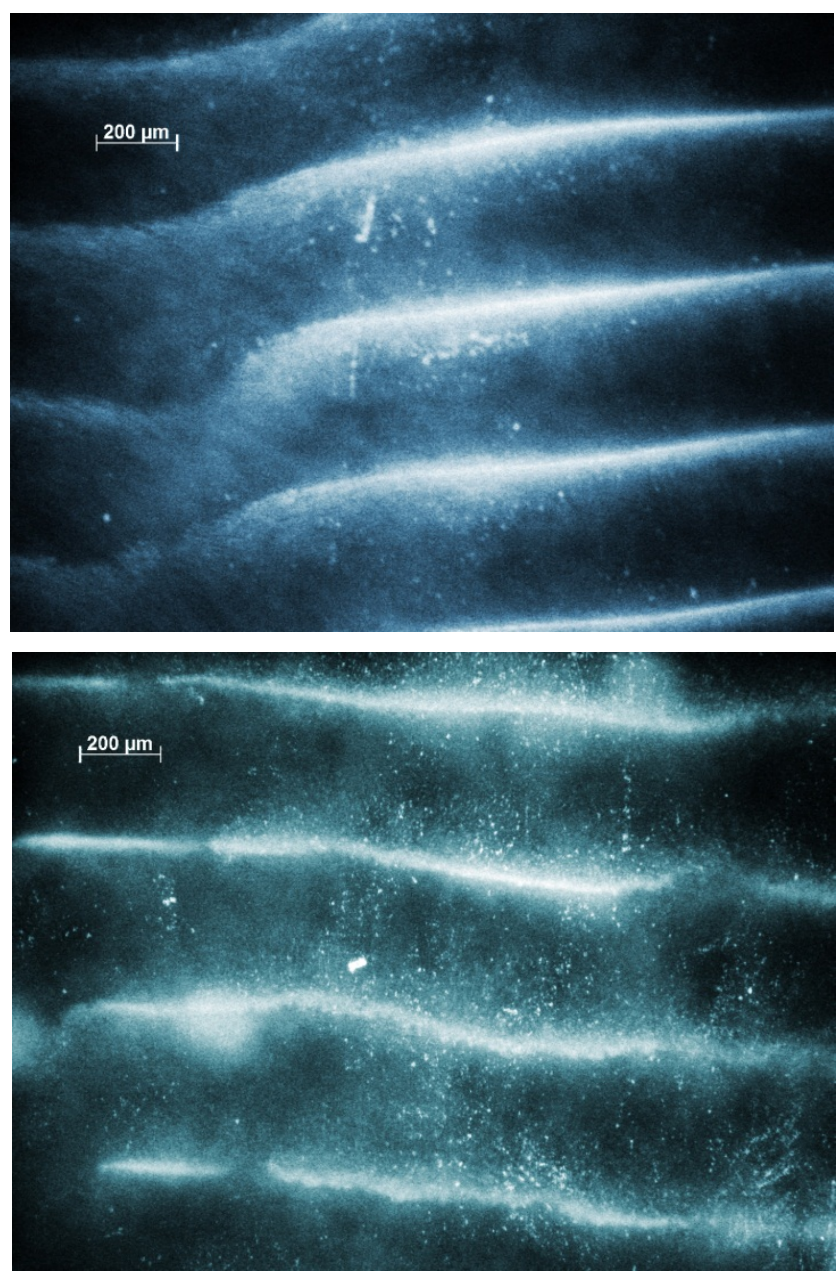

Figure 3.5: 1 m polystyrene microspheres trapped in a $2 \mathrm{MHz}$ acoustic field (a) with aligned transducers $(b)$ with transducers rotated by $2^{\circ}$

It can be seen in Figure 3.5 that with aligned transducers, the trapping areas are reasonably uniform straight lines, with some waviness. With a $2^{\circ}$ rotation of the transducers however, the traps are also slightly rotated, and the edges of the traps are significantly distorted, as expected in Figure 4. Overall, however, the traps remain distinct and the shape is not globally affected. The same procedure was performed with a frequency of $6.77 \mathrm{MHz}$ and a driving voltage of $4 \mathrm{Vpp}$, and the results are shown in Figure 3.6. 

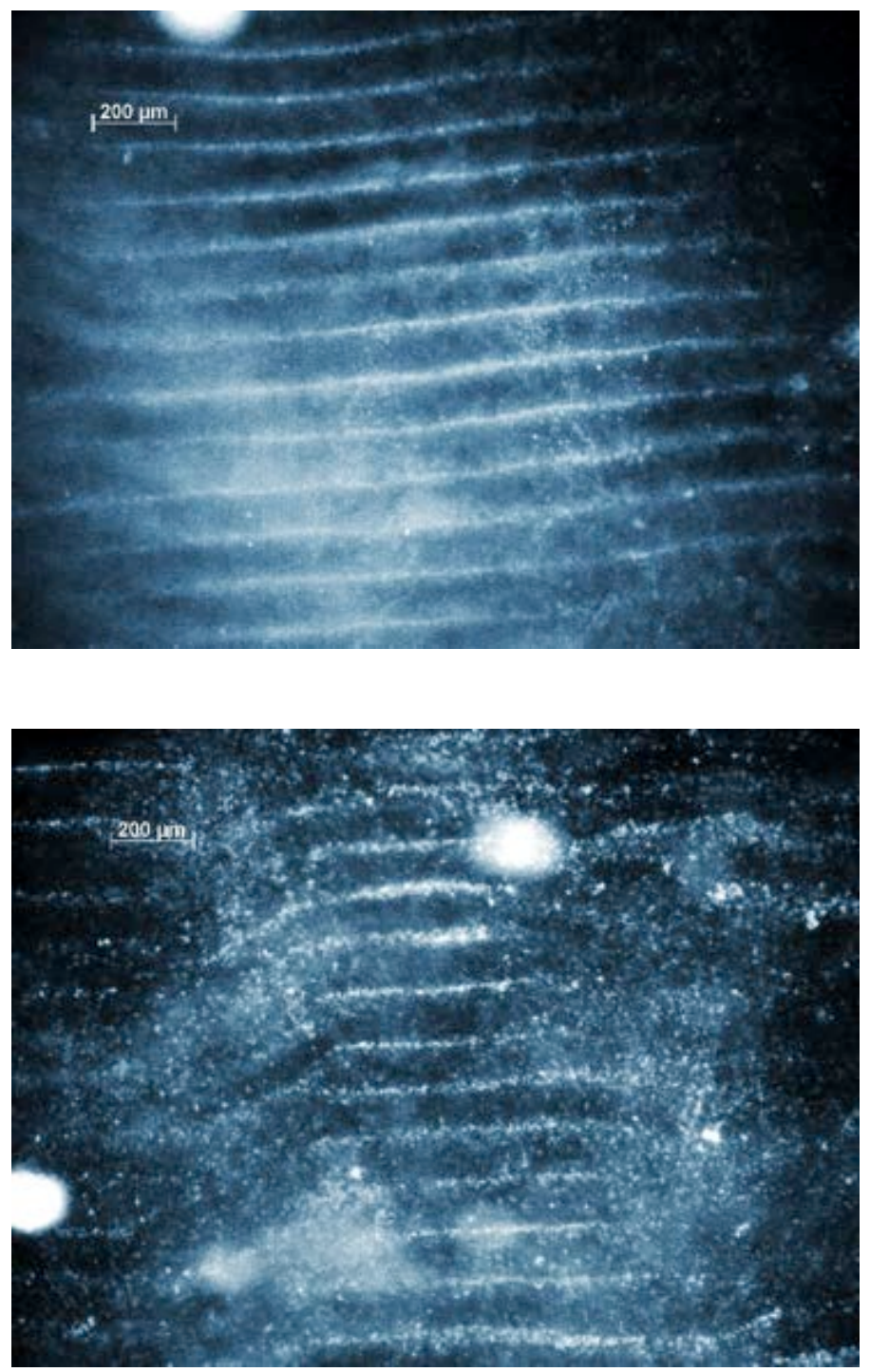

Figure 3.6: $1 \mu \mathrm{m}$ polystyrene microspheres trapped in a $6 \mathrm{MHz}$ acoustic field (a) with aligned transducers (b) with transducers rotated by $2^{\circ}$

The driving voltage used at $6.77 \mathrm{MHz}$ was significantly lower than with $2 \mathrm{MHz}$ to minimise acoustic streaming whilst still applying sufficient forces to the microspheres for manipulation. As a result the particles took roughly 5 minutes to move to the pressure nodes. With aligned transducers, the traps form straight and parallel lines, as predicted by the model. With transducers rotated by $2^{\circ}$, the effective trapping area was dramatically reduced, with only a very narrow band forming an effective trapping site. This agrees well with what is predicted by the model. 


\subsection{Experimental Validation}

\subsubsection{Cellulose Nanowhisker Alignment}

The initial calibration procedure was performed to align the transducers. To determine whether the traps were clearly defined parallel lines as expected, a glass microfibre solution was placed within the rig, and their alignment within the pressure traps was checked. Once this was completed, the solution was removed and the rig cleaned in preparation for using a CNW solution.

The cellulose nanowhiskers used were provided by Professor Stephen Eichhorn from the CEMPS Department at Exeter University. The nanowhiskers supplied are in a dry form, and in this state they are in large clumps. Two different samples were made, wherein these clumps were broken down to varying degrees. Whilst the specific batch of nanowhiskers used in this project have not been characterised, similar nanowhiskers produced with the same method by the same group have been [8]. These nanowhiskers are derived from cotton, and have dimensions of around $130 \mathrm{~nm} \times 20 \mathrm{~nm}$, giving them a relatively small aspect ratio of around 6. To determine whether individual nanowhiskers (or at least very small clumps) could be manipulated, a solution of the nanowhiskers was produced by mixing the nanowhiskers in water and then sonicating for 60 minutes. The second sample contained larger clumps of the nanowhiskers, and was produced by mixing the nanowhiskers in water and stirring to break down the larger clumps. In both cases, only fibres from near the surface of the solution were used to produce the sample to avoid using excessively large clumps of the nanowhiskers which settled at the bottom of the solution.

A microscope slide was placed on the floor of the rig cavity, so that dried samples of aligned nanowhiskers could be removed and the rig reused. The rig was filled with the nanowhiskers solution such that the transducers were just fully submerged. The signal generator was then used to apply a standing wave field with a frequency of $2 \mathrm{MHz}$ (giving a wavelength of $736 \mu \mathrm{m}$ ) using a driving voltage of 10Vpp .At this stage the alignment process occurs, with the nanowhiskers sitting at the pressure nodes of the acoustic field. A needle syringe is then used to remove water 
slowly from the corner of the rig to reduce drying time. The acoustic pressure field is applied for 30 minutes, after which point the signal generator is switched off. The sample is then left undisturbed to dry out over a period of 24 hours, at which point a dry sample of lines of nanowhiskers is left on the microscope slide in the bottom of the rig, which can then be removed and analysed.

To determine whether any alignment of the nanowhiskers was present, Polarised Raman Spectroscopy was used. Monochromatic, polarised laser light is focused on a small area of the sample ( 2 micron diameter). This light then excites vibrational modes within the system into virtual energy states. When the vibrational modes then relax to a lower energy mode, they emit a photon with the same energy as the energy difference between the initial excited mode and the relaxed state. Certain vibrational modes are polar, such that the polarisation of the incident laser light must align with the relevant vibration of the molecular structure in order to cause an excitation. Such an example exists within the cellulose nanowhiskers used in this case. Along the cellulose backbone, vibration of the $\mathrm{C}-\mathrm{O}-\mathrm{C}$ bond results in a peak at a Raman shift of $1095 \mathrm{~cm}-1$, and the intensity of this peak is greatly increased when the polarisation of the incident light is along the length of the cellulose chain. In the case of cellulose nanowhiskers, this corresponds to the long axis of the whisker. Therefore, if there is overall alignment of the nanowhiskers, the intensity of this peak should be highest when along the direction of alignment. In order to determine whether there was any alignment, Raman spectra were taken of a point along one of the 'stripes' of nanowhiskers of a dried sample while the laser polarisation was rotated, with one spectrum being taken every $3^{\circ}$ from $0^{\circ}$ to $90^{\circ}$. The spectra were then fitted to one another by removing signal noise (as a result of fluorescence) and correcting for variations in the incident laser intensity, in order to determine the real variation in the intensity of the peak at $1095 \mathrm{~cm}-1$ and therefore the degree of alignment of the sample. A second characteristic peak of cellulose occurs at a Raman shift of around $2900 \mathrm{~cm}-1$, which results from the vibration of the $\mathrm{C}-\mathrm{H}$ bonds within cellulose. These vibrations are non-polar, such that the polarisation of incident laser light has a negligible effect on the intensity of the Raman peak. By taking the ratio of the intensity of the peaks at $1095 \mathrm{~cm}-1$ and $2900 \mathrm{~cm}-1$, this gives a 
value proportional to the fraction of interacting cellulose that is aligned with the polarisation of the laser. This significantly simplifies the spectral fitting procedure, and reduces errors resulting from any variation in laser intensity due to rotation of the polariser.

\subsubsection{Frequency Modulation}

One of the primary issues with aligning fibres within an acoustic pressure field occurs as a result of the short timescale over which the alignment process occurs, particularly in a low viscosity medium such as water. As a result of this, the fibres move swiftly into the traps, with the frictional forces between fibres tending to resist the alignment process, resulting in randomly aligned clumps of nanowhiskers confined within the traps. In order to counter this, a method was devised to repeatedly 'relax' the fibres away from the trap and then force them back to the traps. To achieve this, the frequency of the transducers was modulated between $2 \mathrm{MHz}$ and $6 \mathrm{MHz}$. The lower frequency mode produced a larger force on the fibres, and so they were gradually confined within the trap sites produced by this frequency.

\subsection{Experimental Results and Discussion}

\subsubsection{CNW Alignment}

The results of the initial calibration using glass fibres shows that the traps are well defined and align fibres on this length scale effectively.

Two samples were analysed using the Raman spectroscopy technique described previously. Both samples were produced with a $2 \mathrm{MHz}$ applied acoustic field, and allowed to dry at room temperature. An example of the CNW solution with the acoustic field applied is shown in Figure 3.7. 


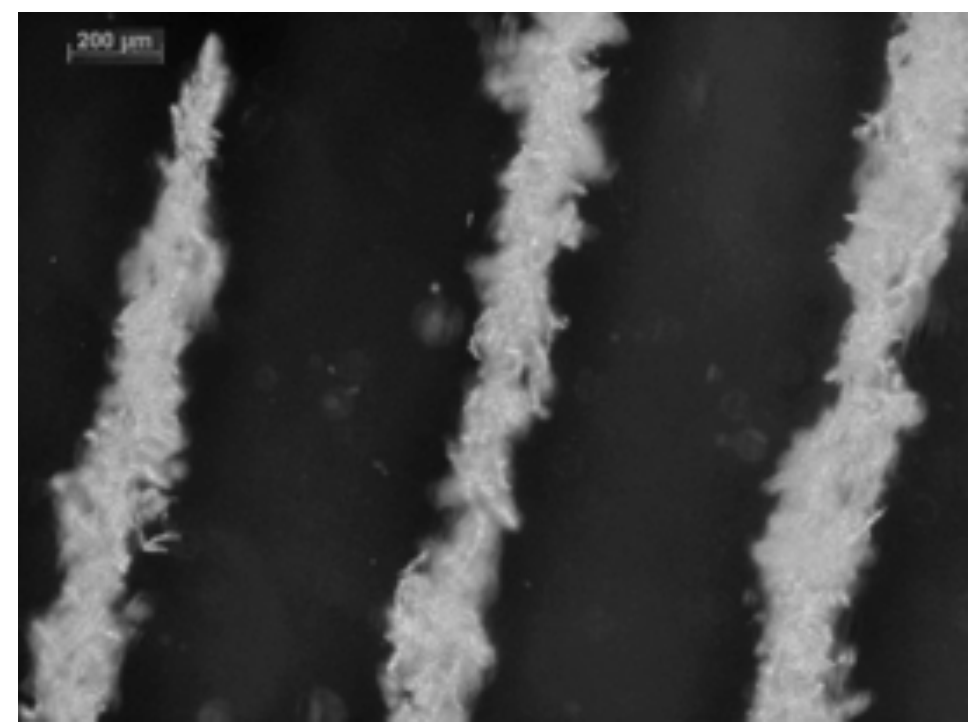

Figure 7: Optical Microscopy (Zeiss Axio Imager M2, 5x objective) image of CNW's with $2 \mathrm{MHz}$ standing wave field applied

The spectra need correction for varying levels of noise (resulting from fluorescence) before the degree of alignment of the fibres can be determined. A baseline correction for the spectra was performed. It was then assumed that the intensity of the peak at $2900 \mathrm{~cm}^{-1}$ was invariant, which is a reasonable assumption if the region of the sample analysed does not vary [17]. Having scaled the spectra accordingly to normalise against this peak, the variation in the intensity of the peak at $1095 \mathrm{~cm}^{-1}$ could be determined. The corrected and normalised spectra for each sample are shown in Figure 3.8. The peaks visible in sample 1 at around $3100 \mathrm{~cm}^{-1}$ are the result of high energy cosmic rays incident on the CCD array. 

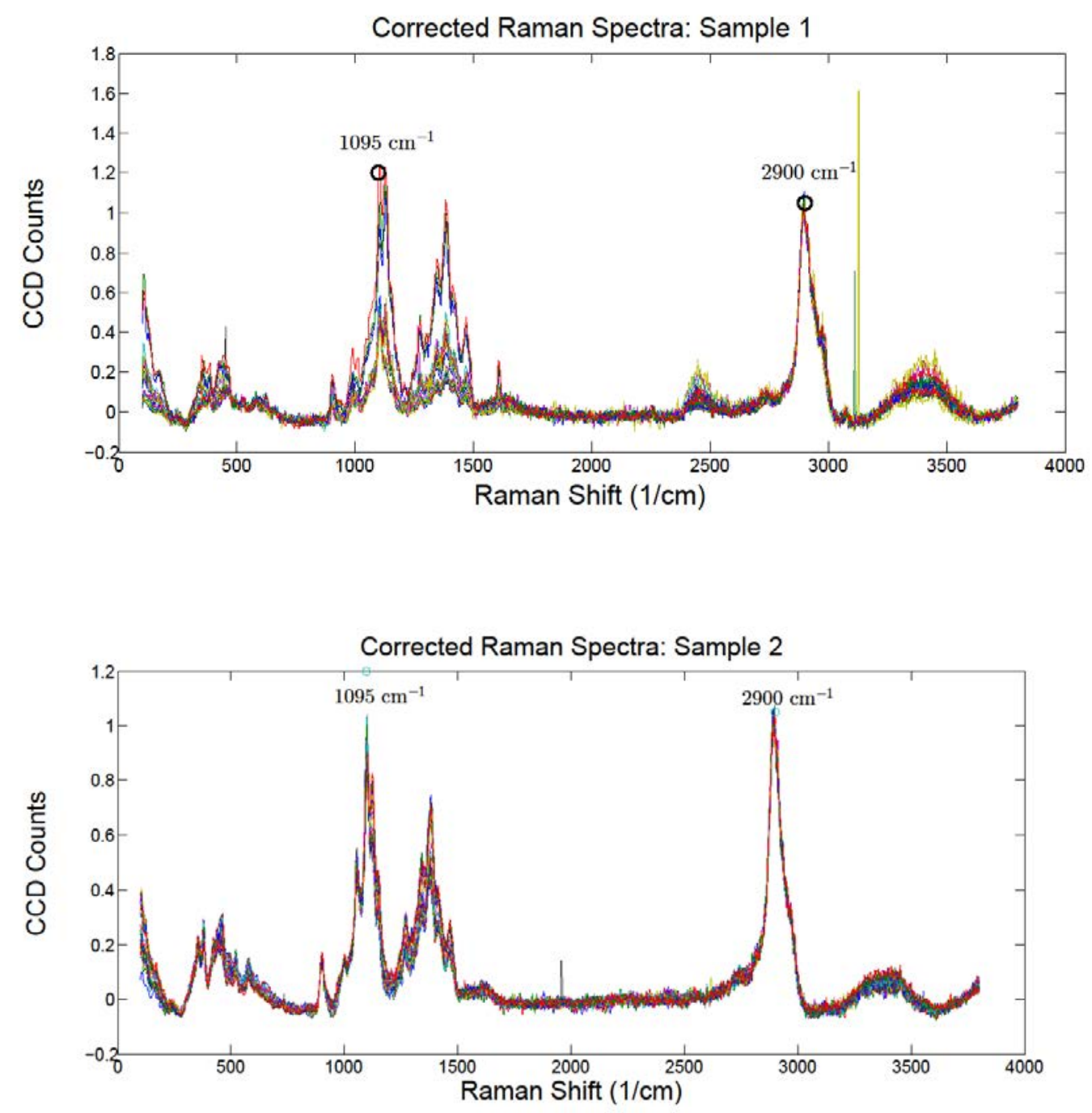

Figure 3.8: Corrected, normalised Raman spectra of the two dried samples of aligned CNW's

To clearly visualise the variation of the intensity of the peak with angle, a polar plot is used, with the angle between the light polarisation and the axis of the acoustic traps represented by $\theta$ and the intensity of the peak for that spectra represented by ' $r$ '. The results are shown in Figure 3.9. As the distance of each point from the origin represents the ratio between the intensity of two Raman shift peaks, the value is dimensionless, and only the relative value of different points at different angles can be used. This gives a qualitative idea of the degree of alignment of the nanowhiskers in the sample, with a perfectly circular plot showing entirely random orientation of the local particles and a thin flat line representing perfect alignment in 
a single direction. As spectra were only taken from $0^{\circ}$ to $90^{\circ}$, these values were then mirrored to form a full polar plot which gives a clearer impression of the anisotropy of the sample.

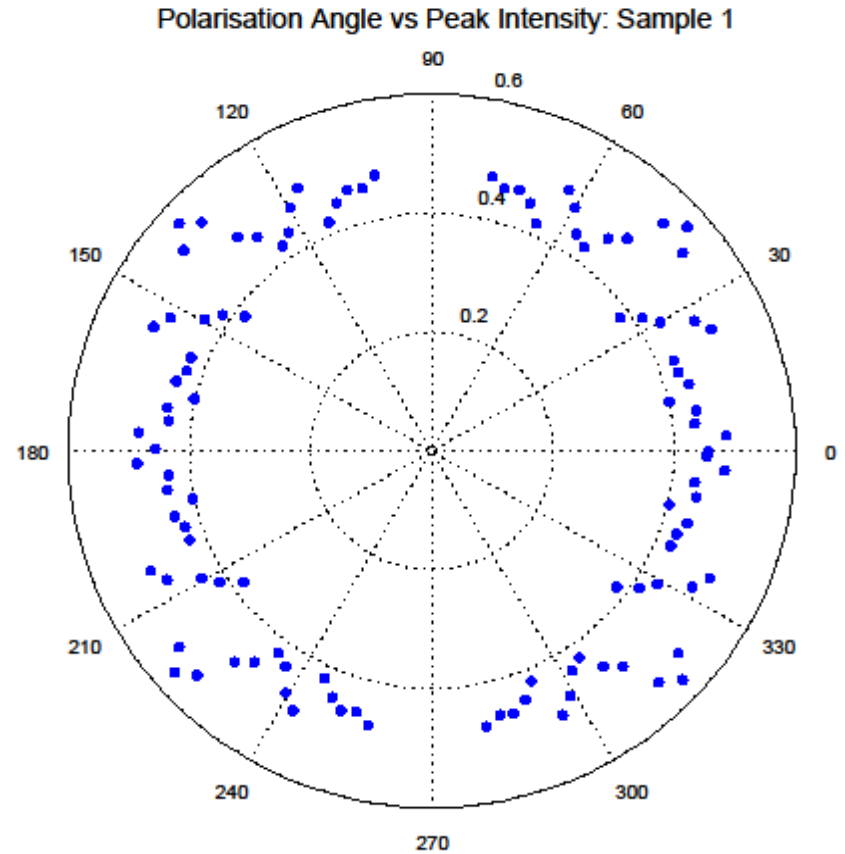

Polarisation Angle vs Peak Intensity: Sample 2

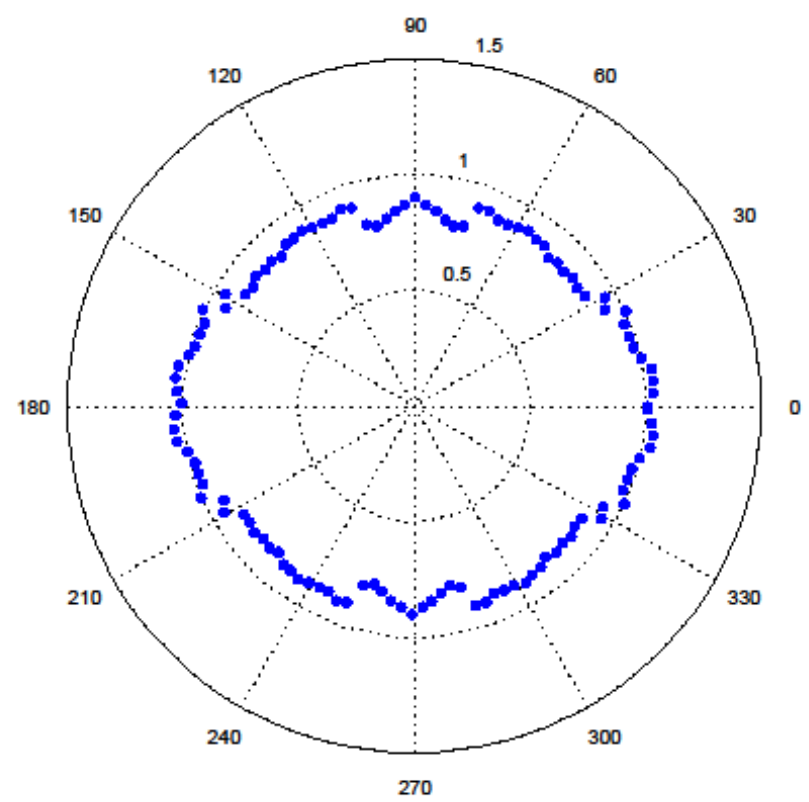

Figure 3.9: Variation in $1095 \mathrm{~cm}^{-1}$ peak intensity with polarisation angle.

The polar plots for the two samples give contrasting ideas about the possible alignment of the nanowhiskers within the sample. Sample 2 appears to show some

Distribution A: Approved for public release; distribution is unlimited. 
anisotropy, with a preferred orientation along the length of the acoustic traps, although the maximum variation in intensity with angle is small (approximately 4/3). The results of sample 1 appear to show a more uniform intensity with angle, and therefore a lack of any alignment within the sample. The spectra from sample 1 generally had higher levels of background noise, making analysis of the spectral peaks more difficult and more prone to error, but overall there is no strong indication of alignment of individual nanowhiskers within either sample. The spectra for sample 1 with polarisation angles from $81^{\circ}$ to $90^{\circ}$ had exceptionally high fluorescence levels, which made it impossible to accurately determine the intensity of any peaks present, and so this data has been omitted. Possible explanations for the anisotropy observed in sample 2 are:

- Acoustic radiation pressure creating high concentration regions of CNW's, which self-assemble into a nematic phase.

- Acoustic manipulation of locally aligned clumps of CNW's into trap sites.

- Coincidental local alignment of sampled region.

It is unlikely that the observed anisotropy is a result of coincidental local alignment of the whiskers, as the sampling region will have contained thousands of whiskers. Due to the relatively low forces created by the acoustic radiation pressure, it is suggested that the observed anisotropy is a result of the manipulation of internally self-oriented clumps of CNW's. A representation of this distribution is shown in Figure 3.10 . 


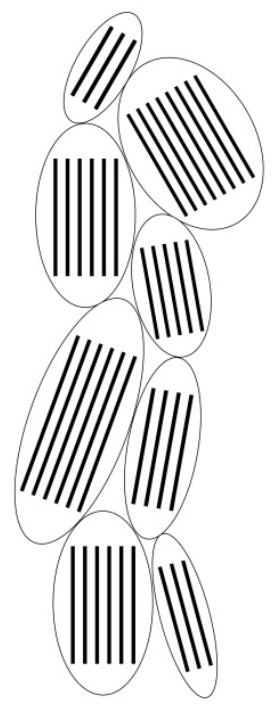

Figure 10: Possible arrangement of clumps of aligned nanowhiskers which are manipulated as a group by the acoustic radiation pressure.

\subsection{Frequency Modulation}

The effect of switching between two separate resonant modes was made extremely clear when using the glass microfibres. Using a low modulation frequency, it was possible to observe the movement of the particles in real time between the adjacent traps. A secondary effect of this method meant that traps with a high fibre concentration 'leaked' fibres to neighbouring traps, producing a more uniform distribution of fibres in each band, as shown in Figure 3.11.
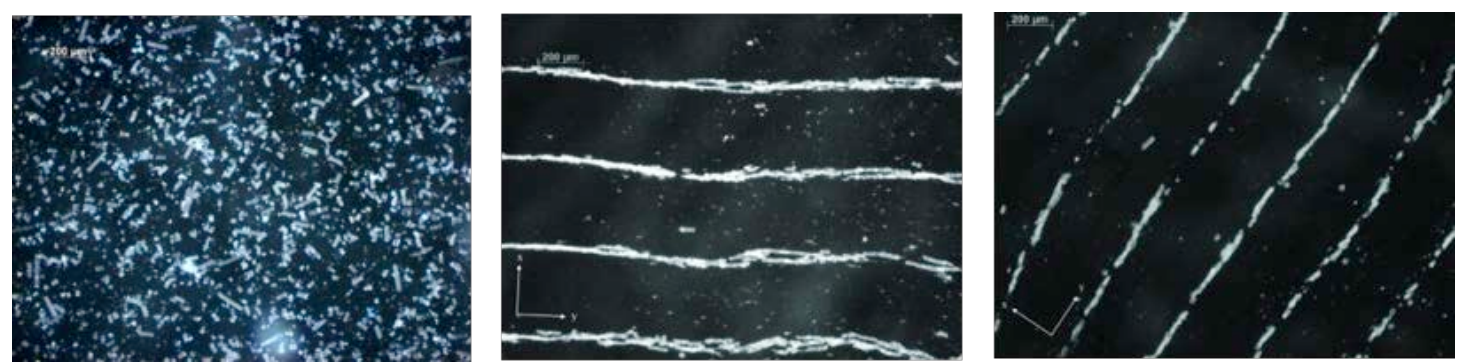

Figure 3.11: Optical Microscopy image of glass microfibre solution; (a) Randomly oriented with no acoustic field applied, (b) aligned with single $2 \mathrm{MHz}$ frequency, (c) aligned with frequency modulating between $2 \mathrm{MHz}$ and $6 \mathrm{MHz}$ at a frequency of $10 \mathrm{~Hz}$

There was less obvious improvement in the case of the CNW's when using this frequency modulation, which is potentially a result of the nanowhiskers propensity to agglomerate into clumps in solution, resulting in intermolecular forces 
which are greater than the separating forces applied by the modulating radiation pressure. Despite this, it is clear that for larger fibres with weaker intermolecular forces this modification of the alignment process gives improved alignment, albeit at a slower rate than with a single frequency, and shall be investigated in greater detail during future work.

\subsection{Conclusions}

The results indicate that the use of acoustic radiation pressure is not effective in driving the formation of a fully nematic phase in CNW's, with only a small degree of orientational order. The rapid manipulation of particles into trap sites does not seem to allow for the whiskers to align initially, and the acoustic forces are then not sufficiently strong to produce the concentrations required to form a liquid crystal. It has been shown that agglomerates of nanowhiskers can be manipulated into a macroscopic structure with partial alignment, however, and with more precise equipment it is entirely possible that global orientational order of individual nanowhiskers may be achieved. As previously discussed, this would require the use of significantly higher frequencies than those implemented in this work, and so the effects of streaming may become increasingly important. The use of simple frequency modulation has improved the alignment possible, indicating that there is a still much work to be done to optimise the process, and potentially reduce the precision required in the apparatus to work at higher frequencies.

\subsection{Future Work}

Having demonstrated the ability to produce macroscopic structures of CNW's within a fluid, it would be desirable to produce a sample of these CNW's in a polymer matrix for tensile testing, in order to determine whether the reinforcement is anisotropic. While the Raman results indicated that there is only partial alignment of the nanowhiskers, the analysis was only performed on small areas of the samples. Though the samples would not be expected to exhibit highly anisotropic material properties, it is important to definitively prove this experimentally. 
The use of ultrasonic fields has proven to be an effective method for aligning fibres, assuming that the frequency used is appropriate for the fibre dimensions. The use of nanofibres introduces a large number of new issues, which are largely down to the imprecision of the rig production. In order to overcome these issues, it is practical to make use of existing manufacturing techniques which are inherently extremely precise. Lithographic techniques with nanoscale precision are routinely employed in the production of Surface Acoustic Wave (SAW) devices, by etching and then printing microscale or even nanoscale interdigital transducers onto a piezoelectric substrate (commonly Lithium Niobate). The spacing between two opposing digits corresponds to $\lambda / 2$ of the surface wave produced (the spacing between two digits on the same side is equal to $\lambda$ ), as represented in Figure 12 [18]. As a result of using lithographic techniques, SAW devices can be produced with extremely high $Q$ values (>80000) which vibrate at extremely high frequencies $(>1 \mathrm{GHz})$ [19]. It is a future goal to repeat the process for alignment presented in this report using a SAW device in order to determine the alignment possible with more precisely manufactured equipment. This would effectively be an optimisation stage, with the goal of creating an effective manufacturing process.

There were a number of reasons for using cellulose nanowhiskers as the reinforcement in this work. The use of short fibres in composite materials mirrors materials seen in nature, and short cellulose fibres are present in the wall of plant cells. This structure contains concentric ring layers, each of which have a different fibre orientation, much like the composite laminates used commercially. Little is known about the properties of these nanowhiskers, however, making it difficult to optimise the system (e.g. fluid medium density/viscosity, substrate material) to maximise the effect of the acoustic radiation pressure. The aim in future would be to use other nanomaterials which have more suitable acoustic properties. Carbon nanotubes are theoretically an excellent material for this process due to their density and rigidity, but it is generally difficult to produce a stable dispersion of individual nanotubes which would be required 


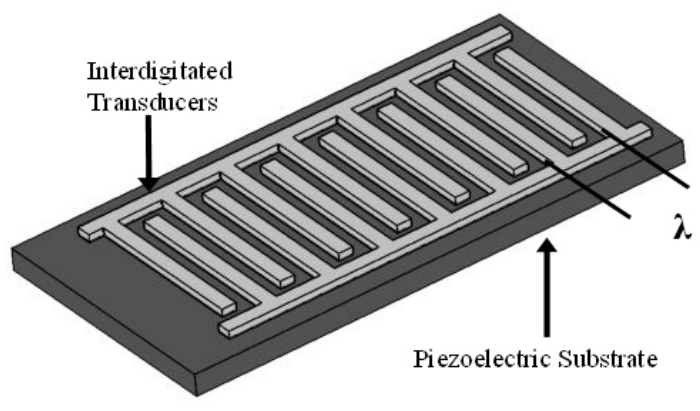

Figure 12: Overview of Surface Acoustic Wave device

In future work, there is to be a greater focus on the use of microfibres and also macrofibres, with the goal of extending this process into a continuous manufacturing process, either within existing Additive Layer methods or as an entirely independent process. The promising results from using magnetic fields to align particles in a plane $[4,5]$ indicate that using magnetic and acoustic fields in conjunction could lead to the ability to produce hierarchical structures with control of reinforcing particles on multiple length scales. Magnetic fields can be used to produce a unidirectional system of fibres, and the acoustic field used to produce a structure to the position of the fibres within the magnetically trapped plane. This may allow for nanofibres to be aligned, but optimisation of the concentration of paramagnetic nanoparticles would be required to allow for alignment with significantly altering the geometry and mechanical properties of the nanofibres themselves.

\subsection{References}

1 Mikael Evander, Linda Johansson, Tobias Lilliehorn, Jure Piskur, Magnus Lindvall, Stefan Johansson, Monica Almqvist, Thomas Laurell, and Johan Nilsson. Noninvasive acoustic cell trapping in a microfluidic perfusion system for online bioassays. Analytical chemistry, 79(7):2984-91, April 2007.

2 Jong Seob Jeong, Jung Woo Lee, Chang Yang Lee, Shia Yen Teh, Abraham Lee, and K Kirk Shung. Particle manipulation in a microfluidic channel using acoustic trap. Biomedical microdevices, 13(4):779-88, August 2011.

3 J. Sugiyama, H. Chanzy, and G. Maret. Orientation of cellulose microcrystals by strong magnetic fields. Macromolecules, 25(16):4232-4234, August 1992.

4 Tanittha Pullawan, Arthur N Wilkinson, and Stephen J Eichhorn. Influence of magnetic field alignment of cellulose whiskers on the mechanics of all-cellulose nanocomposites. Biomacromolecules, 13(8):2528-36, 2012 
5 Randall M Erb, Rafael Libanori, Nuria Rothfuchs, and Andre R Studart. Composites reinforced in three dimensions by using low magnetic fields. Science (New York, N.Y.), 335(6065):199-204, January 2012.

6 Hu Zhang and Kuo-Kang Liu. Optical tweezers for single cells. Journal of the Royal Society, Interface / the Royal Society, 5(24):671-90, July 2008.

7 Alon Grinenko, Paul D Wilcox, Charles R P Courtney, and Bruce W Drinkwater. Proof of principle study of ultrasonic particle manipulation by a circular array device. Proceedings. Mathematical, physical, and engineering sciences / the Royal Society, 468(2147):3571-3586, November 2012.

8 Stephen J. Eichhorn. Cellulose nanowhiskers: promising materials for advanced applications. Soft Matter, 7(2):303, January 2011.

9 P Brodeur, J L Dion, J J Garceau, G Pelletier, and D Massicotte. Fiber characterization in a stationary ultrasonic field. IEEE transactions on ultrasonics, ferroelectrics, and frequency control, 36(5):549-553, 1989.

10 M. Scholz. Rapid prototyping manufacture of structurally efficient short fibre composite materials, 2013.

11 L. V. King. On the Acoustic Radiation Pressure on Spheres. Proceedings of the Royal Society A: Mathematical, Physical and Engineering Sciences, 147(861):212-240, November 1934.

12 A A Doinikov. Acoustic Radiation Pressure on a Compressible Sphere in a ViscousFluid. Journal of Fluid Mechanics, 267:1-21, 1994.

13 A. A. Doinikov. Acoustic Radiation Pressure on a Rigid Sphere in a Viscous Fluid, 1994.

14 F.G. Mitri. Radiation force acting on an absorbing cylinder placed in an incident plane progressive acoustic field, 2005.

15 Takahi Hasegawa. Acoustic radiation force experienced by a solid cylinder in a plane progressive sound field. The Journal of the Acoustical Society of America, 83(5):1770, 1988.

16 P. Brodeur. Motion of fluid-suspended fibres in a standing wave field, 1991.

17 James H. Wiley and Rajai H. Atalla. Band assignments in the raman spectra of celluloses. Carbohydrate Research, 160:113-129, 1987.

18 R. M. White and F. W. Voltmer. Direct Piezo-electric coupling to surface elastic waves. Applied Physics Letters, 7(12):314, 1965.

19 C.K. Campbell. Applications of surface acoustic and shallow bulk acoustic wave devices. Proceedings of the IEEE, 77(10):1453-1484, 1989. 


\section{Development of hierarchical fibres (for ultrasonic alignment).}

\subsection{Introduction}

Structural hierarchy at the macro scale is generally well established, providing a structure that constitutes several length scales such as the Eiffel tower [1]. However, hierarchy has had limited use in man-made materials [2]; engineering materials such as metals and composites have hierarchy at a length scale much larger than observed in nature. Hierarchy in a material is sometimes conjectured to improve specific strength and stiffness [1], allowing relatively weak, more widely available constituents to be used to obtain high-performance macro-scale properties [3]. Biological materials such as bone are naturally occurring composites that exhibit a rich hierarchical structure $[1,4,5]$. Approximately seven levels of hierarchy are present: bone tissue typically of a compact or spongy nature $(50 \mathrm{~cm})$, osteons and haversian canals $(100 \mathrm{~mm})$, fibre patterning and alignment $(50 \mathrm{~mm})$, fibril arrays constituting the fibre $(10 \mathrm{~mm})$, mineralisation of collagen fibrils $(1 \mathrm{~mm})$, formed tropocollagen proteins (300 $\mathrm{nm}$ ) which are made up of amino acids (1 nm) [5]. Each level of hierarchy has been associated with various physical properties; for instance, fibril crack bridging at the micro-scale is one mechanism for increasing toughness, and stiff mineral platelets at the nano-scale deform in tension to transfer stress between discontinuous mineral platelets providing gracious failure. By using principles of such biological materials with current accessible synthetic systems, this may enable the creation of the next generation of man-made composites with improved properties and functionality [6].

Unlike natural composites, current man-made composites have a reinforcing fibre that is typically continuous throughout a component in order to have uninterrupted load transfer [7]. However, this can often result in limited manufacturability $[8,9]$ and a loss in the number of levels of hierarchy. As such, strength and ductility in current composite materials, such as glass or carbon fibre epoxy, are usually mutually exclusive [10]. Current composites therefore tend to lack gracious failure and perform in a brittle manner leading to sudden in-service failure. In engineering, this often means overdesign of components and an increased safety 
factor, increasing mass and cost of the components. Discontinuous man-made composites on the other hand have been shown to increase ductility, particularly for brittle matrices such as epoxy or polyester [9]. However, in-plane properties matching that of continuous fibre composites have yet to be achieved.
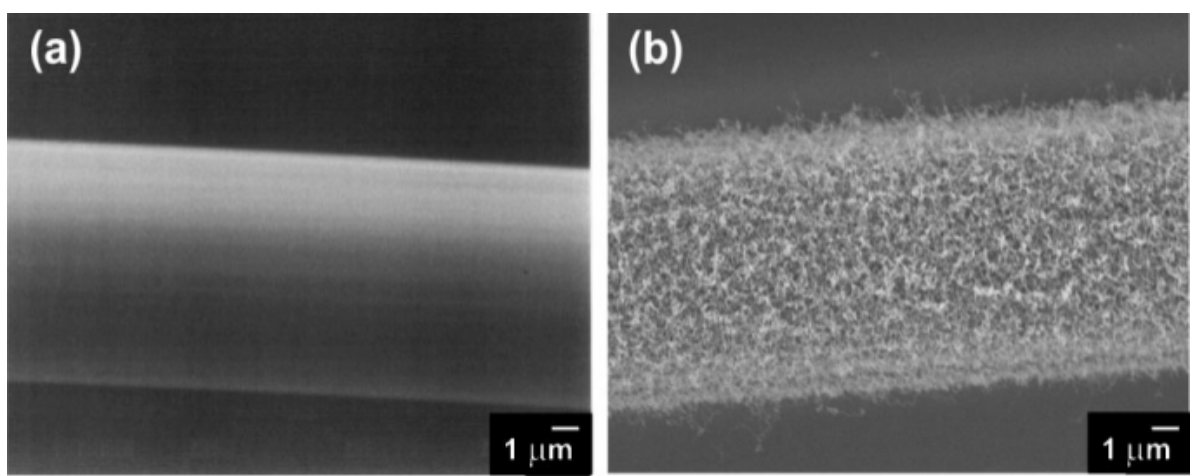

Figure 4.2: Carbon nanotubes grafted onto a carbon fibre surface through a CVD process. a) Bare fibre, b) CNT coated fibre [12].

Considerable efforts have been made to increase the level of hierarchy in composites through the use of nanomaterials alongside typical micro-scale reinforcements, with particular interests in carbon nanotubes (CNT's) due to their intrinsic high strength and stiffness [11]. When CNT's have been incorporated into a matrix several properties have been shown to improve. However, clear improvement of in-plane mechanical properties has yet to be demonstrated [3]. Current mechanical limitations of CNT's include the relatively poor quality of supply, difficulties in their dispersion and alignment within the matrix, and poor bonding between the CNT's and the matrix. CNT's however have been shown to provide other functional advantages such as electrical conductivity, thermal conductivity, increasing service temperature and flame retardancy [3]. To solve some of the mechanical limitations, interest has grown in incorporating CNT's via grafting to the fibre surface through a chemical vapour deposition (CVD) process, before embedding them in a polymer [12] as shown in Figure 4.1. By grafting the nanoscale reinforcement onto the surface of a fibre, alleviation of agglomeration and alignment issues becomes possible, whilst improvements in through thickness properties of a composite have also been demonstrated [13]. It is well known that 
the performance of a composite not only relies on the properties of the individual constituents, but also the interfacial properties between the fibre and the matrix [14]. Alteration of the surface of the fibre can increase load transfer between the matrix and the fibre, either through bonding or mechanical interlocking; however, this is not always desirable. Increased load transfer may induce larger amounts of fibre fracture in a brittle fibre / brittle matrix combination, and hence yield brittleness in the material, a property that well utilised carbon fibre / epoxy resins already exhibit. Hitherto, a relatively ductile fibre coating with enhanced mechanical interlocking remains unknown.

Zinc Oxide $(\mathrm{ZnO})$ nanorods are a promising candidate due to their intrinsic ductility compared with CNT's, with a measured stiffness of approximately $29 \pm 8 \mathrm{GPa}$ [15], which is still greater than that of typical epoxy of 3-4 GPa. The exact value of the modulus however is still disputed and has been shown to vary depending upon the nanorod dimensions $[16,17]$. The relative ease and variety of grafting / growth methods is also a benefit for production purposes. It is common to have a two stage process, first seeding the surface, followed by growth of rods [18]. Growth can be performed hydrothermally in a non-hazardous, low temperature environment. There has been profound interest in $\mathrm{ZnO}$ nanorods of late mainly due to a large range of high technology electronic applications such as field effect transistors [19], flexible electronics [20], light emitting diodes [21], energy scavenging [22], humidity sensing [23], and solar cells [24]. These would offer fertile ground for exploiting added functionalities to composites by incorporating $\mathrm{ZnO}$ nanorods. Several morphologies of nanorods have been documented [25-28], showing a plethora of rod density and alignment. We thus postulate that $\mathrm{ZnO}$ nanorod-coated fibres, when incorporated in a composite, facilitate mechanisms for not only graceful mechanical failure, but also improved load transfer between discontinuous fibre composites. In this work, $\mathrm{ZnO}$ nanorods have therefore been hydrothermally grown onto flat glass surfaces in order to optimise the method for controlling the rod morphology. Subsequently, nanorod growth on a continuous (long) and then a discontinuous (short) glass fibre surface has also been performed. We then investigate, using an atomic force microscopy (AFM) and friction measurement capability at Bristol Chemistry, interfacial shear properties between nanorod-coated surfaces. These results provide 
the first concrete data for us to critically assess the prospect of nanorod-coated fibres for improved ductility in composite materials.

\subsubsection{Discontinuous fibre composites}

Discontinuous composites are rarely used for primary structure components, but more so for secondary structures due to their ease of manufacture $[8,9]$. The processing methods often reduce manufacturing time scales whilst also providing potential for novel manufacturing techniques such as additive layer manufacturing (ALM) [29]. Discontinuous composites do however tend to suffer from inferior stiffness and tensile strength, often caused through poor fibre alignment, fibre lengths below the critical fibre length, Ic, and the difficulty in obtaining high fibre volume fractions [30]. The most widely used model to describe stress transfer in a short fibre composite is the shear lag model, focusing on tensile stress transfer between the fibre and matrix through interfacial shear stress (IFSS) [14]. This leads to the critical fibre length, Ic, which is the minimum length of fibre required for the fibre to fracture through IFSS transfer [14].

In general a high fibre content is required in order to achieve a higher performing short fibre composite [31], and increasing volume fraction (Vf) has been shown to linearly increase fracture toughness [9]. Increasing fibre content has however been found to reduce failure strain, with failure initiation often occurring at fibre ends and propagating across the fibre/matrix interface and finally through the matrix. Modelling has revealed that fibre ends act as stress concentrations with a factor of 10 or higher [31], relating failure to the number of fibre ends, with stress often relieved through matrix flow, interfacial debonding, or matrix failure. The interface is dependent not only on the chemical bonding but also the surface topography, and should be controlled in a manner very specific to a particular fibre/matrix combination. It is frequently the determining factor for the type of fracture mechanism observed [9]. By altering the interface with the application of ZnO nanorods, enhanced load transfer between discontinuous fibres may be possible, whilst the alteration of crack propagation and increased fracture toughness also has potential. 


\subsubsection{Grafting of CNT's to a fibre surface}

Grafting CNT's onto the fibre surface has been shown to improve mechanical properties such as IFSS by approximately 15\% [12]; however, fibre tensile strength is often reduced by approximately $15-55 \%[3,32]$. This is primarily due to the chemical vapour deposition (CVD) method used for growth, where a metallic catalyst is used to 'seed' the surface for CNT growth. It is thought that this seeding method damages the surface, effectively etching away the fibre surface resulting in surface pitting which causes a micro-scale stress concentration, leading to lower tensile strength [3]. Wettability of the CNT forests on the surface has also been a concern, with morphology of the CNT forest and the viscosity of the resin having a large impact. It has been shown that CNT's with a length of $30 \mu \mathrm{m}$ grown in square arrays had difficulty in wetting when using an epoxy resin system. This caused large contraction of the square arrays and large central voids, however, when a lower viscosity was used with a similar CNT array no signs of voidage were present [33]. A measurement of contact angle has also been made using a lower viscosity resin and a CNT length of approximately 2-10 $\mu \mathrm{m}$. This showed a reduction in contact angle with poly(methyl methacrylate) (PMMA) polymer, indicating improved wettability through improved surface energy after CNT growth [13]. Longer growth times in the CVD quartz reactor improved coverage and also improved wetting to the point where the PMMA became fully dispersed on the surface. Investigation also highlighted the kinetics of infiltration; the resin flowed through narrow channels between the CNT's, with increasing resin viscosity indicating a slower diffusion rate. Here, strong bonding between the CNT and the fibre was also shown through a fibre fragmentation test, reducing fibre fragmentation lengths indicative of improved IFSS [13].

CNT growth on the surface of preformed fibres in a composite has also shown a through thickness property improvement; something which is desirable for damage tolerance and reductions in interlaminar failure. CNT growth on a 3D silicon carbide weave showed interlaminar fracture toughness improvements of $348 \%$ in mode I and 54\% in mode II [34], as shown via illustration in Figure 2. In a different experiment on a unidirectional carbon fibre composite, flexural strength as well as modulus were improved by $20 \%$ and $28 \%$ respectively for a CNT coated preform [35]. Dependent on the length of the nano growth, they could be considered as both 
intra-laminar and inter-laminar reinforcement providing resistance to delamination $[34,36]$. Through a three point bend test, some have found that interlaminar shear strength is increased by $69 \%$ after growth [37].

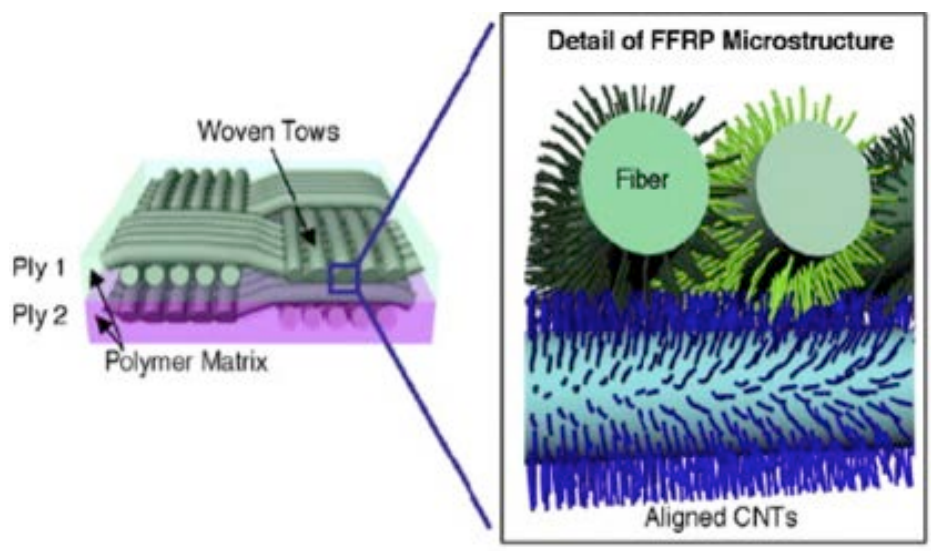

Figure 4.2: Illustration of 'fuzzy' fibres through radially aligned CNT growth on a woven fibre cloth

CNT's have also been grafted to the surface of discontinuous glass fibres and then embedded within a polypropylene (PP) resin. It was found that the in-plane tensile strength of the discontinuous composite was improved by $40 \%$ compared with a bare glass fibre PP composite, whilst the impact strength of the composite was also found to be improved by $24 \%$ [38]. Post failure analysis indicates the fibres pull out with increased adhesion as resin still remains on the surface (Figure 4.3). This is of particular interest due to their potential application in ALM through the addition of short fibres to the feedstock.
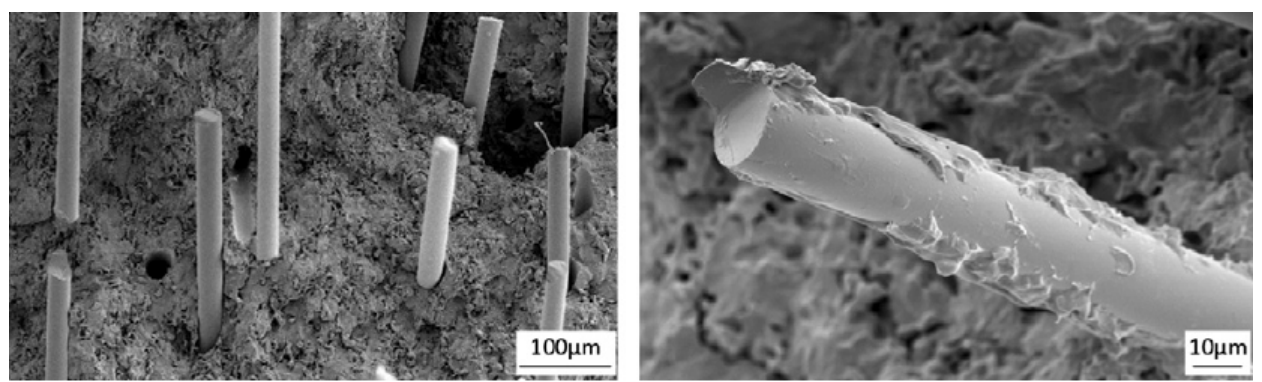

Figure 4.3: SEM images of a) Smooth surface of neat glass, b) PP sticking to the fibre surface [38] 


\subsubsection{Zinc oxide nanorod growth}

$\mathrm{ZnO}$ nanorods have been chosen in this work due to their intrinsic ductility and higher modulus (29 GPa) compared with typically used matrix materials (3-4 GPa). Compared with CNT's (1,000 GPa), the lower modulus of $\mathrm{ZnO}$ can still provide load transfer between discontinuous fibres while allowing ductile failure. ZnO nanorods have typically been grown on silica substrates previously [18], however, glass has a similar surface chemistry with hydroxyl groups required for seeding the substrate. ZnO nanorods have only recently been grown onto curved surfaces, primarily optical fibres or fibres for energy harvesting, shown in Figure $4.4[22,23]$. Current uses for $\mathrm{ZnO}$ nanorods take advantage of the large surface area of nanorod arrays as compared with a flat substrate, and thus efforts have been focused on tuning growth methods for the densest growth morphologies. If the dispersion and alignment of the rods can also be controlled, IFSS could be altered and controlled dependent on morphology.

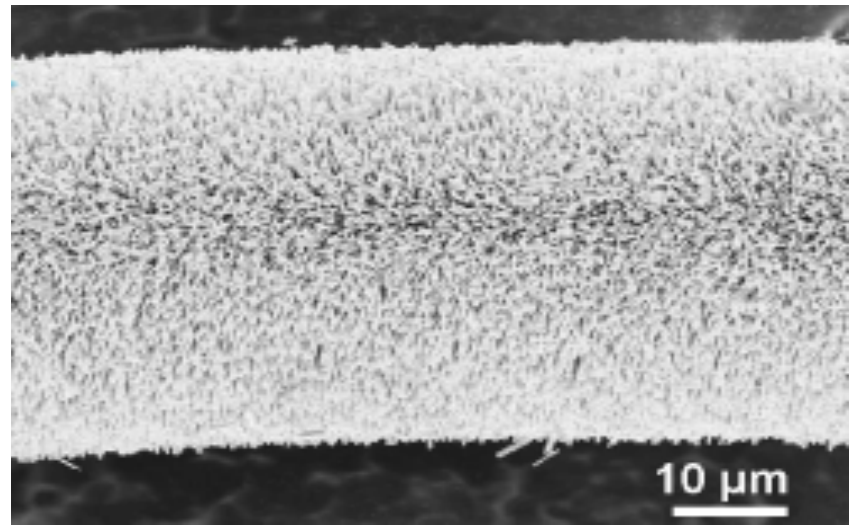

Figure 4.4: SEM image of ZnO nanorod coated kevlar fibre for energy scavenging applications [22].

The typical two-stage process of seeding followed by growth allows for several variable factors with each having an effect on morphology. It has been shown that the seeded method has a large effect on morphology [39, 40], with sputtering or CVD seeding being selected for more control over nanorod alignment as well as producing nanorods with higher aspect ratios [22, 27, 41-44]. However, due to the apparent etching effect caused by the CVD or sputtering processes, a wet chemical seeding method using zinc acetate salts has been used here. The limitation of quartz reactor size or sputtering chamber is also overcome through the use of an 
acetate seed. $\mathrm{ZnO}$ is expected to crystallise through the hydrolysis of $\mathrm{Zn}$ salts in a slightly basic solution, and hence zinc acetate is used with potassium or sodium hydroxide in order for crystallisation to occur at temperature [18]. Ethanol is typically used as the solvent for the acetate basic solution which is used to coat substrates prior to growth. Post coating of the substrate, a thermal decomposition is then required in order to covalently bond the $\mathrm{ZnO}$ to the surface, providing a glass surface chemistry change shown in Figure 5 . Studies have shown that a minimum temperature for thermal decomposition is $150^{\circ} \mathrm{C}$, with higher temperatures around $350^{\circ} \mathrm{C}$ improving perpendicular alignment through a reorientation of planes [45]. Seed particles in ethanol solution vary in size dependent on the molarity of solution, temperature, and incubation time for particles to form. Typical particle sizes expected for a $5 \mathrm{mM}$ zinc acetate solution are 5-15 $\mathrm{nm}$ and TEM results have shown that the c-axis of the crystal wurtzite structure after decomposition on to a substrate surface (Figure 6) are oriented perpendicular to the surface for aligned growth [46]. In order to make sure a substrate is fully coated with zinc acetate seeds, substrates are typically dipped in the solution several times followed by drying, or drop cast and spin coated onto flat substrates several times [20, 23, 28, 39, 47]; with the latter not a feasible option for cylindrical fibres. This is then followed by a high temperature bake to encourage particle-substrate bonding.

Once seeded, a growth method can be selected which will again have an influence on morphology. More control is seen when using CVD as a growth method, allowing for much higher aspect ratios, with rods typically much longer (Figure 7a), up to $120 \mu \mathrm{m}$ [48]. Hydrothermal growth on the other hand provides the prospect of low temperature wet chemical synthesis at low cost. This is the most common method in literature (Figure 7b) [18], and unlike alkali mediated growth for the seed solution, $\mathrm{H} 2 \mathrm{O}$ is the source of oxygen ions required for $\mathrm{ZnO}$ synthesis. Low temperature growth and seeding allows for several substrates to be utilised for growth such as silicon wafers, glass, thermoplastics and organic fibres [18]. An equimolar solution of zinc nitrate hexamethylenetetramine (HMTA) is used in order for growth, with varying molarity across literature from $0.5 \mathrm{M}$ [47] to $4 \mathrm{mM}$ [23]. In the reaction, zinc nitrate supplies Zn2+ ions whilst HMTA, a non-ionic cyclic tertiary amine, readily hydrolyses in water to form $\mathrm{OH}$ - ions to form a basic solution, finally 
combining to produce $\mathrm{ZnO}$ and $\mathrm{H} 2 \mathrm{O}$ after several intermediate reactions (Figure 8). The exact function of HMTA is still relatively unknown [18], however, the hydrolysis is thought to occur gradually, causing a slow release of $\mathrm{OH}$ - ions from water, with a large release causing $\mathrm{ZnO}$ to precipitate out too quickly causing non-oriented growth. Before its decomposition to produce ions, HMTA attaches to the non-polar surfaces of already formed nanorods, promoting longitudinal growth. Several further structures have been observed through seeding after initial growth, followed by growth for a second time; providing secondary smaller rod growth perpendicular to the initial rod growth [49]. Patterned growth can also be observed through targeted seeding of the surface or suppression of seed nucleation sites [41]. This report focusses on ZnO nanorod growth on glass fibres via acetate seeding and hydrothermal growth; with the aim of improving in-plane properties of discontinuous composites whilst allowing for ductile failure.

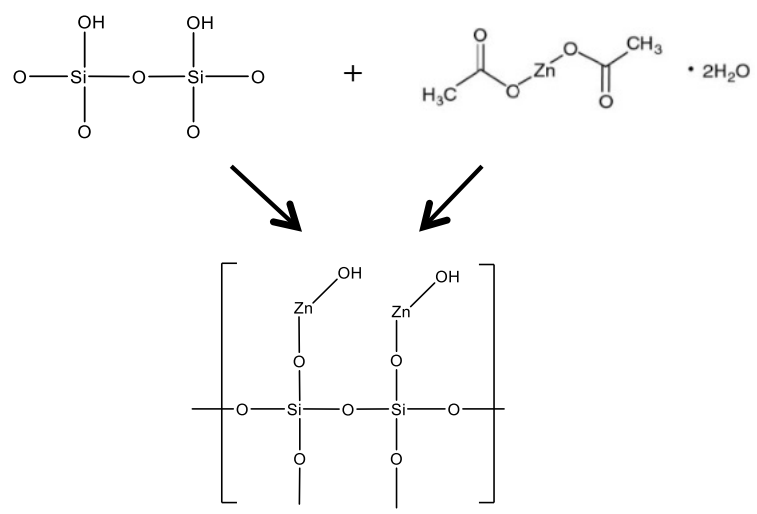

Figure 4.5: Schematic of zinc acetate seeding altering the surface chemistry of glass.

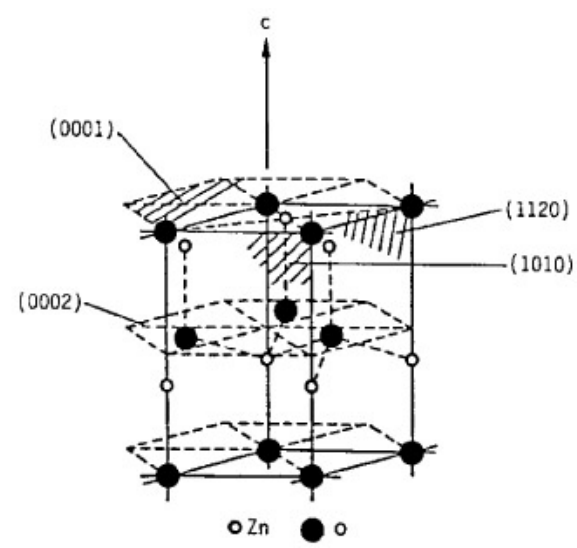

Figure 4.6: Wurtzite crystal structure of ZnO nanorods. 


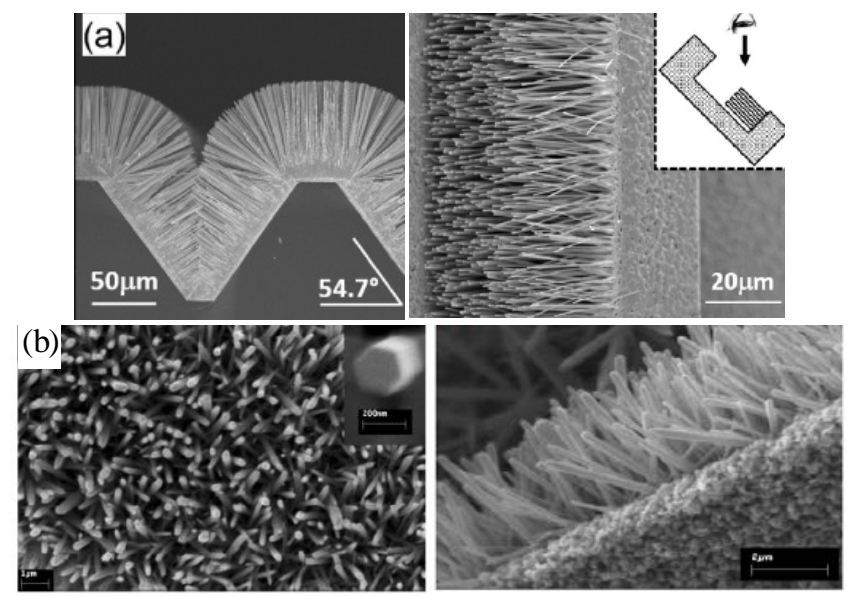

Figure 4.7: SEM images of ZnO nanorods a) CVD seed and growth [48] b) acetate seed and hydrothermal growth [28].

$$
\begin{aligned}
& \mathrm{HMTA}+6 \mathrm{H}_{2} \mathrm{O} \longleftrightarrow 4 \mathrm{NH}_{3}+6 \mathrm{HCHO} \\
& \mathrm{NH}_{3}+\mathrm{H}_{2} \mathrm{O} \longleftrightarrow \mathrm{NH}_{4}^{+}+\mathrm{OH}^{-} \\
& \mathrm{Zn}^{2+}+4 \mathrm{NH}_{3} \longleftrightarrow\left[\mathrm{Zn}\left(\mathrm{NH}_{3}\right)_{4}\right]^{2+} \\
& \mathrm{Zn}^{2+}+2 \mathrm{OH}^{-} \longleftrightarrow \mathrm{Zn}(\mathrm{OH})_{2} \\
& \mathrm{Zn}(\mathrm{OH})_{2} \longleftrightarrow \mathrm{ZnO}+\mathrm{H}_{2} \mathrm{O}
\end{aligned}
$$

Figure 4.8: Intermediate reactions for the production of $\mathrm{ZnO}$ nanorods during hydrothermal growth [18].

\subsection{Experimental}

\subsubsection{Materials used}

Glass microscope slides $0.8-1 \mathrm{~mm}$ thick were used as the substrate for morphology control on a flat surface. Discontinuous short chopped glass fibres $3 \mathrm{~mm}$ in length and approximately $12 \mu \mathrm{m}$ in diameter from vetrotextiles, sized with a starch based oil sizing (T30), were used for batch growth. Continuous glass fibres were produced from Duran borosilicate glass with a diameter of approximately 32 $\mu \mathrm{m}$ with no sizing agent for $\mathrm{ZnO}$ growth. Seed solution was produced with zinc acetate dihydrate $(\mathrm{Zn}(\mathrm{CH} 3 \mathrm{COO}) 2.2 \mathrm{H} 2 \mathrm{O}, 99 \%$ purity, Sigma Aldrich), ethanol $(\mathrm{CH} 3 \mathrm{CH} 2 \mathrm{OH}$, absolute, Sigma Aldrich), and ( $\mathrm{KOH}, 99 \%$ purity, Sigma Aldrich). For growth, a mix of zinc nitrate hexahydrate $(\mathrm{Zn}(\mathrm{NO} 3) 2.6 \mathrm{H} 2 \mathrm{O}, 98 \%$ purity, Sigma Aldrich) and HMTA (C6H12N4, $\geq 99.0 \%$ purity, Sigma Aldrich) was used in deionised 
water (MilliQ, 18.2M $\mathrm{M}$ resistivity, < 3ppb TOC). Post growth a tetraethyl orthosilicate (TEOS) (Si(OC2H5)4, 98\% purity, Sigma Aldrich) coating was applied.

\subsubsection{Growth method of ZnO on flat glass surface}

Prior to growth all glass slides were cleaned in $69 \% \mathrm{w} / \mathrm{v}$ nitric acid (Sigma Aldrich). Seeding and growth was performed on the glass microscope slides with an approximate area of $1 \mathrm{~cm} 2$ post nitric acid cleaning. $35.7 \mathrm{mg}$ of $\mathrm{Zn}(\mathrm{CH} 3 \mathrm{COO}) 2.2 \mathrm{H} 2 \mathrm{O}$ was heated for $1 \mathrm{~h}$ at $50^{\circ} \mathrm{C}$ under vigorous stirring in $40 \mathrm{~mL}$ of ethanol $(4 \mathrm{mM}$ solution), whilst separately $12.9 \mathrm{mg} \mathrm{KOH}$ was added to $40 \mathrm{~mL}$ of ethanol (4 mM solution) and heated for $1 \mathrm{~h}$ at $50^{\circ} \mathrm{C}$ under vigorous stirring. Both solutions were left to cool in air for approximately $10 \mathrm{~min}$ followed by mixing through drop wise addition under vigorous stirring. The $4 \mathrm{mM}$ combined solution was heated at $70^{\circ} \mathrm{C}$ for $2 \mathrm{~h}$ under vigorous stirring and then bottled. Seeding was performed through dipping the cleaned glass slides into the solution for $15 \mathrm{~min}$ at a time, and then placing them on a hot plate at $150^{\circ} \mathrm{C}$ for $15 \mathrm{~min}$ after removal from the solution. This process was repeated 1-5 times in order to investigate the dependency of morphology on the density of the seed. After coating the substrates, they were baked at $310^{\circ} \mathrm{C}$ on a hot plate for $2 \mathrm{~h}$. A growth solution was then prepared in 125 $\mathrm{mL}$ of deionised MilliQ water with $185.9 \mathrm{mg}$ of $\mathrm{Zn}(\mathrm{NO}) 2.6 \mathrm{H} 2 \mathrm{O}$ and $87.6 \mathrm{mg}$ of HMTA (5 mM solution). Before growth, the solution was stirred vigorously for $30 \mathrm{~min}$. Substrates were then placed in the growth solution at $93^{\circ} \mathrm{C}$ under reflux for $8-36 \mathrm{~h}$ (SI Figure 1), with growth time varied to investigate its effect on morphology. After growth, substrates were removed from the solution and rinsed with deionised MilliQ water to remove any salts and heated for $15 \mathrm{~min}$ at $150^{\circ} \mathrm{C}$ to remove any remaining water. Finally a TEOS coating was applied through dipping the substrate in a TEOS solution for 2-3 min followed by drying on a hot plate at $150^{\circ} \mathrm{C}$.

\subsubsection{Growth method of $\mathrm{ZnO}$ on short e-glass fibre}

Short e-glass fibres $3 \mathrm{~mm}$ in length initially had a starch based oil sizing which was removed through sonification in ethanol for $1 \mathrm{~h}$, followed by three 15 min UV ozone treatments. This was done twice to ensure polymer removal. This opened up hydroxyl groups on the glass surface required for bonding, whilst also separating the 
fibres after they were bundled together post sizing (SI Figure 2). An additional step required to remove the fibres from solution was filtration under vacuum, which occurred after sonification (SI Figure 3), after each seeding step, and after growth. Creation of the seed and growth solution remained the same as in Section 2.2. Seeding of the fibres remained constant at $3 \times 15$ min dipping and heating cycles, followed by a $310^{\circ} \mathrm{C}$ bake. Growth solution concentration was varied from 5 to 10 $\mathrm{mM}$ to account for a large increase in growth surface area. $0.05 \mathrm{~g}$ of fibres were batch processed at a time in order to control surface area and dispersion. After growth, fibres were removed from the solution and rinsed with deionised MilliQ water to remove any salts and then heated for $15 \mathrm{~min}$ at $150^{\circ} \mathrm{C}$ to remove any remaining water. Finally a TEOS coating was applied through dipping the fibres in a TEOS solution for 2-3 min followed by drying on a hot plate at $150^{\circ} \mathrm{C}$.

\subsubsection{Growth method of $\mathrm{ZnO}$ on continuous fibre}

An individual borosilicate glass fibre $6 \mathrm{~cm}$ in length was seeded and grown on through being placed in a mounting fixture and clamped at either end (SI Figure 4.4). Creation of the seed and growth solution remained the same as in Section 2.2. Once in the mounting fixture it remained there until each step of seeding and growth had been completed. This meant that growth could not be performed in a round bottom flask under reflux, and hence had to be performed in a beaker (SI Figure 4.5). A growth solution concentration of $5 \mathrm{mM}$ was used for $8 \mathrm{~h}$ of growth. Deionised water was added to the solution on the hour in order to maintain growth solution concentration. After growth, the fibril was rinsed with deionised MilliQ water to remove any salts and heated for $15 \mathrm{~min}$ at $150^{\circ} \mathrm{C}$ to remove any remaining water. Finally a TEOS is applied by dipping the fibre in a TEOS solution for 2-3 min followed by drying on a hot plate at $150^{\circ} \mathrm{C}$.

\subsubsection{Individual fibre tensile tests}

Single fibre tensile tests were performed at room temperature in accordance to ASTM C1557 [50], with a gauge length of $10 \mathrm{~mm}$ and $30 \mathrm{~mm}$ (SI Figures 4.6 and 4.7). Borosilicate continuous glass fibres were tested before and after $\mathrm{ZnO}$ nanorod growth at a crosshead speed of $35 \mathrm{~mm} / \mathrm{min}$. A $10 \mathrm{~N}$ load cell was used on a $1 \mathrm{kN}$ 
Instron tensile testing machine, whilst a Letoxit epoxy adhesive (SI Figure 4.6) was used to attach the fibril to a supporting card. Four tests were performed at a gauge length of $30 \mathrm{~mm}$ for bare glass fibre, eight tests at $10 \mathrm{~mm}$ of bare glass fibre (SI Figure 4.8), and finally four tests of $\mathrm{ZnO}$ nanorod coated fibres following treatment with TEOS.

\subsubsection{Atomic force microscopy measurements}

AFM measurements of lateral and normal force were taken on a $1 \times$ seeded and $3 \times$ seeded $\mathrm{ZnO}$ nanorod coated surface, before and after a coating of TEOS. It is thought that this would be indicative of the strength of attachment of the nanorods to the substrate surface, whilst also providing some insight into interfacial shear of different nanorod morphologies. Tipless Mikromasch non-coated CSC37 series AFM probes were used with an attached glass fibre, both with and without $\mathrm{ZnO}$ nanorod growth. Lever B (SI Figure 4.9) with a force constant of 0.1-0.6 N/m was selected. Tips were functionalised through particle attachment via micromanipulators using epoxy glue followed by curing. Particles attached to tipless cantilevers are typically spherical [51], and hence this method will provide novel insight into shape effects of the cantilever tip. Normal and torsional spring constants of the cantilevers were determined by thermal vibration methods $[52,53]$. However, this method showed significantly lower flexural stiffness than expected (SI Table 2 ), therefore $0.3 \mathrm{~N} / \mathrm{m}$ was assumed as stated as the average by the Mikromasch manufacturer (SI Figure 4.9). Tips were imaged using a JEOL JSM 6330F for high resolution images, shown in Figure 4.10. Normal force measurements were performed at 3-5 locations across each surface. Lateral force measurements (friction) were recorded over a scan range of $5 \mu \mathrm{m}$ at $1 \mathrm{~Hz}$, providing a shear velocity of $10 \mu \mathrm{ms}-1$, with variable normal force. Height required for calculating torque was assumed as particle diameter plus half the thickness of the cantilever $(1 \mu \mathrm{m})$. Higher velocities have been shown to cause a reduction in friction coefficient [54], likely due to skipping of the tip over the rough surface. Prior to use, substrates as well as tips were cleaned via UV ozone treatment for $15 \mathrm{~min}$. 

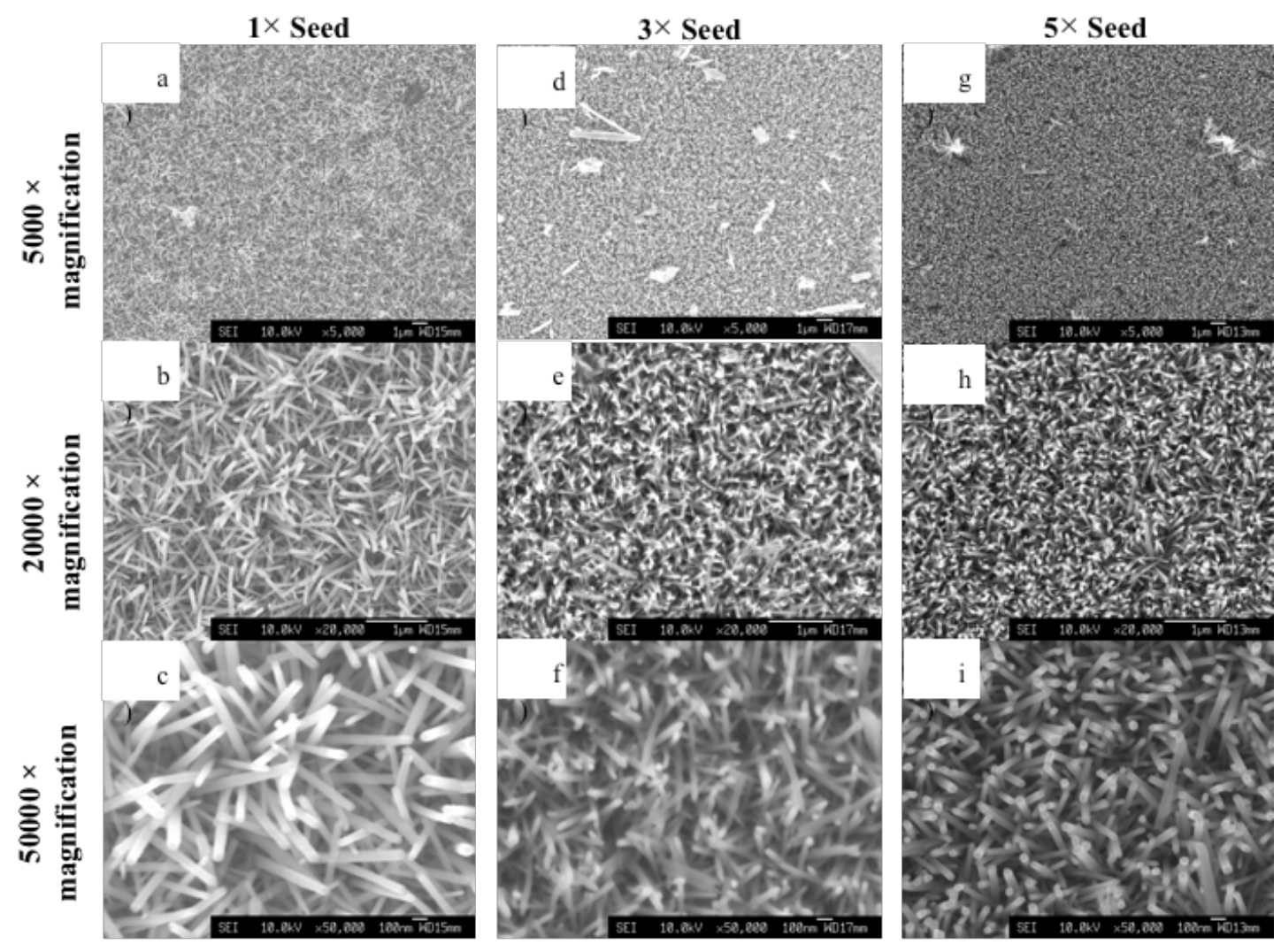

Figure 4.9: SEM images of morphology control of ZnO nanorods through altering the amount of seeding: a-c show 1 repetition of the seeding process, $d-f$ show 3 repetitions of the seeding process, g-i show 5 repetitions of the seeding process outlined in section 2.2. Magnifications remain constant across the horizontal axis. 

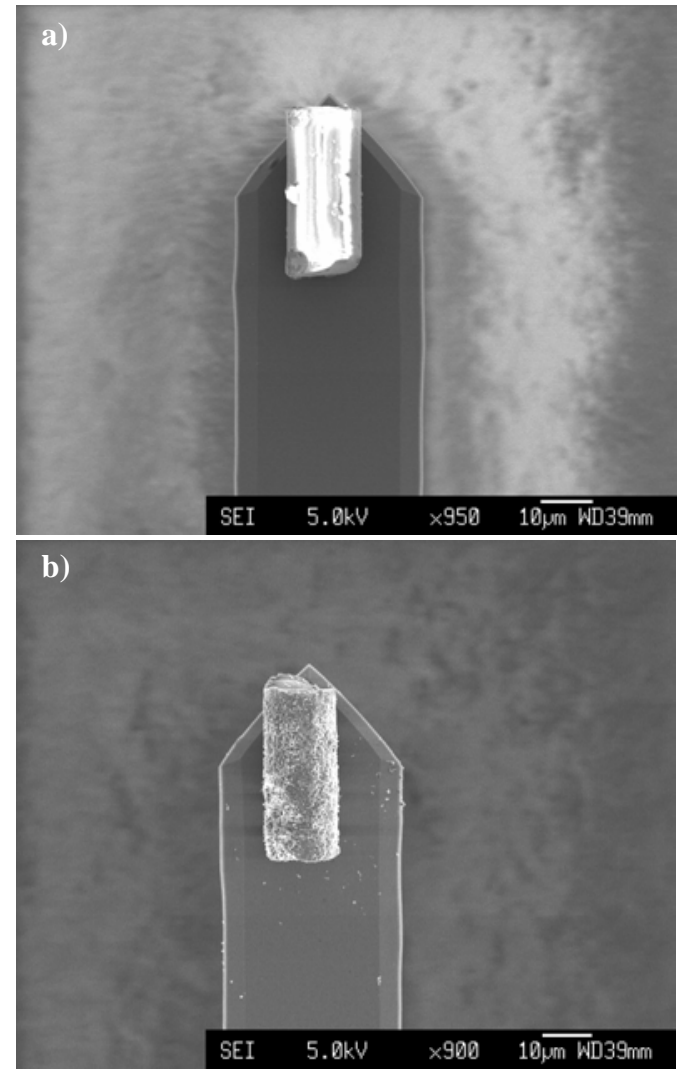

Figure 4.10: SEM images of: a) Glass fibre functionalised tip b) Glass fibre with ZnO nanorod growth functionalised tip.

\subsection{Results and discussion}

\subsubsection{Controlling the morphology of $\mathrm{ZnO}$ growth on a flat surface}

The number of repetitive seeding cycles on a $1 \mathrm{~cm}^{2}$ substrate was varied 1-5 times followed by a $310^{\circ} \mathrm{C}$ bake, whilst growth conditions initially remained constant with a $5 \mathrm{mM} \mathrm{HMTA}$ and zinc nitrate solution at $93^{\circ} \mathrm{C}$ for $8 \mathrm{~h}$. As seen in Figure 4.9, increasing the seed density increased nanorod density whilst also improving alignment. It is also seen that at lower seed densities nanorods were slightly larger at approximately $100 \mathrm{~nm}$ in diameter compared with $50 \mathrm{~nm}$ for the 3 and 5 times seeded substrates. This is thought to be due to the reduced nucleation sites for growth causing increased deposition on the remaining growth sites. Once the seed layer reaches a certain thickness, rod growth impedes adjacent rods from forming at an angle to the surface. The highest density is likely to be undesirable for wetting, so a $3 x$ seeding approach was selected for fibre growth. The length of nanorods was also imaged through cracking the substrate in half, as shown in Figure 4.11, to be 
approximately $1 \mu \mathrm{m}$ in length. It was found that varying growth time at $8 \mathrm{~h}, 22 \mathrm{~h}$ and $38 \mathrm{~h}$ had no effect on morphology and did not increase nanorod length; indicating that the nutrients in solution were depleted after $8 \mathrm{~h}$ of growth. It is thought that length can be extended through increasing concentration, or the addition of further reactants for growth after a certain time period.

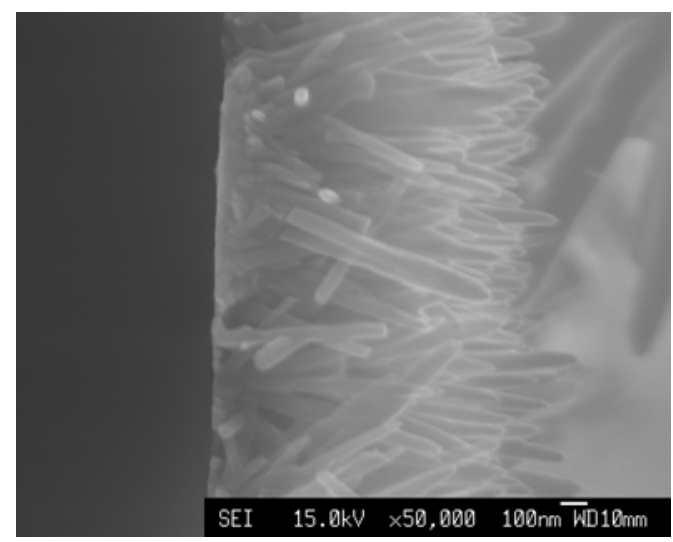

Figure 4.11: SEM image of $3 x$ seeded substrate grown for $38 \mathrm{~h}$ in $5 \mathrm{mM}$ growth concentration.

It was also noted that morphology varied from site to site on a particular substrate, as shown in Figure 4.12. It can be seen that regions of higher alignment are well pronounced as are regions with lesser alignment; however, with increased seeding the regions of poorer alignment tend to diminish. Variation of substrate surface area was also investigated, indicating a morphology dependence that is so far not reported in literature. Larger rods were found to be present at $10 \mu \mathrm{m}$ in length after halving the substrate surface area; however they were not perpendicular to the surface and not clearly bonded to the surface (SI Figure 4.10).

X-Ray Diffraction (XRD) is usually performed at this point to confirm the degree of orientation as well as foreign particle content [28]; however, due to time constraints this was not performed. A high resolution image clearly shows the hexagonal structure of the $\mathrm{ZnO}$ nanorod (Figure 4.13); typical of the wurtzite crystal structure. 


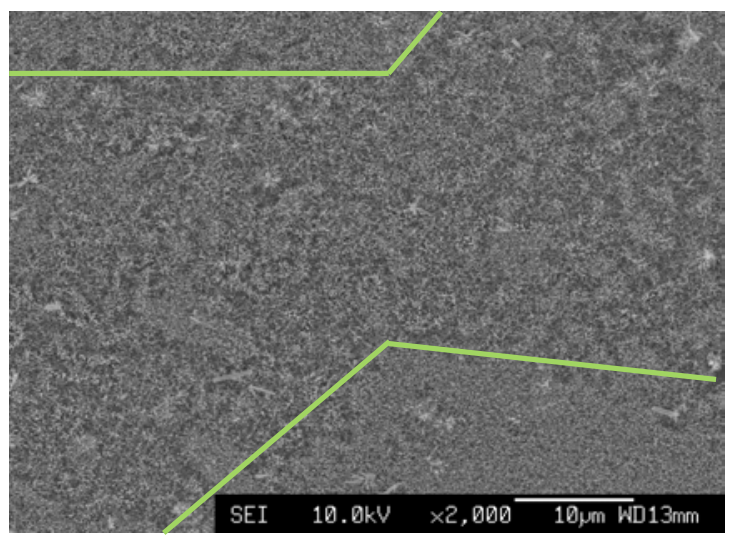

Figure 12: SEM image of 4 times seeded sample, with lines indicating regions of different alignment.

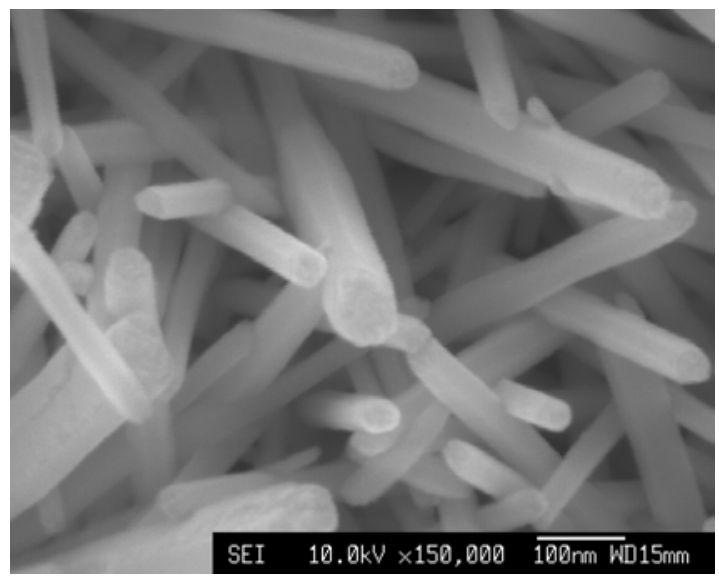

Figure 13: SEM image of ZnO hexagonal crystal wurtzite structure.

\subsubsection{ZnO nanorods on glass fibres}

After initial flat substrate testing, a cycle of 3 seeding steps was selected for growth on short glass fibres $3 \mathrm{~mm}$ in length. As with flat glass substrate cleaning, concentrated nitric acid cleaning was initially employed for removal of the starch based polymer sizing; however, it was found to damage the surface of the glass and caused loss of nanorods after growth (Figure 4.14). During this stage it was also noted that the initial $5 \mathrm{mM}$ concentration of HMTA and zinc nitrate hexahydrate for growth was insufficient for the surface area requiring growth (SI Table 1); $0.05 \mathrm{~g}$ of fibres has an approximately 20 times increase in surface area compared with previous flat substrate growth. The growth solution was therefore increased in concentration to $10 \mathrm{mM}$, whilst sonification in ethanol followed by UV treatment was selected for removal of the polymer.

\section{Distribution A: Approved for public release; distribution is unlimited.}


Good coverage was noted on glass fibres after an increase in growth concentration as well as swapping cleaning methods (Figure 4.15). However, fibres began to agglomerate in the growth solution and bundles of glass fibres formed; even whilst under vigorous stirring (SI Figure 4.11). Central areas of the bundles show poor nanorod growth, while outer areas exposed to nutrients received excellent coverage and growth. Approximate $\mathrm{ZnO}$ nanorod aspect ratio remained similar to that on flat glass substrates and perpendicular to the surface (SI Figure 4.12). It is also seen from Figure 4.15 that there is an increase in the deposition of the nanorods on the surface that have formed in solution. Flat glass slides were easily cleaned with MilliQ water; however, filtration under vacuum to remove fibres from solution also removes longer nanorods that have grown in solution. This makes cleaning of the fibre bundles more difficult; however, rinsing was still conducted several times whilst fibres remained on the fine glass Buchner funnel. It is very difficult to avoid bundling during growth as open hydroxyl surface groups are required for $\mathrm{ZnO}$ nanorod surface bonding, and also cause hydrogen bonding of the glass fibres.

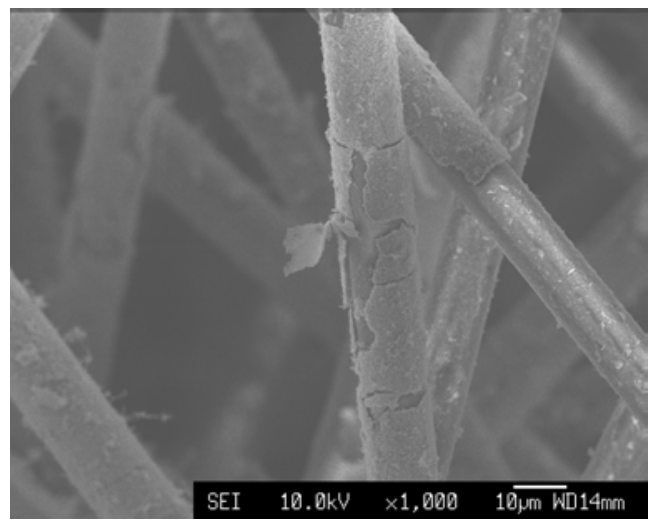

Figure 4.14: SEM image of the loss of ZnO nanorods from the glass fibre surface due to concentrated acid cleaning. 


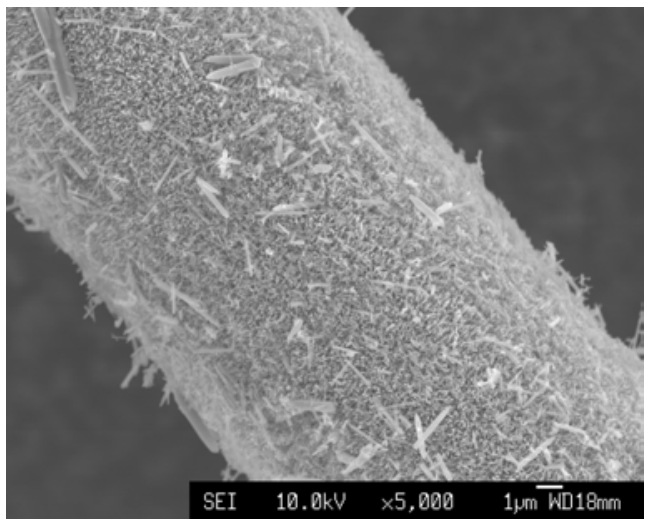

Figure 4.15: SEM image of 3x seeded short glass fibre, $10 \mathrm{mM}$ growth concentration in a batch process.

In order to counter the agglomeration problem as well as any unforeseen consequences of polymer sizing removal, continuous borosilicate glass fibres $6 \mathrm{~cm}$ in length were produced without any sizing. A larger diameter was produced of approximately $33.28 \mu \mathrm{m}$ in diameter (SI Figure 4.13), where before and after growth can be seen in Figure 4.16. Distribution is noted to be sparser than previously grown rods on both a flat glass surface and short glass fibres (SI Figure 4.17). This is thought to be due to the poor temperature seeding; the rig setup does not allow an oven bake and cannot be placed in direct contact with the hot plate surface. Therefore, although the hot plate temperature was set at a maximum, the fibre temperature was considerably lower. The quality of the seed has been previously shown to be dependent on temperature [45], and hence a custom-designed holding device will be required to further the potential of this method that allows temperatures up to $350^{\circ} \mathrm{C}$ within a furnace. Due to the fewer nucleation sites, nanorods have grown with an approximate aspect ratio of 10; considerably less than the aspect ratio of 20 seen on flat glass substrates and short glass fibres. Contaminants can also be seen on the fibre surface, which is thought to be due to the oxidation of the clamping equipment. 

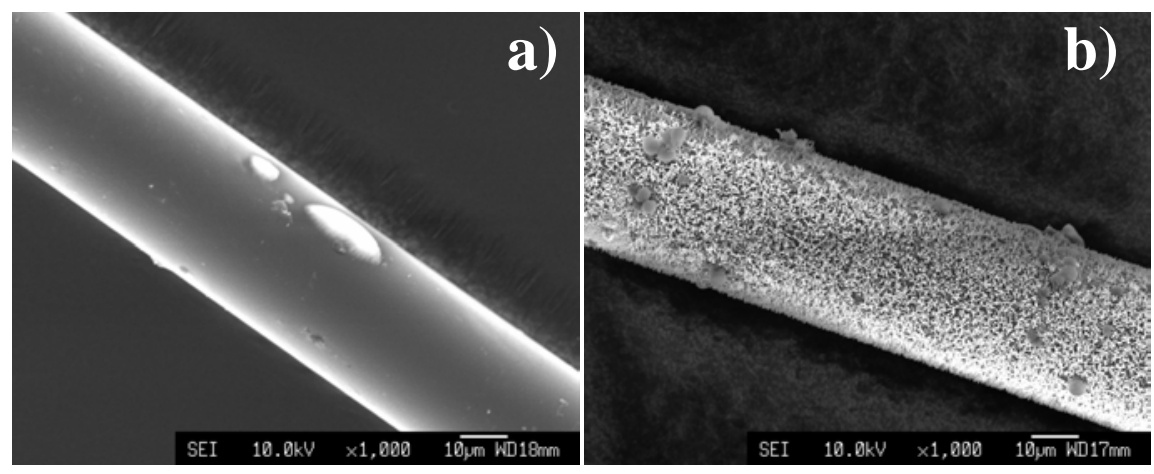

Figure 4.16: a) SEM image of glass fibre prior to ZnO nanorod growth indicating a fibre diameter of 32.81 $\mu \mathrm{m}$, b) after ZnO growth.

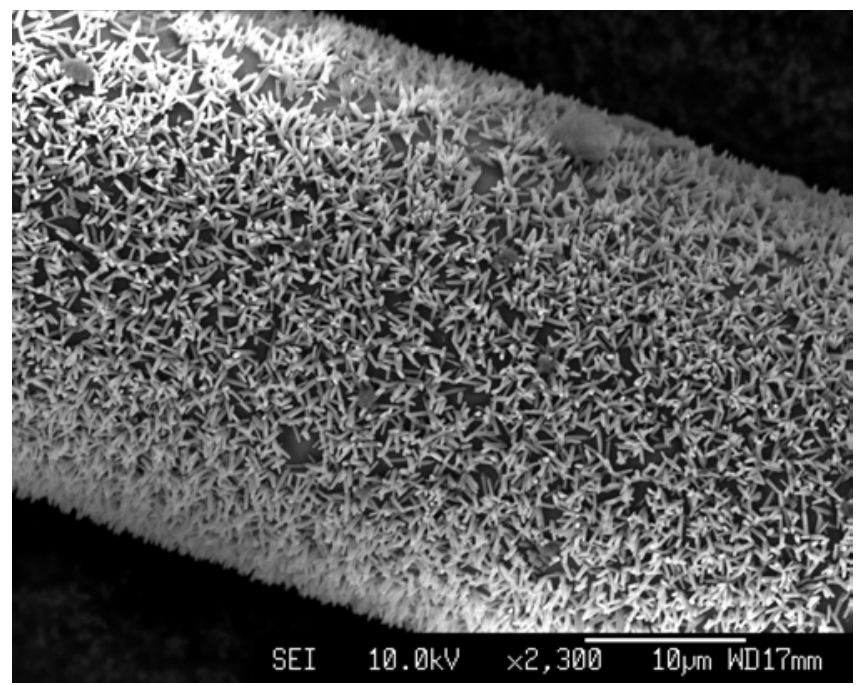

Figure 4.17: SEM image of continuous fibre ZnO nanorod growth, indicating sparser arrangement of nanorods.

In order to ensure the $\mathrm{ZnO}$ nanorods are securely seated on the fibre surface, a TEOS coating is applied. TEOS, often used as a cross linking agent or to make pure silica [55] (Figure 4.18), cross links on the surface when combined with moisture in the atmosphere. This in effect embeds the roots of the $\mathrm{ZnO}$ fibre to the glass surface in silica [22] (Figure 4.19). Through increasing surface roughness and contact area, nanorods or nanotubes are expected to draw the non-viscous TEOS to the roots through capillary action [13, 22, 29]. A brief investigation of SEM before and after TEOS coating revealed no difference in imaging, ensuring that TEOS had not been left on the surface covering nanorods (SI Figure 4.14-4.15). 


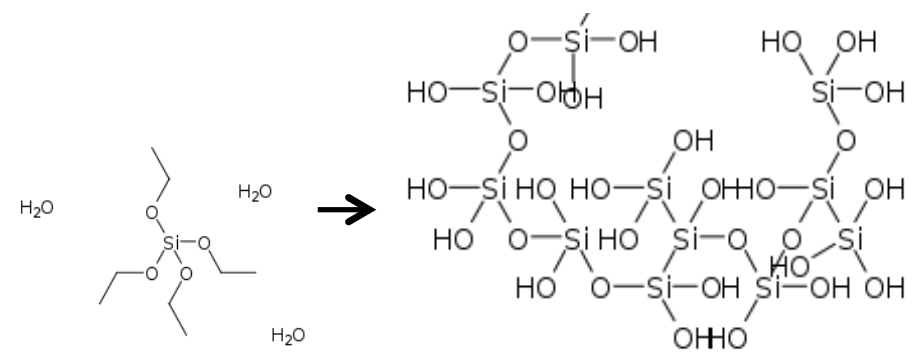

Figure 4.18: TEOS cross linking in the presence of $\mathrm{H}_{2} \mathrm{O}$.

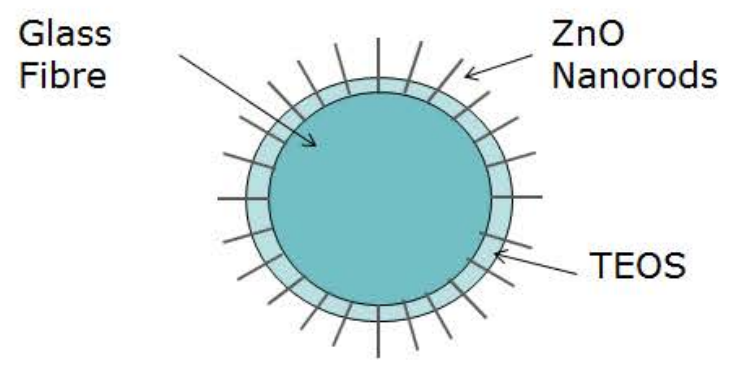

Figure 4.19: Illustration of TEOS coated fibre cross section; not to scale.

\subsubsection{Individual fibre tensile testing}

Individual fibre tensile tests were performed on the produced continuous borosilicate glass fibre, approximately $33.281 \mu \mathrm{m}$ in diameter (SI Figure 13), before and after $\mathrm{ZnO}$ nanorod growth. This was performed to understand if the attachment method had any unexpected detrimental effects on tensile strength similar to that of the CVD approach seen for CNT growth. An initial gauge length of $30 \mathrm{~mm}$ was chosen, followed by $10 \mathrm{~mm}$ in order to reduce the probability of premature failure. The results of tensile strength are given in Figure 20. The lowest tensile strength average of $278 \mathrm{MPa}$ was seen at a gauge length of $30 \mathrm{~mm}$, whilst reducing gauge length to $10 \mathrm{~mm}$ increased the average tensile strength to $336 \mathrm{MPa}$. As seen from the SEM in Figure 16(a), there are several defects in the glass produced, having a pronounced effect on the fibre tensile strength [14]. By reducing gauge length by a third the probability of inclusion of a limiting strength defect in the glass is also reduced by a third. At a gauge length of $10 \mathrm{~mm}$, the average strength found differed by only $4 \%$ from literature where identical glass and production procedures were used [56]. Tensile strength following ZnO growth and a TEOS coating showed an 
average increase to $373 \mathrm{MPa}$; clearly indicating that the growth procedure is not detrimental to the tensile properties of the borosilicate glass but in fact provides an approximate $11 \%$ increase in strength. This is thought to be due to the TEOS coating following growth; where the TEOS coating 'heals' some of the imperfections shown in Figure 16(a), reducing stress concentration factors imparted on the fibre from the defects. Also expected is a slight cross sectional area increase (Figure 19); however a $3.3 \%$ increase in radius relating to an $11 \%$ increase in area seems unlikely. In order to confirm this, a further comparative study would be required on the tensile strength of bare glass fibres with just a TEOS coating. Young's modulus was also calculated to be $65 \mathrm{GPa}$ after $\mathrm{ZnO}$ growth, slightly higher than anticipated with Duran Borosilicate glass expected to have a modulus of 63GPa. Raw data is given in SI Figures 14-16.

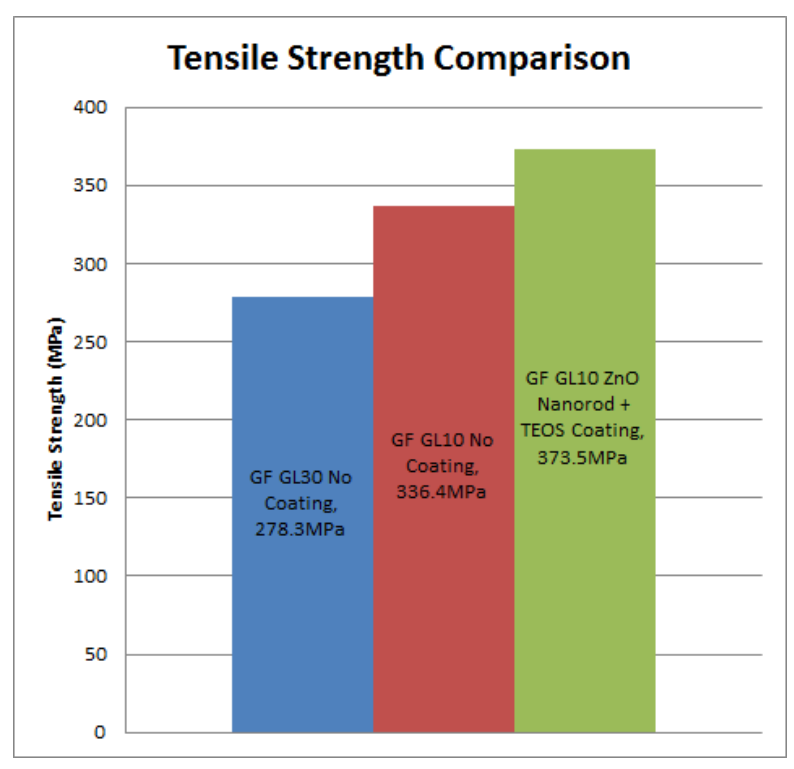

Figure 4.20: Comparative single fibre tensile strengths at $30 \mathrm{~mm}$ gauge length, $10 \mathrm{~mm}$ gauge length and $10 \mathrm{~mm}$ gauge length with ZnO nanorod growth and a TEOS coating.

\subsubsection{AFM measurements}

Normal force data was taken to ensure the cantilever was operating within its linear range of the photodiode and inspect any adhesion forces present. This data also provided the cantilever sensitivity $(\mathrm{nm} / \mathrm{V})$ and the zero load offset for deflection; calculated each time the AFM tip probe was re-engaged to the substrate surface. Glass against a bare glass fibre was analysed first for a base comparison, indicating good separation of trace and retrace curves (Figure 4.21). Roughness of 
the surface is shown through peaks and troughs; with the trace and retrace appearing similar (i.e. of mirror image of each other), suggesting little skipping of the probe due to high velocities. A coefficient of friction was calculated according to Amontons' laws of friction [57], taking an average lateral force from the trace and retrace from 16 loading conditions from 0 to $95 \mathrm{nN}$. Friction can then be plotted against normal load and a linear best fit applied to provide the friction coefficient (Figure 4.22). Figure 4.22 shows a difference between loading and unloading averages which tend to increase at lower normal force loading. This may be due to a smaller normal force leading to a slower reaction and/or poor surface contact following asperities on the surface. A friction coefficient of 3.24 was calculated, higher than previously published results of AFM tip's against pure silicon of 1.07 [54]. It is thought that this is due to errors in the spring constant stiffness and torsional calculation (which will be checked) however, results compared here will be relative and thus internally consistent.

The $3 \times$ seeded substrates were then investigated prior to and after a TEOS coating in order to compare adhesion. Particle contact area estimation is difficult due to the angle of the tip, its shape, and approximate length; and hence only an estimate of contact pressure is given here. Assuming a contact area of a 6th of the projected area of the cylinder, a contact pressure of approximately $3.4 \mathrm{kPa}$ is applied at a maximum load of $95 \mathrm{nN}$. This is very small compared with stress expected to be transferred through IFSS in a composite; however true contact area is expected to be lower due $\mathrm{ZnO}$ nanorod asperities. This causes regions of higher local stress, whilst the differences in the trace and retrace have potential to indicate a possible direction dependence of friction due to anisotropic alignment of rods. It is also possible that a difference in trace and retrace is due to $\mathrm{ZnO}$ nanorod cleavage from the surface. Raw traces at $95 \mathrm{nN}$ of applied load of $3 \times$ seed without and with TEOS can be seen in Figure 4.23 and Figure 4.24 respectively. Both traces indicate a much rougher surface with a large increase in the amplitude of peaks and toughs as expected after $\mathrm{ZnO}$ nanorod growth [54]. Large amplitude peaks of up to $8 \mathrm{~V}$ were often seen in both the trace and retrace largely due to rods that were not aligned perpendicular to the surface. An increase in the asymmetry of peaks of amplitudes at approximately $3 \mathrm{~V}$ was often seen in $3 \times$ seeded growth without TEOS, perhaps 
indicating damage. Furthermore, friction plots of the substrate without TEOS tend to show a drop in friction on unloading providing hysteresis, again indicative of damage (Figure 4.25). Following a TEOS coating, hysteresis is generally eliminated (Figure 4.26). This is shown at several locations across the surface (SI Figure 4.19). The coefficient of friction on average was found to be 6.84 following a TEOS coating; directly comparable to the uncoated $\mathrm{ZnO}$ nanorod growth friction coefficient of 6.97. Following these results all substrates were coated with TEOS for the remainder of testing.

The inter-digitation of nanorod coated surfaces was also of interest. A nanorod coated fibre with $3 \times$ seeded growth was used as a colloidal particle to perform a similar test on the $3 \times$ seeded substrate, both coated in TEOS. Shear traces show good separation of surfaces indicating friction was substantial (Figure 4.27). Peaks and troughs are of a similar frequency and amplitude as in previous shear traces. The friction plots show little to no hysteresis indicating rods are perpendicular to the substrate surface and no damage is observed on the retrace. Friction coefficient is calculated as 5.49, slightly lower than previous measurements of $\mathrm{ZnO}$ nanorod growth against a bare glass fibre. This is thought to be due to the inter-digitation of nanorods causing a reduction in nanorod bending, in turn reducing friction. A smoother surface on the other hand has contact with more rods causing increased rod bending. A reduction in the coefficient of friction between nanorod coated surfaces has been seen previously supporting this result [54].

The $1 \times$ seeded $\mathrm{ZnO}$ growth substrates with lesser alignment were also used in AFM with the hope of identifying a friction dependence on nanorod morphology. However, results showed much lower friction coefficients, thought to be due to contact with larger rods formed in solution connected to the surface (SI Figure 4.20). It is thought that this causes $1 \mu \mathrm{m}$ length nanorods to be out of contact, therefore not contributing to the frictional resistance seen. Thorough analysis has however yet to be performed on this data. 


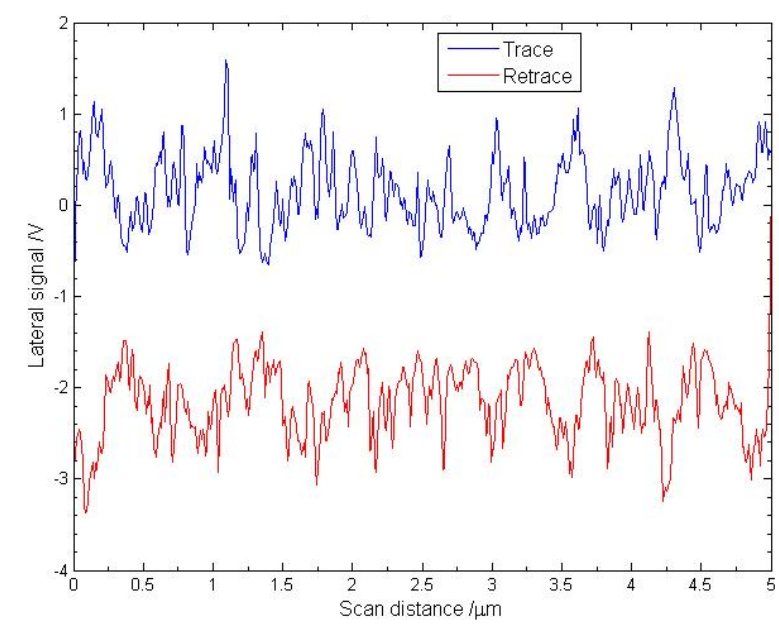

Figure 4.21: Bare glass substrate vs bare glass probe shear trace following $95 \mathrm{nN}$ of force assuming a spring constant of $0.3 \mathrm{~N} / \mathrm{m}$

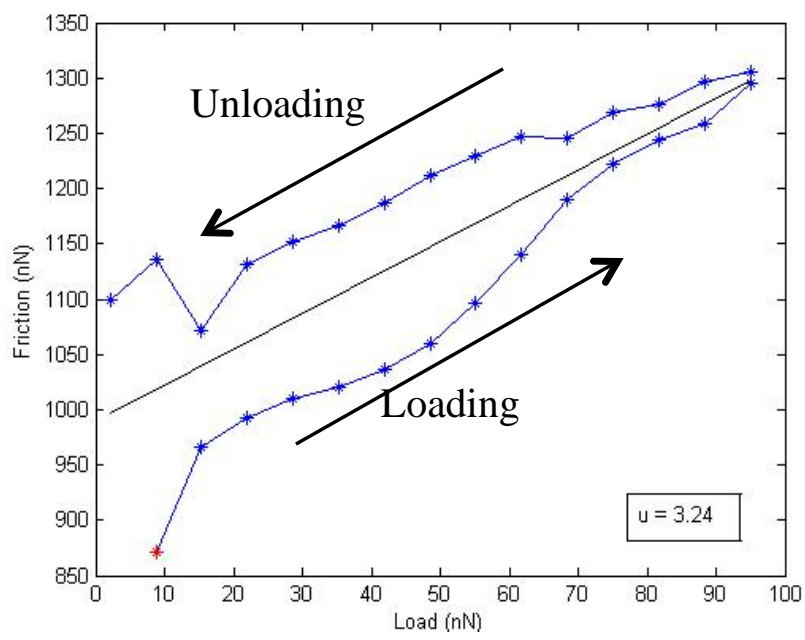

Figure 4.22: Frictional plot of bare glass substrate vs bare glass probe with varying normal load with a linear line of best fit (Amonton's law), suggesting a friction coefficient of 3.24.

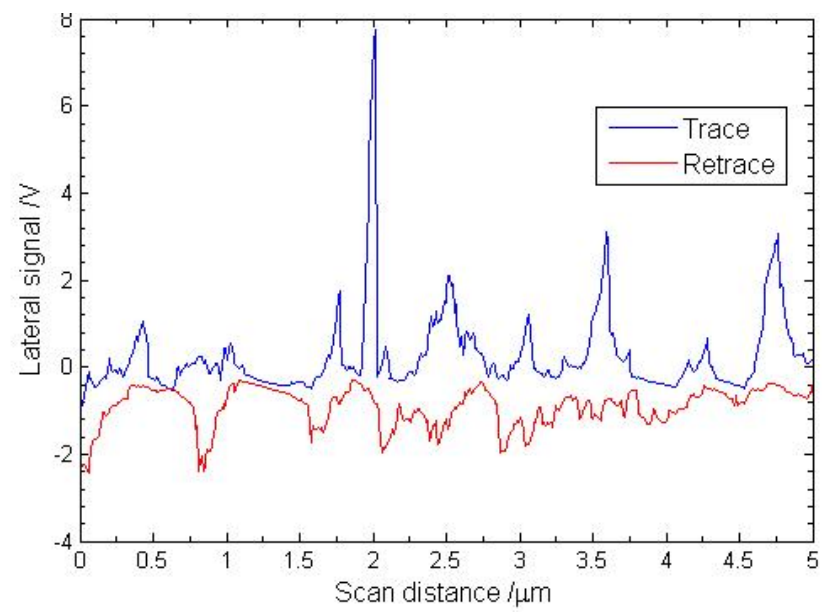

Figure 4.23: $3 \times$ seeded $\mathrm{ZnO}$ vs bare glass probe shear trace following $95 \mathrm{nN}$ of force assuming a spring constant of $0.3 \mathrm{~N} / \mathrm{m}$.

Distribution A: Approved for public release; distribution is unlimited. 


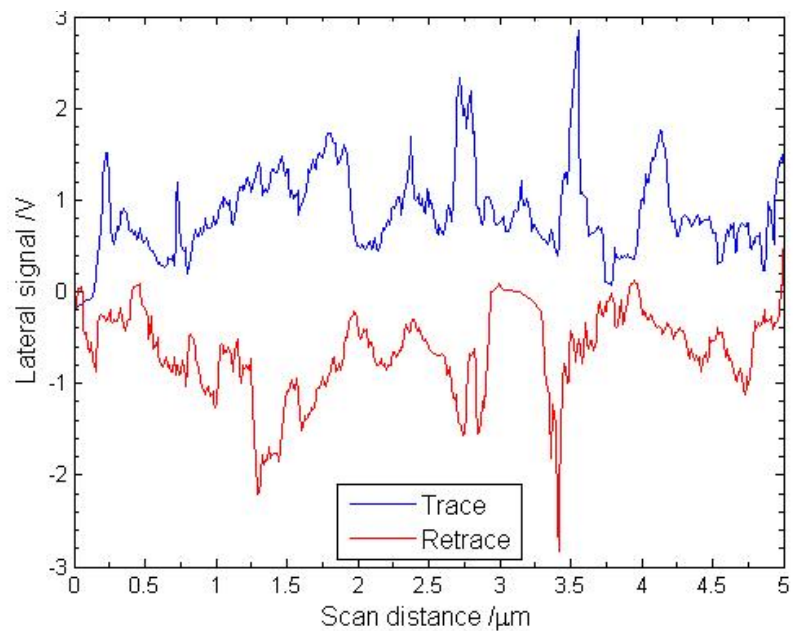

Figure 4.24: $3 \times$ seeded ZnO vs bare glass probe shear trace following $95 \mathrm{nN}$ of force assuming a spring constant of $0.3 \mathrm{~N} / \mathrm{m}$.

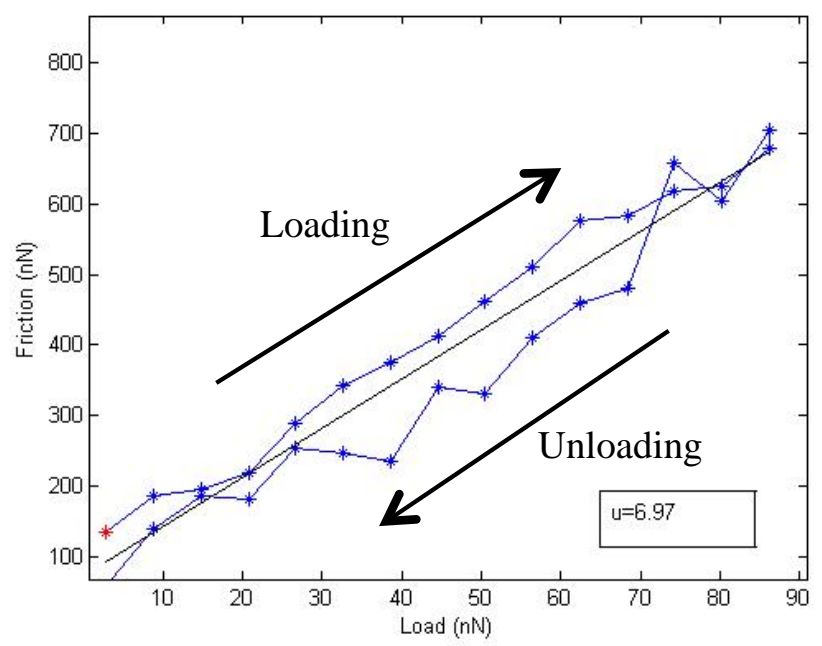

Figure 4.25: Frictional plot of a 3x seeded ZnO nanorod substrate vs bare glass probe with varying normal load with a linear line of best fit (Amontons' law), giving a friction coefficient of 6.97

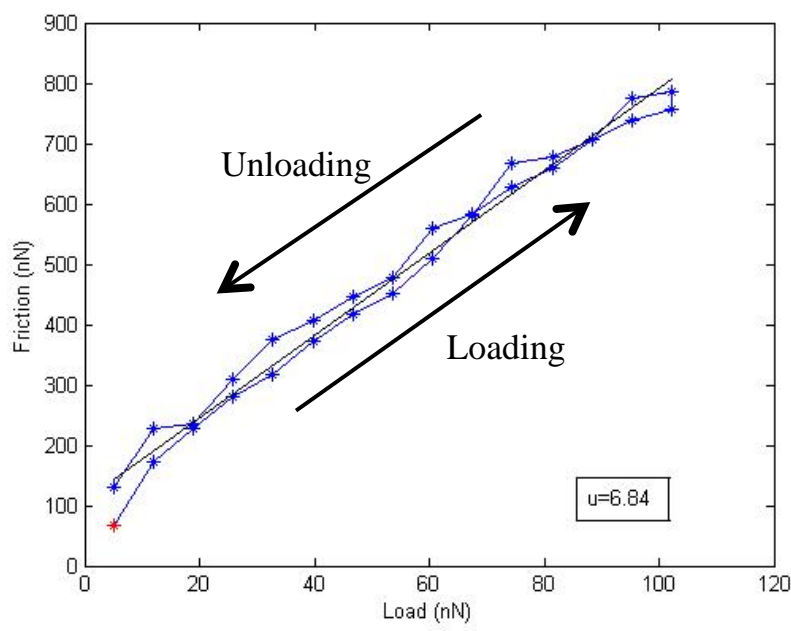

Figure 4.26: Frictional plot of a $3 \times$ seeded ZnO nanorod substrate + TEOS vs bare glass probe with varying normal load with line of best fit (Amontons' law), with a friction coefficient of 6.84.

Distribution A: Approved for public release; distribution is unlimited. 


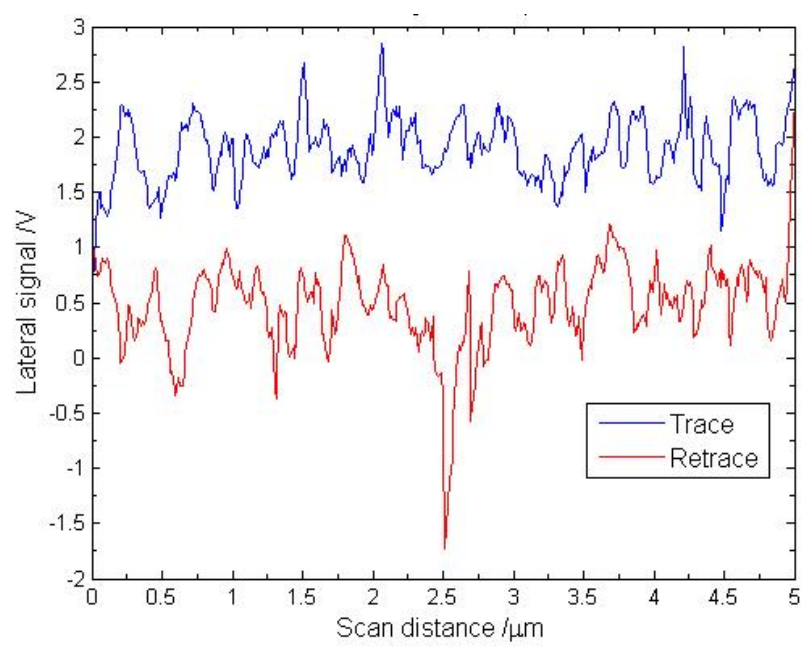

Figure 4.27: $3 \times$ seeded ZnO +TEOS vs 3x seeded ZnO +TEOS glass fibre probe shear trace (95nN assuming $0.3 \mathrm{~N} / \mathrm{m}$ )

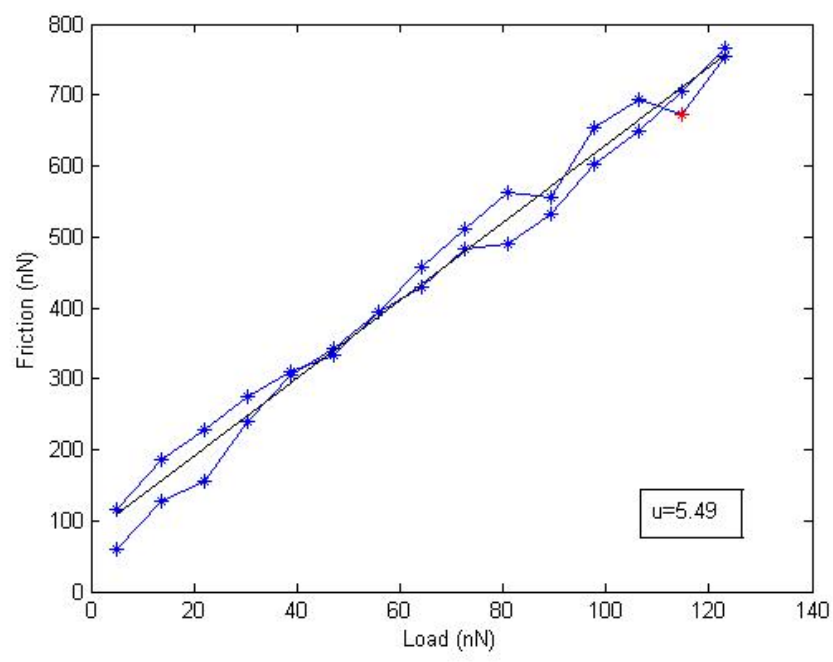

Figure 28: Frictional plot of a $3 \times$ seeded ZnO nanorod substrate + TEOS vs 3x seeded ZnO nanorod substrate + TEOS glass fibre probe with varying normal load with a linear line of best fit (Amontons' law), giving a friction coefficient of 5.49 .

\subsection{Concluding Remarks}

Morphology and alignment of $\mathrm{ZnO}$ nanorod growth through hydrothermal methods combined with an acetate seeding approach has been shown to be highly dependent on the seed density. Increasing seed density improves alignment perpendicular to the surface through the restriction of growth on non-aligned axes. Batch processed $\mathrm{ZnO}$ nanorod growth on short glass fibres has been shown to be successful; however only in areas open to nutrient supply near the perimeter of agglomerated fibres. Agglomeration of discontinuous fibres during growth limits the potential for a batch process to be utilised for composite fibre manufacture with $\mathrm{ZnO}$

Distribution A: Approved for public release; distribution is unlimited. 
nanorod attachments, and a continuous fibre growth method where fibres can be cut after growth is recommended. The method of cleaning has also shown to have had a significant effect on adhesion to the surface; with concentrated acid damaging the fibre surface causing nanorod loss. Also shown but not fully investigated is the effect on growth morphology of variable substrate surface area, something yet to be reported in current literature. Growth on a continuous glass fibre through clamping at either end is shown to be successful with extremely good coverage of nanorods. Poor heating of the individual fibril during decomposition of the zinc acetate seed is thought to be the cause of sparser rod density, whilst surface contaminants and poorer heating during growth are also thought to contribute. Tensile strength after ZnO nanorod growth followed by a TEOS coating was found to increase, with the increase likely due to the TEOS coating 'healing' surface defects and effectively increasing effective cross sectional area. AFM measurements have shown that after a TEOS coating there is reduced lateral force hysteresis, potentially indicating less surface damage from the AFM probe. Inter-digitation of nanorods against nanorods has shown a reduction in friction compared with nanorods against a bare glass surface, thought to be due to reduced rod bending caused by an overall reduction in contact area. The overriding observation from the friction measurements is that the presence of nanorods has a dramatic effect on the interfacial shear, which can be tuned by controlling the nanorod morphologies. This paves the way for future exploitation of nanorod coating as an effective strategy for controlling interfacial slip and stress in composites for enhanced ductility.

There are several areas for future research for $\mathrm{ZnO}$ nanorod application in composite materials. First and foremost is to quantify IFSS of a fibre post surface nanorod growth, either through fibre pull-out or a fibre fragmentation test. Further to this, potential for growth on an individual continuous preform is possible providing the preform has no sizing agent. Due to its crystal wurtzite structure, $\mathrm{ZnO}$ nanorods have piezoelectric behaviour and hence could be used as energy harvesters and/or as strain sensors. Carbon fibres are naturally conducting and may provide potential to carry charge produced via induced strain; providing the enticing prospect of smart fibres. Finally, patterned growth through different seeding 
techniques is possible, which may provide potential for IFSS control, controlling where facture may initiate and propagate.

\subsection{References}

1. Lakes, R., Materials with structural hierarchy. Nature, 1993. 361(6412): p. 511-515.

2. Juntaro, J., et al., Creating hierarchical structures in renewable composites by attaching bacterial cellulose onto sisal fibers. Advanced Materials, 2008. 20(16): p. 3122-3126.

3. Qian, H., et al., Carbon nanotube-based hierarchical composites: a review. Journal of Materials Chemistry, 2010. 20(23): p. 4751-4762.

4. Rho, J.-Y., L. Kuhn-Spearing, and P. Zioupos, Mechanical properties and the hierarchical structure of bone. Medical engineering \& physics, 1998. 20(2): p. 92-102.

5. Launey, M.E., M.J. Buehler, and R.O. Ritchie, On the mechanistic origins of toughness in bone. Annual Review of Materials Research, 2010. 40: p. 25-53.

6. Studart, A.R., Towards High-Performance Bioinspired Composites. Advanced Materials, 2012. 24(37): p. 5024-5044.

7. Hashin, Z., Analysis of composite materials. J. appl. Mech, 1983. 50(2): p. 481505.

8. Blumentritt, B.F., B.T. Vu, and S.L. Cooper, The mechanical properties of oriented discontinuous fiber-reinforced thermoplastics. I. Unidirectional fiber orientation. Polymer Engineering \& Science, 1974. 14(9): p. 633-640.

9. Kim, J.-K. and Y.-w. Mai, High strength, high fracture toughness fibre composites with interface control-a review. Composites Science and Technology, 1991. 41(4): p. 333-378.

10. Atkins, A., Imparting strength and toughness to brittle composites. 1974.

11. Wong, E.W., P.E. Sheehan, and C.M. Lieber, Nanobeam mechanics: elasticity, strength, and toughness of nanorods and nanotubes. Science, 1997. 277(5334): p. 1971-1975.

12. Thostenson, E.T., et al., Carbon nanotube/carbon fiber hybrid multiscale composites. Journal of Applied Physics, 2002. 91(9): p. 6034-6037.

13. Qian, H., et al., Carbon nanotube grafted carbon fibres: a study of wetting and fibre fragmentation. Composites Part A: Applied Science and Manufacturing, 2010. 41(9): p. 1107-1114.

14. Hull, D. and T. Clyne, An introduction to composite materials1996: Cambridge university press.

15. Song, J., et al., Elastic property of vertically aligned nanowires. Nano Letters, 2005. 5(10): p. 1954-1958.

16. McDowell, M.T., A.M. Leach, and K. Gall, On the elastic modulus of metallic nanowires. Nano letters, 2008. 8(11): p. 3613-3618.

17. Chen, $\mathrm{C}$., et al., Size dependence of Young's modulus in ZnO nanowires. Physical Review Letters, 2006. 96(7): p. 075505.

18. $\mathrm{Xu}, \mathrm{S}$. and Z.L. Wang, One-dimensional ZnO nanostructures: Solution growth and functional properties. Nano Research, 2011. 4(11): p. 1013-1098. 
19. Sun, B. and H. Sirringhaus, Solution-Processed Zinc Oxide Field-Effect Transistors Based on Self-Assembly of Colloidal Nanorods. Nano Letters, 2005. 5(12): p. 2408-2413.

20. Manekkathodi, A., et al., Direct Growth of Aligned Zinc Oxide Nanorods on Paper Substrates for Low-Cost Flexible Electronics. Advanced Materials, 2010. 22(36): p. 4059-4063.

21. Tsukazaki, A., et al., Repeated temperature modulation epitaxy for $p$-type doping and light-emitting diode based on ZnO. Nature materials, 2004. 4(1): p. 42-46.

22. Qin, Y., X. Wang, and Z.L. Wang, Microfibre-nanowire hybrid structure for energy scavenging. Nature, 2008. 451(7180): p. 809-813.

23. Liu, Y., et al., Growth of well-arrayed ZnO nanorods on thinned silica fiber and application for humidity sensing. Opt. Express, 2012. 20(17): p. 19404-19411.

24. Weintraub, B., Y. Wei, and Z.L. Wang, Optical Fiber/Nanowire Hybrid Structures for Efficient Three-Dimensional Dye-Sensitized Solar Cells. Angewandte Chemie, 2009. 121(47): p. 9143-9147.

25. Ni, Y., et al., Fabrication, Characterization and Properties of Flowerlike ZnS-ZnO Heterogeneous Microstructures Built Up by ZnS-Particle-Strewn ZnO Microrods. The Journal of Physical Chemistry C, 2008. 112(22): p. 8200-8205.

26. Wang, Z., et al., Aqueous solution fabrication of large-scale arrayed obelisklike zinc oxide nanorods with high efficiency. Journal of Solid State Chemistry, 2004. 177(6): p. 2144-2149.

27. Xu, S., et al., Optimizing and improving the growth quality of ZnO nanowire arrays guided by statistical design of experiments. ACS nano, 2009. 3(7): p. 1803-1812.

28. Vayssieres, L., Growth of Arrayed Nanorods and Nanowires of ZnO from Aqueous Solutions. Advanced Materials, 2003. 15(5): p. 464-466.

29. Allen, R.J.A., et al., Investigations into the Potential of Constructing Aligned Carbon Nanotube Composite Materials through Additive Layer Manufacture. 2013.

30. Thomason, J., et al., Influence of fibre length and concentration on the properties of glass fibre-reinforced polypropylene: Part 3. Strength and strain at failure. Composites Part A: Applied Science and Manufacturing, 1996. 27(11): p. 1075-1084.

31. Fu, S.-Y., et al., Tensile properties of short-glass-fiber-and short-carbon-fiberreinforced polypropylene composites. Composites Part A: Applied Science and Manufacturing, 2000. 31(10): p. 1117-1125.

32. Qian, H., et al., Hierarchical composites reinforced with carbon nanotube grafted fibers: the potential assessed at the single fiber level. Chemistry of Materials, 2008. 20(5): p. 1862-1869.

33. García, E.J., A.J. Hart, and B.L. Wardle, Long carbon nanotubes grown on the surface of fibers for hybrid composites. AIAA journal, 2008. 46(6): p. 14051412.

34. Wicks, S.S., R.G. de Villoria, and B.L. Wardle, Interlaminar and intralaminar reinforcement of composite laminates with aligned carbon nanotubes. Composites Science and Technology, 2010. 70(1): p. 20-28. 
35. Mathur, R., S. Chatterjee, and B. Singh, Growth of carbon nanotubes on carbon fibre substrates to produce hybrid/phenolic composites with improved mechanical properties. Composites Science and Technology, 2008. 68(7): p. 1608-1615.

36. Gibson, R.F., A review of recent research on mechanics of multifunctional composite materials and structures. Composite Structures, 2010. 92(12): p. 2793-2810.

37. Garcia, E.J., et al., Fabrication and multifunctional properties of a hybrid laminate with aligned carbon nanotubes grown< i> In Situ</i>. Composites Science and Technology, 2008. 68(9): p. 2034-2041.

38. Rahmanian, S., et al., Carbon and glass hierarchical fibers: Influence of carbon nanotubes on tensile, flexural and impact properties of short fiber reinforced composites. Materials \& Design, 2013. 43(0): p. 10-16.

39. Kashif, M., et al., Effect of different seed solutions on the morphology and electrooptical properties of ZnO nanorods. J. Nanomaterials, 2012. 2012: p. 106-106.

40. Guo, M., P. Diao, and S. Cai, Hydrothermal growth of well-aligned ZnO nanorod arrays: Dependence of morphology and alignment ordering upon preparing conditions. Journal of Solid State Chemistry, 2005. 178(6): p. 18641873.

41. Fuge, G.M., T.M.S. Holmes, and M.N.R. Ashfold, Ultrathin aligned ZnO nanorod arrays grown by a novel diffusive pulsed laser deposition method. Chemical Physics Letters, 2009. 479(1-3): p. 125-127.

42. Umar, A., et al., Growth of Highly c-Axis-Oriented ZnO Nanorods on ZnO/Glass Substrate: Growth Mechanism, Structural, and Optical Properties. The Journal of Physical Chemistry C, 2009. 113(33): p. 14715-14720.

43. Xu, C.X., et al., Zinc oxide nanowires and nanorods fabricated by vapourphase transport at low temperature. Nanotechnology, 2004. 15(7): p. 839.

44. Li, J.Y., et al., Fabrication of zinc oxide nanorods. Journal of Crystal Growth, 2001. 233(1-2): p. 5-7.

45. Razak, A.F.A., et al., Effect of annealing on structural and optical Properties of ZnO thin films by sol gel technique. Chalcogenide Letters, 2011. 8(9): p. 511519.

46. Greene, L.E., et al., General route to vertical ZnO nanowire arrays using textured $\mathrm{ZnO}$ seeds. Nano Letters, 2005. 5(7): p. 1231-1236.

47. Wang, D., et al., ZnO nanorod array polydimethylsiloxane composite solid phase micro-extraction fiber coating: fabrication and extraction capability. Analyst, 2012. 137(2): p. 476-480.

48. Zhu, R., et al., Uniform Zinc Oxide Nanowire Arrays Grown on Nonepitaxial Surface with General Orientation Control. Nano Letters, 2013. 13(11): p. 5171-5176.

49. Zhang, T., et al., Site-specific nucleation and growth kinetics in hierarchical nanosyntheses of branched $\mathrm{ZnO}$ crystallites. Journal of the American Chemical Society, 2006. 128(33): p. 10960-10968.

50. Norma, A., C 1557-03. Standard Test Method for Tensile Strength and Young's Modulus of Fibers. 
51. Thormann, E., et al., Amontonian Friction Induced by Flexible Surface Features on Microstructured Silicon. ACS Applied Materials \& Interfaces, 2011. 3(9): p. 3432-3439.

52. Sader, J.E., J.W.M. Chon, and P. Mulvaney, Calibration of rectangular atomic force microscope cantilevers. Review of Scientific Instruments, 1999. 70(10): p. 3967-3969.

53. Green, C.P., et al., Normal and torsional spring constants of atomic force microscope cantilevers. Review of Scientific Instruments, 2004. 75(6): p. 1988-1996.

54. Pilkington, G.A., et al., Amontonian frictional behaviour of nanostructured surfaces. Physical Chemistry Chemical Physics, 2011. 13(20): p. 9318-9326.

55. Bulla, D.A.P. and N.I. Morimoto, Deposition of thick TEOS PECVD silicon oxide layers for integrated optical waveguide applications. Thin Solid Films, 1998. 334(1-2): p. 60-64.

56. Cannas, A., Fracture Mechanics and Failure Analysis of Hollow Shaped Fibre GFRP Composites, in ACCIS, Department of Aerospace Engineering2008, Bristol.

57. Quignon, B., et al., Sustained Frictional Instabilities on Nanodomed Surfaces: Stick-Slip Amplitude Coefficient. ACS nano, 2013. 


\section{Ultrasonic alignment of micron length scale reinforcement}

\subsection{Introduction}

To this day, the majority of ultrasonic devices have been developed for applications in biological and life sciences. Consequently, designs are generally tailored to, and optimised for the operation in an aquaeous environment. In this study, the primary objective is to develop a design that allows for the rapid and repeatable fabrication of thin layers of composite material at the micron length scale; a secondary motivation is founded on the desire to maximise the range of fibre architectures that can be formed using a particular ultrasonic device. The final design is to be devised taking a counter-propagating wave approach, as this method is considered to be the least sensitive to changes in the fluid chamber's resonant frequency when large quantities of particles (at the micron length scale) are present. Moreover, samples are to be sufficiently large to allow for macroscopic mechanical characterisation to be carried out.

\subsection{Device design: Two-element array}

The two-element array is the simplest form of counter-propagating wave device and was used extensively in this study for the manufacture of thin composite samples with unidirectional reinforcement. The device is shown schematically in Figure 5.1 below. 
a.

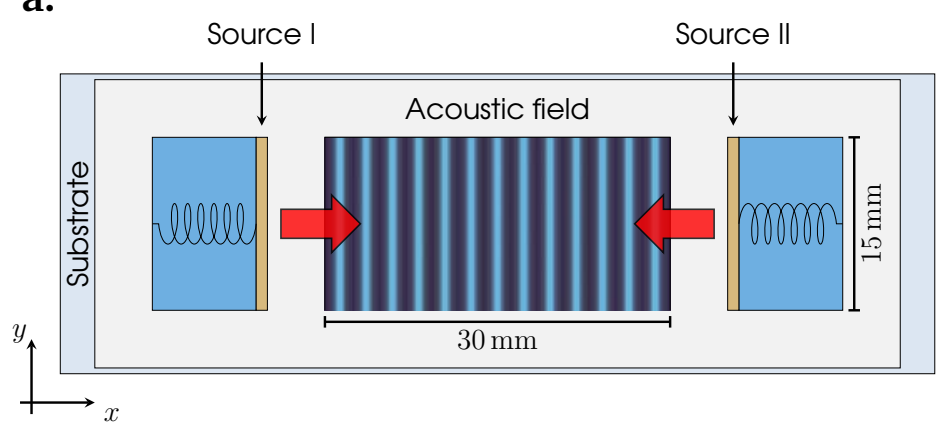

b.

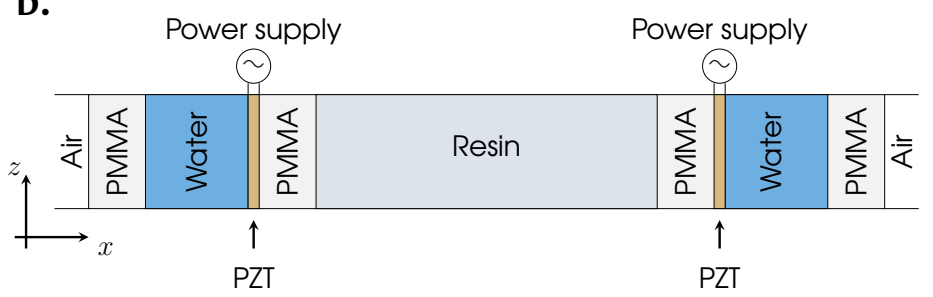

Figure 5.1: Third generation two-element array. (a) A standing wave acoustic field is formed inside a central fluid volume, by interference of two counter-propagating traveling waves. The PZT transducers are submerged in water for cooling purposes, and held in place by small compression springs. (b) A device representation by material layers: air (1), PMMA (5 mm), H2O (9.025 mm), PZT (0.975 mm), PMMA $(5 \mathrm{~mm})$, resin $(30 \mathrm{~mm})$, PMMA $(5 \mathrm{~mm})$, PZT $(0.975 \mathrm{~mm})$, H2O $(9.025 \mathrm{~mm})$, PMMA (5mm), air (1).

As shown in Figure 5.1, two $0.975 \mathrm{~mm} \times 15 \mathrm{~mm} \times 2 \mathrm{~mm}$ soft-doped PZT (Noliac group, NCE51) elements were placed on opposite ends of the device, each separated from the central cavity by a sacrificial PMMA boundary. On either side of the device, an additional water filled chamber served the purpose of a necessary heat sink at high driving voltages. The PMMA frame was mounted on a glass substrate using adhesive tape (tesa 64621-00007-01). For both acoustic drivers to be more easily recycled, a small compression spring secured each of them against the adjoining PMMA boundary; a significantly increased composite production efficiency was the immediate result. With the central cavity now measuring $30 \mathrm{~mm} \times 15 \mathrm{~mm} \times$ $2 \mathrm{~mm}$ (a size suitably large to manufacture test specimens for mechanical 
characterization), the driving voltage was raised to $80 \mathrm{~V}_{\mathrm{pp}}$. An operation at relatively low voltages is desirable primarily to minimise the effects of acoustic streaming inside the central cavity. In the $x-y$ plane the sample size was constrained to the dimensions of the device cavity, the thickness (z-direction) could be controlled by varying the amount of resin added to the chamber.

The composite assembly time is critically dependent on viscosity, with the fluid drag constituting the dominant resistive force to any particle motion. A low viscosity resin is thus generally preferred. For short glass fibres $(r \leq 25 \mu \mathrm{m})$ dispersed in water $\left(\eta_{\text {water }} \approx 0.8 \times 10-3\right.$ Pa s), full assembly is experimentally achieved in less than $100 \mathrm{~ms}$, with particles traveling to pressure nodes. Given a linear dependency of the drag force on viscosity, an equivalent assembly time of $950 \mathrm{~ms}$ is calculated for fibres suspended in the resin.

\subsection{Materials}

A blend of fibre composite material used was based on the aromatic dimethacrylate monomer 2,2-bis[p-(2-hydroxy-3methacryloxypropoxy)phenyl]propane (BisGMA), a foundation commonly used for dental composite applications. To lower the viscosity of the bulky BisGMA monomer (from $\eta_{\text {BisGMA }} \approx 800 \mathrm{~Pa} s$ to $\eta_{\text {resin }} \approx 0.76 \mathrm{~Pa} \mathrm{~s}$ [24]), it was mixed with the comonomer triethylene glycol dimethacrylate (TEGDMA) in a ratio of $1: 1$ by weight. $0.2 \mathrm{wt} \%$ of camphorquinone (CQ) and $0.8 \mathrm{wt} \%$ of Ethyl 4- (dimethylamino)benzoate (EDB) were also added to achieve radical photo-polymerisation at a wavelength of $460 \mathrm{~nm}$. Table 5.1 illustrates the molecular structures of each of these components. Complete ploymerisation was reached following exposure to a $900 \mathrm{~mW}$ light-emitting diode (LED) for approximately $40 \mathrm{~s}$. 
Table 5.1: Molecular structures of BisGMA, TEGDMA, EDB, and CQ.

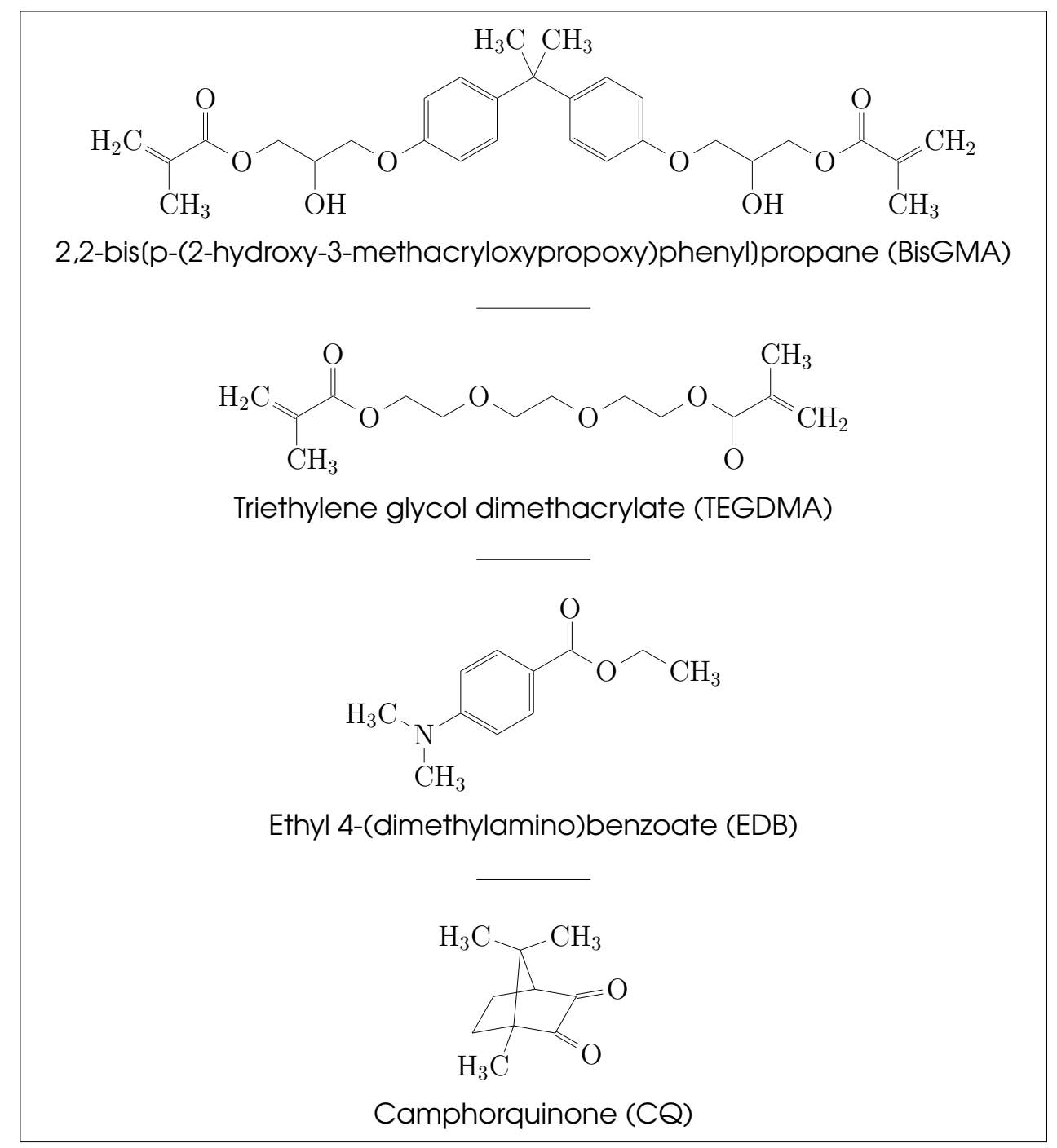

Commercially available, milled glass fibres (Lanxess, MF7904) were used as reinforcement, with a nominal length of $50 \mu \mathrm{m}$ and approximately $14 \mu \mathrm{m}$ in diameter.

\subsection{Fabrication of epoxy test specimens}

The fabrication process for the ultrasonically assembled samples, is shown diagrammatically in Figure 5.2. Firstly, some dry fibre reinforcements were distributed on the substrate. About $0.8 \mathrm{ml}$ of resin were then added, before the two phases were mixed to give an even dispersion of glass particles on the bottom surface. An ultrasonic field was applied to drive the fibres to the nodal positions of the resulting standing wave. Given a driving frequency of $v=2.01 \mathrm{MHz}$ and assuming a speed of sound, $\mathrm{c} 1=1480 \mathrm{~m} \mathrm{~s}-1$ in the fluid, lines of fibres are predicted to form at

\section{Distribution A: Approved for public release; distribution is unlimited.}


a separation of $\lambda / 2=368 \mu \mathrm{m}$; experimental measures find this separation to be in the region of $350 \mu \mathrm{m}$ to $380 \mu \mathrm{m}$. Prior to polymerisation initiation, reinforcement entities were allowed approximately $60 \mathrm{~s}$ to arrange and orient themselves under the influence of ultrasound. Finally, the resin was exposed to a blue light LED and cured in $40 \mathrm{~s}$. The microstructure of the resulting samples is shown in Figures 5.3 (a) and (e).

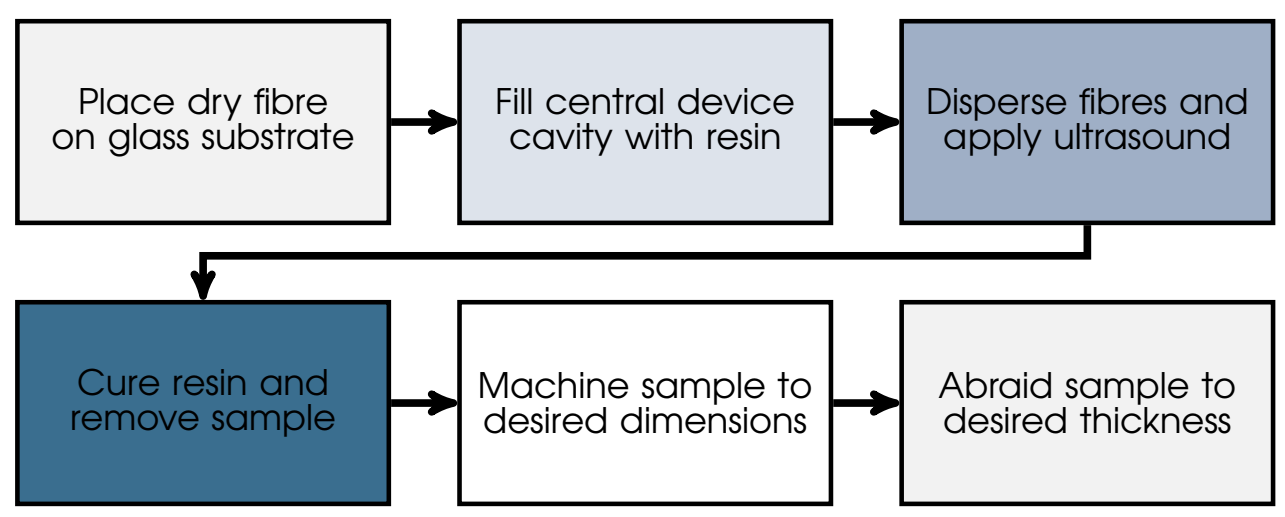

Figure 5.2: Sample fabrication process. 

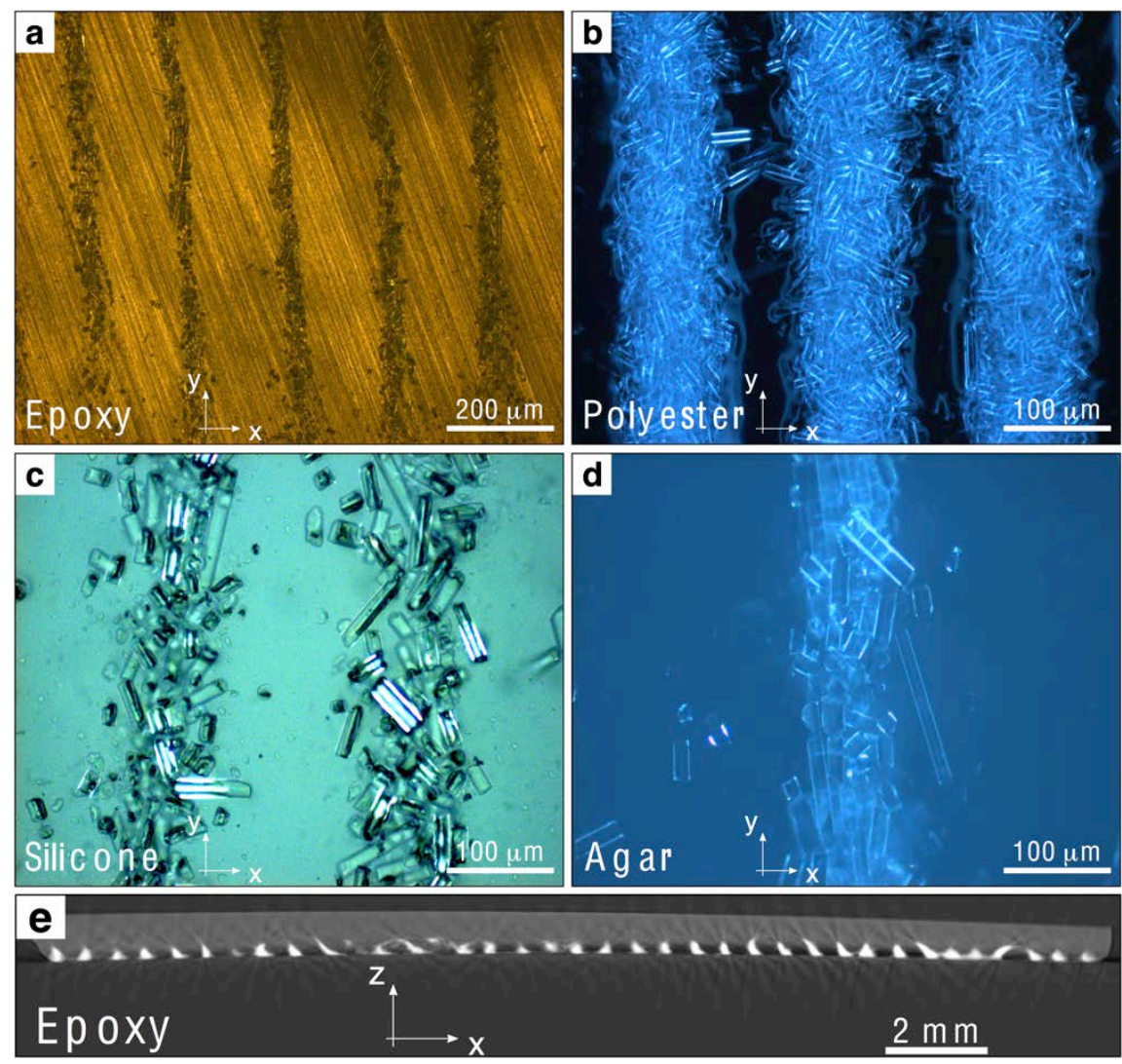

Figure 5.3: Ultrasonic assembly of glass particles into lines of unidirectional reinforcement in (a) epoxy ( $\left.c=1510 \mathrm{~m} \mathrm{~s}^{-1} \pm 2.8 \%\right)$, (b) polyester $\left(c=1347 \mathrm{~m} \mathrm{~s}^{-1} \pm 2.5\right.$ $\%)$, (c) silicone $\left(c=1070 \mathrm{~ms}^{-1} \pm 4.4 \%\right)$, (d) agar $\left(c=1513 \mathrm{~ms}^{-1} \pm 2.9 \%\right)$. Examples are shown at different magnifications and for varying fibre volume fractions. A typical sample cross-section (e) is presented in the form of a x-ray micro-tomography image. Fibre lines extend along the $y$-direction and are separated in the $x$-direction by $a$ theoretical distance of $\lambda / 2=c / 2 f$, where $c$ is the speed of sound in the matrix material and $f$ is the driving frequency.

Following the removal of the acoustic drivers and the substrate, two identical $13.6 \mathrm{~mm} \times 13.6 \mathrm{~mm}$ test pieces were cut from the original sample using a high precision carbon dioxide ( $\mathrm{CO} 2)$ laser. Across samples, the fibre volume fraction was estimated to be $\mathrm{Vf}=9.0 \pm 2.0 \%$, by $\mu \mathrm{CT}$ analysis; for each pair of test pieces, $\mathrm{Vf}$ was assumed to be the same. By means of the above procedure a true comparison of mechanical test results could be established for each pair of test pieces. An analysis 
between samples also serves to quantify general manufacture consistency, to estimate average strength and stiffness values, and to determine failure mechanisms. The slight meniscus formed by excess resin on top of each specimen was removed by abrading all specimens to a uniform thickness of $0.50 \pm 0.05 \mathrm{~mm}$. To allow for better gripping of the test pieces during mechanical testing (in tension), end tabs - as depicted in Figure 5.4 - were attached to the specimens. A set each of randomly dispersed fibre samples and neat resin specimens were prepared in the same manner but no ultrasonic energy was applied in these cases.

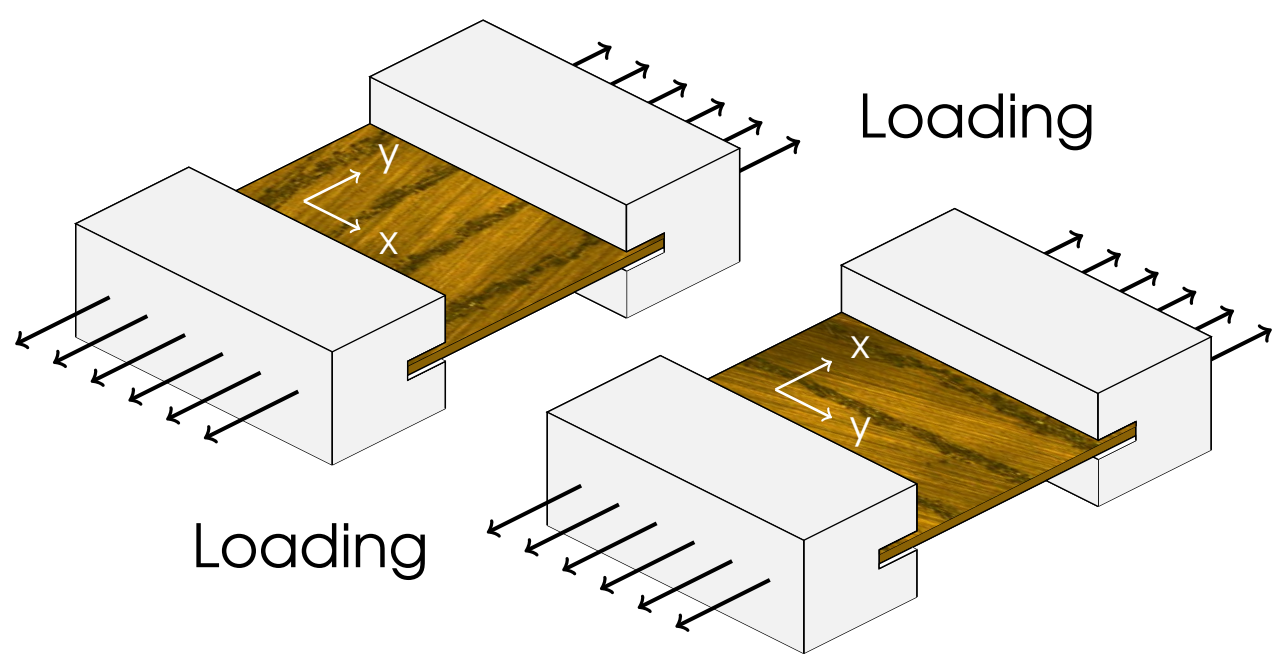

Figure 5.4: Schematic illustration of the specimen geometry for mechanical characterisation. End tabs were adhered to each pair of test specimens. In one case end tabs were attached parallel to the direction of fibre reinforcement, in the other transversely oriented.

\subsection{Mechanical Characterisation}

The samples were mechanically tested in tension at a displacement controlled crosshead speed of $0.10 \mathrm{~mm}$ min-1. The results are summarised in the form of stress vs. strain plots, shown in Figure 5.5 with the average failure and stiffness results summarized in Table 5.2 . 
a.

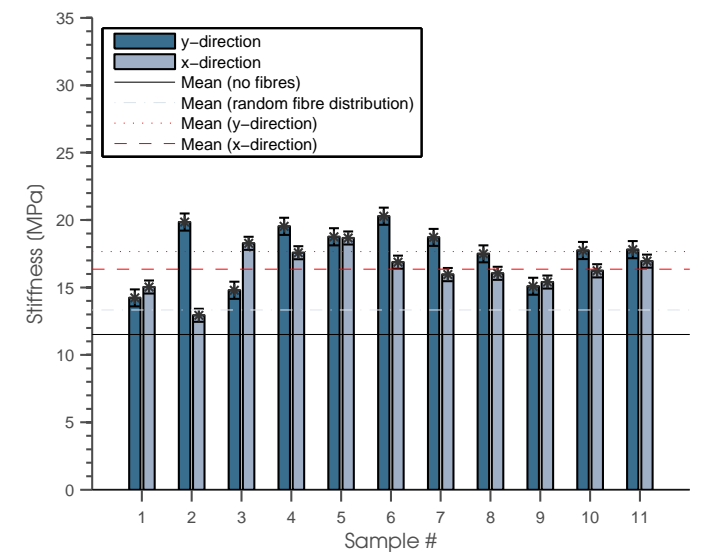

b.

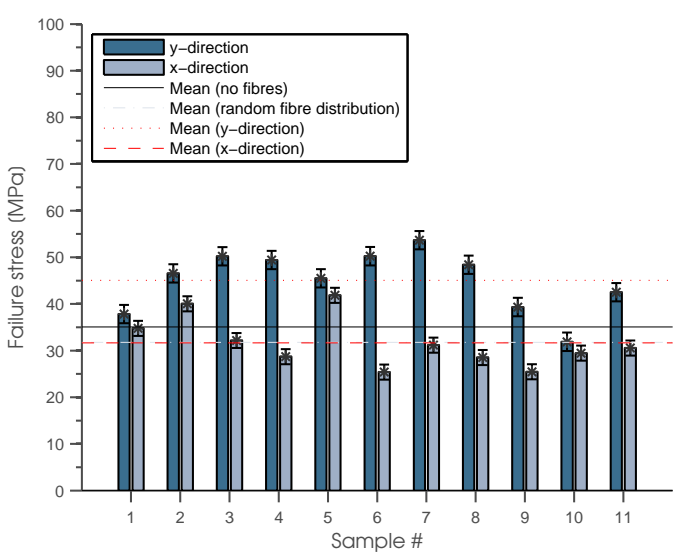

Figure 5.5: A comparison of (a) stiffness, and (b) failure stress between samples undergoing tensile loading in the $x$-and in the $y$-direction. For both cases, the mean and standard error are indicated as a reference.

Table 5.2: Average results for strain to failure, stress to failure, and stiffness. Values are presented for four different sample types, and the associate standard error is given in all cases.

\begin{tabular}{lccc}
\hline Sample type & $\begin{array}{c}\text { Failure strain, } \epsilon \\
{[\%]}\end{array}$ & $\begin{array}{c}\text { Failure stress, } \sigma \\
{[\mathrm{MPa}]}\end{array}$ & $\begin{array}{c}\text { Stiffness, } E \\
{[\mathrm{MPa}]}\end{array}$ \\
\hline No fibres & $4.55 \pm 0.41$ & $35.07 \pm 1.24$ & $11.51 \pm 0.61$ \\
Random fibre distribution & $2.63 \pm 0.17$ & $31.76 \pm 1.85$ & $13.33 \pm 0.61$ \\
$x$-direction & $2.04 \pm 0.13$ & $31.64 \pm 1.61$ & $16.36 \pm 0.48$ \\
$y$-direction & $3.00 \pm 0.15$ & $45.05 \pm 1.97$ & $17.66 \pm 0.63$
\end{tabular}

An average stiffness of $16.36 \pm 0.48 \mathrm{MPa}$ was recorded for transversely strained samples; in comparison, specimens loaded in the direction of fibre reinforcement consistently showed superior mechanical performance with an average stiffness of $17.66 \pm 0.63 \mathrm{MPa}$. The Young's modulus of samples without reinforcement and randomly oriented reinforcement were obtained as $11.51 \pm$ $0.61 \mathrm{MPa}$ and $13.33 \pm 0.61 \mathrm{MPa}$, respectively. It is thus evident that improved structural properties and anisotropy have been achieved. On comparison between samples with randomly oriented reinforcements and ultrasonically assembled samples strained transversely, it should be noted that the latter appear to provide greater stiffness. It is believed that this is due to the fact that within the assembled 
lines, fibres are in close proximity allowing interaction and load transfer, which results in increased stiffness. Within the random samples the fibres are dispersed and cannot interact.

As is clear from both Figure 5.5 and Table 5.2, the matrix itself exhibits a more ductile mechanical behaviour with strains to failure of up to $5.55 \pm 0.41 \%$; on the addition of fibre reinforcement, a more brittle failure mechanism is observed with failure generally occurring in a region of 2 to $3 \%$ strain.

For an estimation of the expected composite stiffness, $E_{y}$, the basic rule of mixtures (assuming continuous fibre reinforcement) was applied:

$$
E_{y}=V_{f} E_{f}+\left(1-V_{f}\right) E_{m}
$$

Here, $E_{m}$, and $E_{f}$ are the Young's modulus of the matrix and the fibres, respectively. Across all samples, an average $\mathrm{E}_{\mathrm{y}}=18.97 \pm 0.57 \mathrm{MPa}$ was so calculated, taking $V_{f}=9.0 \pm 2.0 \%$ as previously obtained from $\mu \mathrm{CT}$ analysis (Figure $5.3(\mathrm{e})$ ), which is in good agreement with that measured experimentally.

\subsection{Concluding Remarks}

This study details (1) the mathematical foundation for defining the dynamic evolution of an optically suspended particle (as detailed fully in Appendix A), and (2) the experimental evaluation of an ultrasonic device for the manufacture of short fibre composites. These are discussed in-turn below.

The mathematical foundation illustrated that individual control can be obtained for every reinforcement entity, thus allowing highly complex fibre constructs to be formed. While particles cannot be treated independently in ultrasonic fields, these appear to be more viable for applications on the macroscale. A study using water as the host medium, indicated good agreement between the mathematical model and the experimental studies. However, it should be noted that no exact prediction can be made of the total number of particles that are drawn 
towards a single node but, to gain some indication, it may be possible to estimate the volume of low pressure and compare this with the particles' dimensions. In the case of larger particles, some form of 'bridging' across multiple nodes is to be expected, most likely following the path of least resistance.

In the second half of the study, an ultrasonic particle manipulation technique was developed and employed to manufacture thin, single layers of short glass fibre reinforced polymer composites. To achieve this, a new type of ultrasonic device was developed, separating the acoustic system from the resin cavity to allow for the easy manufacture of multiple samples. Furthermore, an effective method has been outlined to manufacture and mechanically characterise these thin laminar two-phase materials. With an $8 \%$ difference in stiffness between the two principal directions, anisotropy was demonstrated for unidirectionally reinforced discontinuous fibre composites under uniaxial tensile loading. A $43 \%$ improvement in strength could be observed for samples tested along the direction of fibre reinforcement, over those strained in the $x$-direction, despite the relatively low volume percentage of the reinforcement phase.

\subsection{References}

[1] P. Fratzl, Cellulose and collagen: from fibres to tissues, Current Opinion in Colloid \& Interface Science 8 (2003) 32 - 39.

[2] P.Fratzl, R. Weinkamer, Nature's hierarchical materials, Progress in Materials Science 52 (2007) $1263-1334$.

[3] G. Otieno, A. Koos, F. Dillon, A. Wallwork, N. Grobert, R. Todd, Processing and properties of aligned multi-walled carbon nanotube/aluminoborosilicate glass composites made by solgel pro- cessing, Carbon 48 (2010) $2212-2217$.

[4] W. Wang, P. Ciselli, E. Kuznetsov, T. Peijs, A. Bar- ber, Effective reinforcement in carbon nanotubepolymer composites, Philosophical transactions. Series A, Mathematical, physical, and engineering sciences 366 (2008) $1613-1626$.

[5] L. Jin, C. Bower, O. Zhou, Alignment of carbon nanotubes in a polymer matrix by mechanical stretching, Applied Physics Letters 73 (1998) 1197 - 1199.

[6] A. Ashkin, J. Dziedzic, J. Bjorkholm, S. Chu, Observation of a single-beam gradient force optical trap for dielectric particles, Opt. Lett. 11 (1986) 288 290.

[7] R. Gauthier, Theoretical investigation of the optical trapping force and torque on cylindrical micro- objects, J. Opt. Soc. Am. B 14 (1997) 3323 - 3333. 
[8] R. Gauthier, Trapping model for the low-index ring- shaped micro-object in a focused, lowest-order gaussian laser-beam profile, J. Opt. Soc. Am. B 14 (1997) $782-789$.

[9] R.Gauthier, S.Wallace, Optical levitation of spheres: analytical development and numerical computations of the force equations, J. Opt. Soc. Am. B 12 (1995) $1680-1686$.

[10] R. Gauthier, M. Ashman, C. Grover, Experimental confirmation of the opticaltrapping properties of cylindrical objects, Appl. Opt. 38 (1999) $4861-4869$.

[11] W. Wright, G. Sonek, M. Berns, Parametric study of the forces on microspheres held by optical tweezers, Appl. Opt. 33 (1994) $1735-1748$.

[12] T.Nieminen, H.Rubinsztein-Dunlop, N.Heckenberg, Calculation and optical measurement of laser trapping forces on non-spherical particles, Journal of Quantitative Spectroscopy and Radiative Transfer 70 (2001) 627-637.

[13] T. Grzegorczyk, B. Kemp, J. Kong, Trapping and binding of an arbitrary number of cylindrical particles in an in-plane electromagnetic field, J. Opt. Soc. Am. A 23 (2006) $2324-2330$.

[14] K. Neuman, S. Block, Optical trapping, Review of Scientific Instruments 75 (2004) $2787-2809$.

[15] G. Whyte, G. Gibson, J. Leach, M. Padgett, D. Robert, M. Miles, An optical trapped microhand for manipulating micron-sized objects, Opt. Express 14 (2006) $12497-12502$.

[16] G.Gibson,D.Carberry,G.Whyte,J.Leach,J.Courtial, J. Jackson, D. Robert, M. Miles, M. Padgett, Holographic assembly workstation for optical manipulation, Journal of Optics A: Pure and Applied Op- tics 10 (2008) 044009. 


\section{Research Summary and Future Work}

This report details three specific investigations into the generation of both complex hierarchical fibre architecture and controlled ultrasonic field effect techniques (at different length scales) for additive layer manufacturing of biologically inspired short fibre reinforced composites.

The work has demonstrated the successful formulation of Zinc oxide ( $\mathrm{ZnO}$ ) nanorods grown in aqueous solutions onto the surface of E-glass short fibres (approximately $3 \mathrm{~mm}$ in length), and a continuous borosilicate glass fibre $60 \mathrm{~mm}$ in length. The nanorods do not compromise the mechanical properties of the fibres and furthermore the TEOS coating could 'heal' defects and cause a slight increase in fibre cross sectional area. In the second half of the study, both theoretical analysis and experimental development were used to evaluate the potential of field gradient technologies at generating (1) micron length scale aligned reinforcement and (2) nano length scale aligned composite materials, with regard to rapid prototyping manufacture of complex fibre constructs.

To achieve this, a new type of ultrasonic device was developed, separating the acoustic system from the resin cavity to allow for the easy manufacture of multiple samples. Furthermore, an effective method has been outlined to manufacture and mechanically characterise the micron length scale reinforced thin laminar composites. With an $8 \%$ difference in stiffness between the two principal directions, anisotropy was demonstrated for unidirectionally reinforced discontinuous fibre composites under uniaxial tensile loading. A $43 \%$ improvement in strength was observed for samples tested parallel to the direction of the fibre reinforcement over those strained normal to the fibre direction, despite the relatively low volume percentage of the reinforcement phase. This technique shows great potential for the low cost instantaneous alignment of structural reinforcement to generate the lightweight high performance structures required for future military requirements.

The orientation and distribution of reinforcing entities in engineering composites is key to enabling structural efficiency, yet the architecture remains 
simplistic when compared to the distinctive and unique hierarchies found in Nature. These biological 'composite' materials achieve such configurations by accurately controlling the orientation of anisotropic nano- and micro-sized 'building blocks', thereby reinforcing the material in specific directions to carry the multidirectional external loads at different length scales. Capturing the design principles underlying the exquisite architecture of such biological materials will overcome many of the mechanical limitations of current engineering composites.

This research has investigated one possible route to the realisation of advanced structural metamaterials (i.e. materials that today do not exist in either engineered or biological forms) with controlled orientation as a 'bottom-up' manufacturing technique for integration within AM. This study has clearly illustrated the potential for controlled instantaneous alignment of structural reinforcement. Through the application of novel multifunctional fibres and matrix materials with field effect techniques (ultrasonic/magnetic) new structural designs will be realised that are hierarchical, multi-material, multi-functional, and multi-scale offering reduced weight, improved reliability and affordability.

To achieve this 'future', active advanced multifunctional materials, exhibiting programmed intelligence in complex 3D architectures, should, and could, be developed via creative manufacturing. This will require (1) new hierarchical fibrous metamaterials, (2) the on-going development of field effect techniques and (3) the development of novel smart matrices. One possible research programme to develop these materials of the future is illustrated in Figure 6.1. 

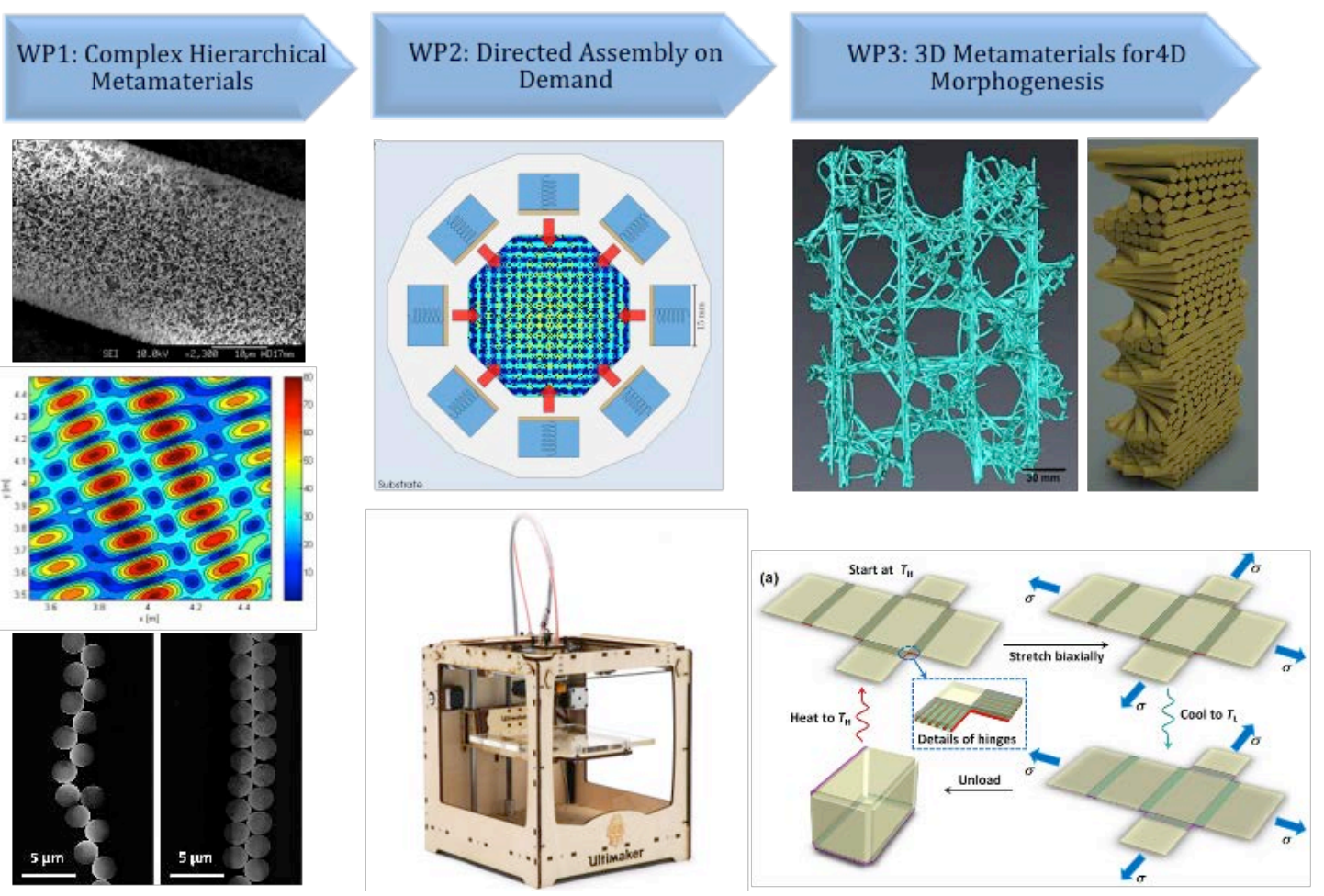

Figure 6.1: Overview of future research concepts: Left Column- WP1: Top, Hierarchical reinforcement (ZnO nanorods on glass fibres); Mid, ultrasonic field effect prediction; Lower, Janus particles magnetically aligned; Centre Column- WP2: Top, acoustic assembly pattern prediction for radial transducers; Lower, Ultimaker desktop 3D printer; Right Column- WP3: Top, biological functionally graded architecture; Lower, self-folding architecture.

These new modes of assembly, i.e. manufacturing as a growth process, will rely on smarter materials, not machines of increasing complexity. Figure 6.1 shows the three key overriding elements of a future programme directed towards the development of metamaterials for 4D morphogenesis; namely synthetic processing routes based on 'complex hierarchical metamaterials', 'mechanically coupled fieldeffect assembly' and '3D Additive Manufacturing'. It is proposed that through the use of planar patterning of microparticles and/or nanoparticles, it will be possible to achieve programmable and reconfigurable architectures through the field effect formation and control of 'acoustic or magnetic pixels'. If successfully developed and exploited, this would generate a unique method for reducing lead-times (through 
rapid design to production) and add significant value across all engineering disciplines through smarter materials and structures, not just smarter machines.

Distribution A: Approved for public release; distribution is unlimited. 


\section{Appendix A: Optical levitation of cylindrical parameters}

A detailed derivation of the force equations, mathematically describing the behaviour of small levitated cylinders in a beam of light, is presented below. Whilst closely following theoretical interpretations by Gauthier and Wallace [9], the present approach embraces some added geometrical complexity. The model is considered valid for all systems satisfying the condition $d>10 \lambda_{0}$, where $d$ is the diameter of the particle and 760 the wavelength of electromagnetic radiation in free space. A ray diagram defining any geometrical parameters is presented in Figure A.1. In addition, Figure A.2 visually presents the constant force fields that surround the levitated particle.

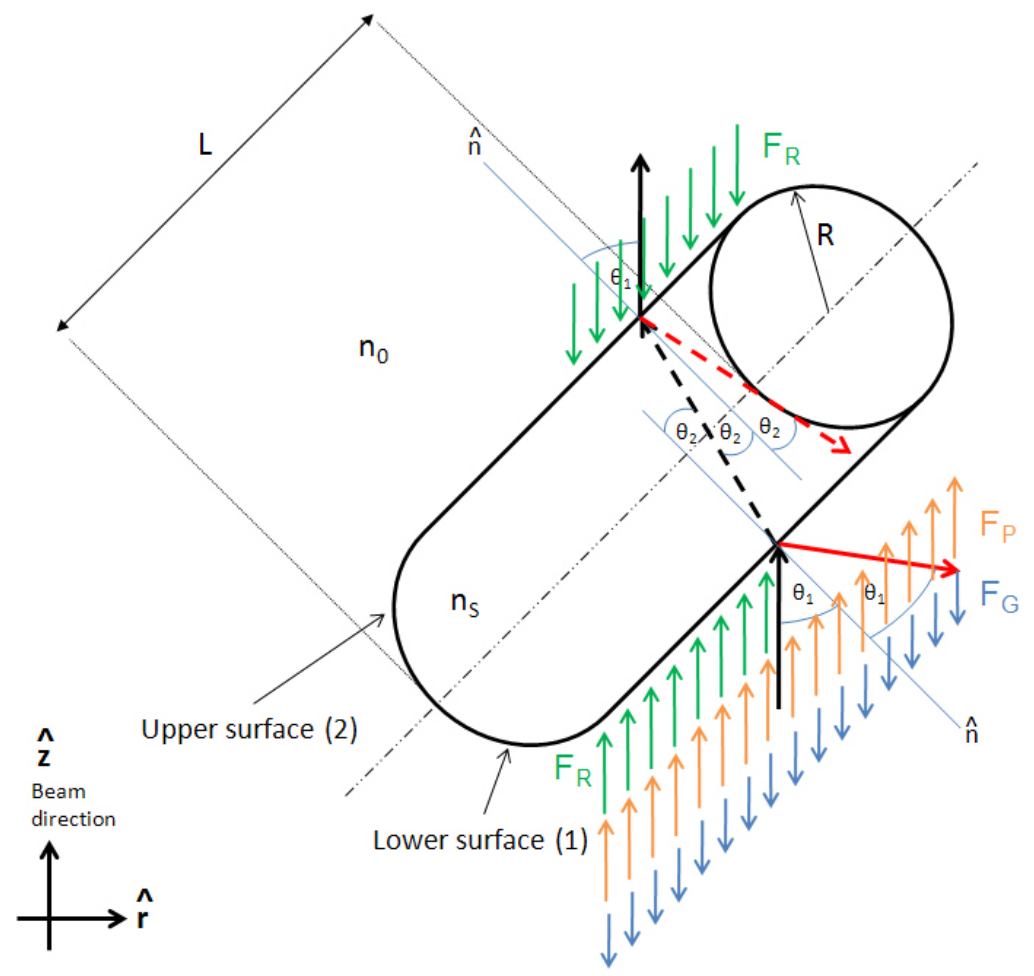

Figure A.1: Schematic representation of axial force field contributions. FP, FG, and FR indicate the forces due to photon scattering, gravity, and drag, respectively. 


\section{Appendix A.1. Scattering force}

Mathematically, the mechanical response of an object, situated in a beam of light, may be ex- pressed in terms of Newton's second law:

$$
F=\frac{d p}{d t}
$$

where $F$ is the force acting on the object, $d p$ the object's overall momentum change due to all pho- ton interactions, and $d t$ the short interval of time over which $d p$ is measured.

The momentum, $p$ of a single photon was formulated by de Broglie, who related particle- and wave-like properties in the equation

$$
p=\frac{h}{\lambda_{0}} n
$$

where $h$ is Planck's constant, $77 \lambda_{0}$ the wavelength of light in vacuum, and $n$ the refractive index associated with the lightwave's propagation in the medium.

(A) The total axial force, Fz acting on the particle may be expressed as the sum of four in- dependent contributions,

$$
F_{z}=F_{1 \tau z}+F_{1 t z}+F_{2 \tau z}+F_{2 t z}
$$

While $F_{1 \mathrm{rz}}$ accounts for the reflection of photons at the cylinder's lower surface, $F_{1 \mathrm{tz}}$ signifies the resultant force component associated with refraction; similarly, $F_{2 r z}$ and $F_{2 t z}$ represent the reflection and refraction contributions at the upper surface. To further define the terms above, a mathematical formulation must first be found describing the momentum transfer to the particle upon re- flection and/or refraction of a single photon at either surface. Using geometrical arguments from Figure A.2, the following relations may be postulated:

$$
\begin{aligned}
\Delta p_{1 r z}= & \frac{h}{\lambda_{0}} n_{0}\left[1+\cos \left(2 \theta_{1}\right)\right], \\
\Delta p_{1 t z}= & \frac{h}{\lambda_{0}}\left[n_{0}-n_{s} \cos \left(\theta_{1}-\theta_{2}\right)\right], \\
\Delta p_{2 r z}= & \frac{h}{\lambda_{0}} n_{s}\left[\cos \left(\theta_{1}-\theta_{2}\right)\right. \\
& \left.+\cos \left(3 \theta_{2}-\theta_{1}\right)\right], \\
\Delta p_{2 t z}= & \frac{h}{\lambda_{0}}\left\{n_{s} \cos \left(\theta_{1}-\theta_{2}\right)\right. \\
& \left.-n_{0} \cos \left[2\left(\theta_{1}-\theta_{2}\right)\right]\right\} .
\end{aligned}
$$



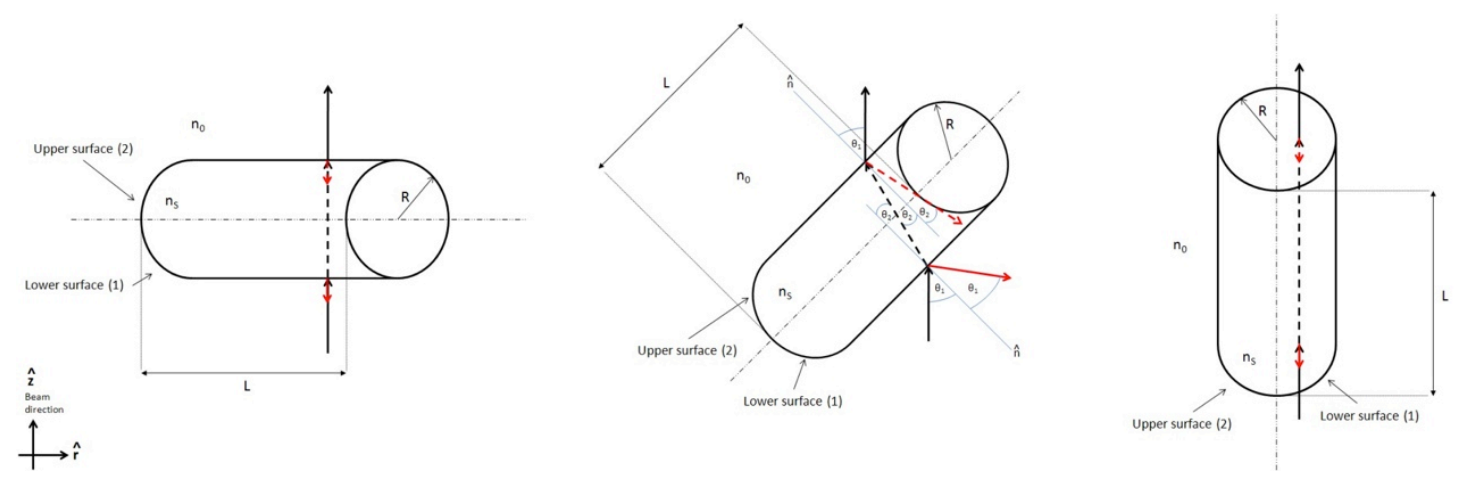

Figure A2: Ray diagram schematically illustrating the optical levitation of cylindrical objects inclined at an angle of (a) $0 \mathrm{rad}$, (b) $\theta_{1} \mathrm{rad}$, and (c) $\pi / 2 \mathrm{rad}$.

As before, the indices $z, 1,2, r$, and t stand for axial direction, lower surface, upper surface, re- flection, and refraction, respectively.

(B) The number of photons acting on the cylinder's outer surface is directly dependent on the physical characteristics of the light beam. Mathematically, the relation between these quan- tities is expressed in terms of the beam intensity profile, I. For the purposes of this study, the beam intensity profile may be approximated as that of the first order Gaussian mode

$$
I(\rho, z)=\frac{2 P}{\pi W(z)^{2}} \exp \left[\frac{-2 \rho^{2}}{W(z)^{2}}\right]
$$

where $\rho$ is the radial distance from the beam's central axis and $P$ is the total power associated with the beam. $W(z)$ describes the beam's width as a function of distance, $z$ away from the beam's minimum waist location:

$$
W(z)=W_{0}\left[1+\left(\frac{z}{z_{0}}\right)^{2}\right]^{\frac{1}{2}}
$$

where $z$ is taken to be positive in the direction of light propagation. $W_{0}$ is given by

$$
W_{0}=\left(\frac{\lambda_{0} z_{0}}{\pi}\right)^{\frac{1}{2}}
$$

where $z_{0}$ specifies the Rayleigh length or the position along the beam's axis at which $I(\rho, z)=I_{\max } / 2$.

(C) The power, $P$ in the light beam may be written as

$$
P=\Phi_{a v} E_{p h}
$$

with 


$$
\Phi_{a v}=\frac{N}{d t}
$$

and

$$
E_{p h}=\frac{h c}{\lambda_{0}}
$$

Here, $N$ represents the total number of photons, while $c$ stands for the speed of light in free space. $79 \Phi_{a v}$ and $E_{p h}$ refer to the average photon flux and the energy carried by a single photon, respectively.

(D) The fraction of photons reflected and transmitted at the interface between materials are given by Fresnel's equations. The associated power coefficients may, in the present case, be written as:

$$
\left|r_{1}\right|^{2}=\frac{Q_{1}}{\left\{Q_{2}+Q_{3}\right\}^{2}}
$$

with

$$
\begin{gathered}
Q_{1}=\left(n_{0} n_{s}\right)^{2}\left[\cos ^{2}\left(\theta_{1}\right)-\cos ^{2}\left(\theta_{2}\right)\right]^{2} \\
Q_{2}=n_{0} n_{s}\left[\cos ^{2}\left(\theta_{1}\right)+\cos ^{2}\left(\theta_{2}\right)\right] \\
Q_{3}=\left(n_{0}{ }^{2}+n_{s}{ }^{2}\right) \cos \left(\theta_{1}\right) \cos \left(\theta_{2}\right)
\end{gathered}
$$

and

$$
\left|t_{1}\right|^{2}=1-\left|r_{1}\right|^{2}
$$

where $|r|^{2}$ and $\left|t_{1}\right|^{2}$ represent the total power reflection and transmission coefficients, respectively.

Using the results from $(A)$ and $(D)$, the total change of momentum of the particle in the axial direction due to its interaction with a single photon can be written in the form:

$$
\begin{aligned}
d p_{z}= & \left|r_{1}\right|^{2} \Delta p_{1 r z}+\left|t_{1}\right|^{2} \Delta p_{1 t z}+\left|r_{1}\right|^{2} \\
& \times\left|t_{1}\right|^{2} \Delta p_{2 r z}+\left|t_{1}\right|^{2}\left|t_{1}\right|^{2} \Delta p_{2 t z}
\end{aligned}
$$

At the same time, the net axial force acting on the particle may be expressed in terms of three fundamental interactions, namely photon scattering, gravity, and drag:

$$
F_{\text {axial net }}=F_{z}-F_{g}-F_{d}
$$


While the subtractive terms above may readily be postulated as

$$
F_{g}=V\left(\rho_{s}-\rho_{0}\right) g
$$

and

$$
F_{g}=\frac{1}{2} \rho_{0} v^{2} C_{D} A
$$

the scattering component, $F_{z}$ may now be defined as the linear combination of four independent surface integrals, which are given by:

$$
\begin{aligned}
F_{1 r z}= & \int_{-\pi}^{0} \int_{0}^{L} \frac{n_{0}}{c}\left[1+\cos \left(2 \theta_{1}\right)\right] \\
& \times I(\rho, z)\left|r_{1}\right|^{2} R d s d \phi, \\
F_{1 t z}= & \int_{-\pi}^{0} \int_{0}^{L} \frac{1}{c}\left[n_{0}-n_{s} \cos \left(\theta_{1}-\theta_{2}\right)\right] \\
& \times I(\rho, z)\left|t_{1}\right|^{2} R d s d \phi, \\
F_{2 r z}= & \int_{-\pi}^{0} \int_{0}^{L} \frac{n_{s}}{c}\left[\cos \left(\theta_{1}-\theta_{2}\right)\right. \\
& \left.+\cos \left(3 \theta_{2}-\theta_{1}\right)\right] I(\rho, z)\left|r_{1}\right|^{2} \\
& \times\left|t_{1}\right|^{2} R d s d \phi,
\end{aligned}
$$

and

$$
\begin{aligned}
F_{2 t z}= & \int_{-\pi}^{0} \int_{0}^{L} \frac{1}{c}\left\{n_{s} \cos \left(\theta_{1}-\theta_{2}\right)\right. \\
& \left.-n_{0} \cos \left[2\left(\theta_{1}-\theta_{2}\right)\right]\right\} I(\rho, z) \\
& \times\left|t_{1}\right|^{2}\left|t_{1}\right|^{2} R d s d \phi .
\end{aligned}
$$

$R, d s$, and $d \phi 80$ denote a transformation to cylindrical coordinates. 


\section{Appendix A.2. Gradient force}

As with the axial component, the total radial force, Fr acting on the particle may be expressed as

$$
F_{\tau}=F_{1 \tau \tau}+F_{1 t \tau}+F_{2 \tau \tau}+F_{2 t \tau}
$$

Using Figure A2, a subsequent geometrical analysis yields

$$
\begin{aligned}
\Delta p_{1 r r}= & -\frac{h}{\lambda_{0}} n_{0} \sin \left(2 \theta_{1}\right) \cos (\psi), \\
\Delta p_{1 t r}= & \frac{h}{\lambda_{0}} n_{s} \sin \left(\theta_{1}-\theta_{2}\right) \cos (\psi), \\
\Delta p_{2 r r}= & \frac{h}{\lambda_{0}} n_{s}\left[\sin \left(3 \theta_{2}-\theta_{1}\right)\right. \\
& \left.-\sin \left(\theta_{1}-\theta_{2}\right)\right] \cos (\psi), \\
\Delta p_{2 t r}= & \frac{h}{\lambda_{0}}\left\{n_{0} \sin \left[2\left(\theta_{1}-\theta_{2}\right)\right]\right. \\
& \left.-n_{s} \sin \left(\theta_{1}-\theta_{2}\right)\right\} \cos (\psi) .
\end{aligned}
$$

A new angle, $\Psi$ is introduced to map the momentum's radial direction onto the unit vector, $\hat{r}$ defined in Figure 7 . Analogous to previous argumentation,

$$
\begin{aligned}
d p_{r}= & \left|r_{1}\right|^{2} \Delta p_{1 r r}+\left|t_{1}\right|^{2} \Delta p_{1 t r}+\left|r_{1}\right|^{2} \\
& \times\left|t_{1}\right|^{2} \Delta p_{2 r r}+\left|t_{1}\right|^{2}\left|t_{1}\right|^{2} \Delta p_{2 t r} .
\end{aligned}
$$

Not being affected by gravity, the net radial force is simply given by

$$
F_{\text {radial net }}=F_{\tau}-F_{d}
$$

Again, the scattering component, $F_{\tau}$ is obtained in terms of independent surface integrals of polar form: 


$$
\begin{aligned}
F_{1 r r}= & -\int_{-\pi}^{0} \int_{0}^{L} \frac{n_{0}}{c} \sin \left(2 \theta_{1}\right) \cos (\psi) \\
& \times I(\rho, z)\left|r_{1}\right|^{2} R d s d \phi, \\
F_{1 t r}= & \int_{-\pi}^{0} \int_{0}^{L} \frac{n_{s}}{c} \sin \left(\theta_{1}-\theta_{2}\right) \cos (\psi) \\
& \times I(\rho, z)\left|t_{1}\right|^{2} R d s d \phi, \\
F_{2 r r}= & \int_{-\pi}^{0} \int_{0}^{L} \frac{n_{s}}{c}\left[\sin \left(3 \theta_{2}-\theta_{1}\right)\right. \\
& \left.-\sin \left(\theta_{1}-\theta_{2}\right)\right] \cos (\psi) \\
& \times I(\rho, z)\left|r_{1}\right|^{2}\left|t_{1}\right|^{2} R d s d \phi, \\
F_{2 t r}= & \int_{-\pi}^{0} \int_{0}^{L} \frac{1}{c}\left\{n_{0} \sin \left[2\left(\theta_{1}-\theta_{2}\right)\right]\right. \\
& \left.-n_{s} \sin \left(\theta_{1}-\theta_{2}\right)\right\} \cos (\psi) \\
& \times I(\rho, z)\left|t_{1}\right|^{2}\left|t_{1}\right|^{2} R d s d \phi .
\end{aligned}
$$

It should be noted that $\rho=\rho\left(\theta_{1}\right)$ if the particle is centred in the Gaussian beam, but that $\rho=\rho\left(\theta_{1}, \psi\right)$ in general. 


\section{Appendix A.3. Torque}

Given the particle's non-spherical geometry, a torque, $T$ is generated causing the rotation of the cylinder. To calculate this torque, the mathematical equation

$$
\tau=\int_{S} \mathbf{r} \times d \mathbf{F}_{\substack{\text { axial } \\ \text { net }}}+\int_{S} \mathbf{r} \times d \mathbf{F}_{\text {radial }}^{\text {net }}
$$

may be used. In the above expression, $S$ describes the area over which momentum is transferred, $r$ defines a central reference point,

$$
d \mathbf{F}_{\substack{\text { axial } \\ \text { net }}}=\frac{I(\rho, z)}{E_{p h}} d p_{z} d A \hat{\mathbf{e}_{\mathbf{z}}}
$$

and

$$
d \mathbf{F}_{\text {radial }}^{\text {net }}=\frac{I(\rho, z)}{E_{p h}} d p_{r} d A \hat{\mathbf{e}_{\mathbf{r}}}
$$

The differential element, $d A$ is representative of a small surface element along the object's bottom face. In case the light beam's central axis coincides with either of the particle's axes of symmetry, the torque will vanish. 


\section{Appendix B: Optical levitation of cylindrical: MATLAB development and experimental validation}

\section{Appendix B.1. MATLAB source code}

MATLAB source code was developed to calculate the dynamic behaviour of a cylindrically shaped object under the influence of an intense beam of light. Both the changes in linear and angular momentum due to light-matter interactions between the beam's photonic particles and the cylinder's outer surface are determined within the source code. Furthermore, any forces acting on the levitated object due to changes in momentum are evaluated. Following these computations, the progressive dynamic behaviour of the overall system is modelled. A number of assumptions are made to more readily enable coding of the present simulation, i.e.

- All particle reinforcements are rigid and cylindrical in shape

- The reinforcement phase is homogeneous

- For a given time interval, $d t$, the total displacement of each fibre can be expressed as the superposition of displacements due to linear and angular momentum changes.

- All photon/body collisions are perfectly elastic

- Reinforcements are free to translate but may only rotate about the cylinder's centre of mass

Using the optical field gradient developed by the piezoceramic crystal array, consisting of three sets of opposing ultrasonic drivers (T1 - T6) (as illustrated in Figure 3), the MATLAB source is able to predict the resultant acoustic field depending upon the source frequency. 


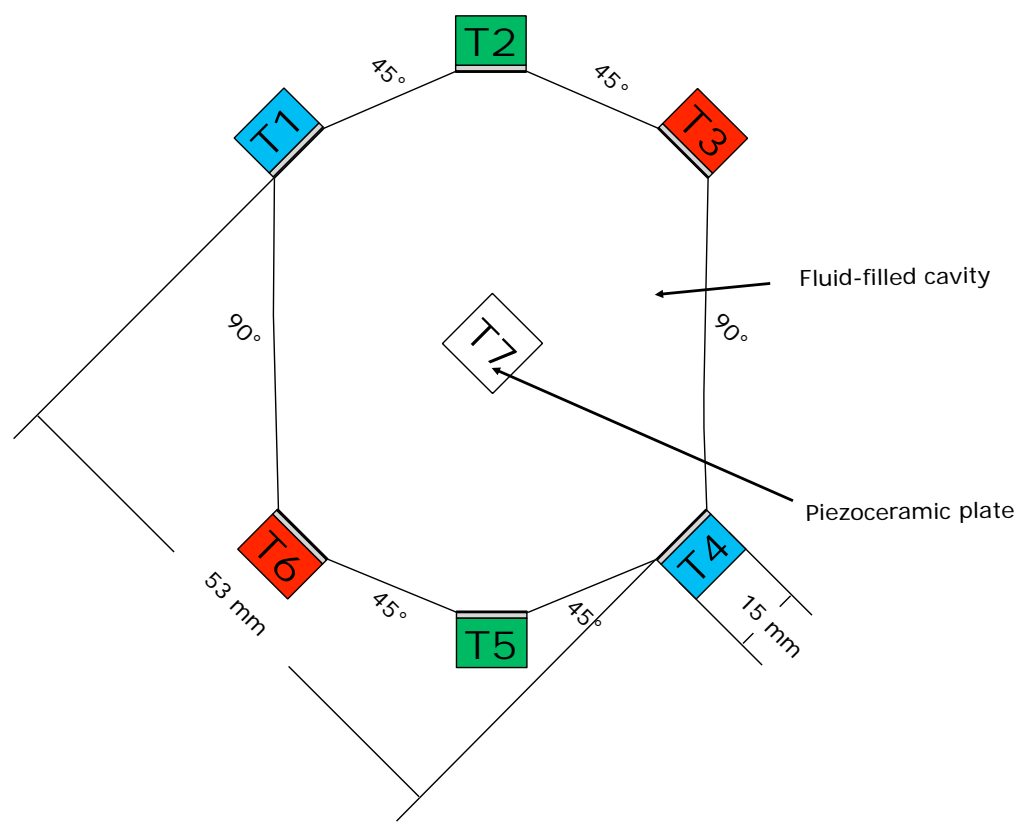

Figure B1.1: Device schematic. T1 - T6 mark six identical piezoelectric transducer elements. In addition, a small piezoceramic plate (T7) provides particle levitation capabilities. The maximum trapping area was restricted to $0.225 \mathrm{~mm} 2$.

Representative predictions and corresponding experimental results (viewed as micrographs) for the interference of plane counter-propagating travelling waves at 2.300 MHz for a single set of opposing transducers pair (T3 and T6) and two sets of opposing transducers pairs $(\mathrm{T} 1, \mathrm{~T} 4$ \& $\mathrm{T} 3, \mathrm{~T} 6)$ are presented in Figure $\mathrm{B} 1.2$. The colour scale indicates absolute pressure. The total amplitude is normalised to the maximum attainable pressure for the present device configuration (see Figure B1.1). The $x$ - and $y$-dimensions are measured in $[\mu \mathrm{m}]$.

Figure B1.3 (top-left) indicates an alternative acoustic pressure amplitude interference pattern when the T2 \& T5 transducers emit a set of plane counterpropagating travelling waves at $2.300 \mathrm{MHz}$. The matching experimental results are provided (top-right). Figure B1.3 (bottom) shows the corresponding result for the prediction and experimental results for $\mathrm{T} 2, \mathrm{~T} 5$ and $\mathrm{T} 3, \mathrm{~T} 6$ matched pairs with a plane counter-propagating travelling waves at $2.300 \mathrm{MHz}$ 

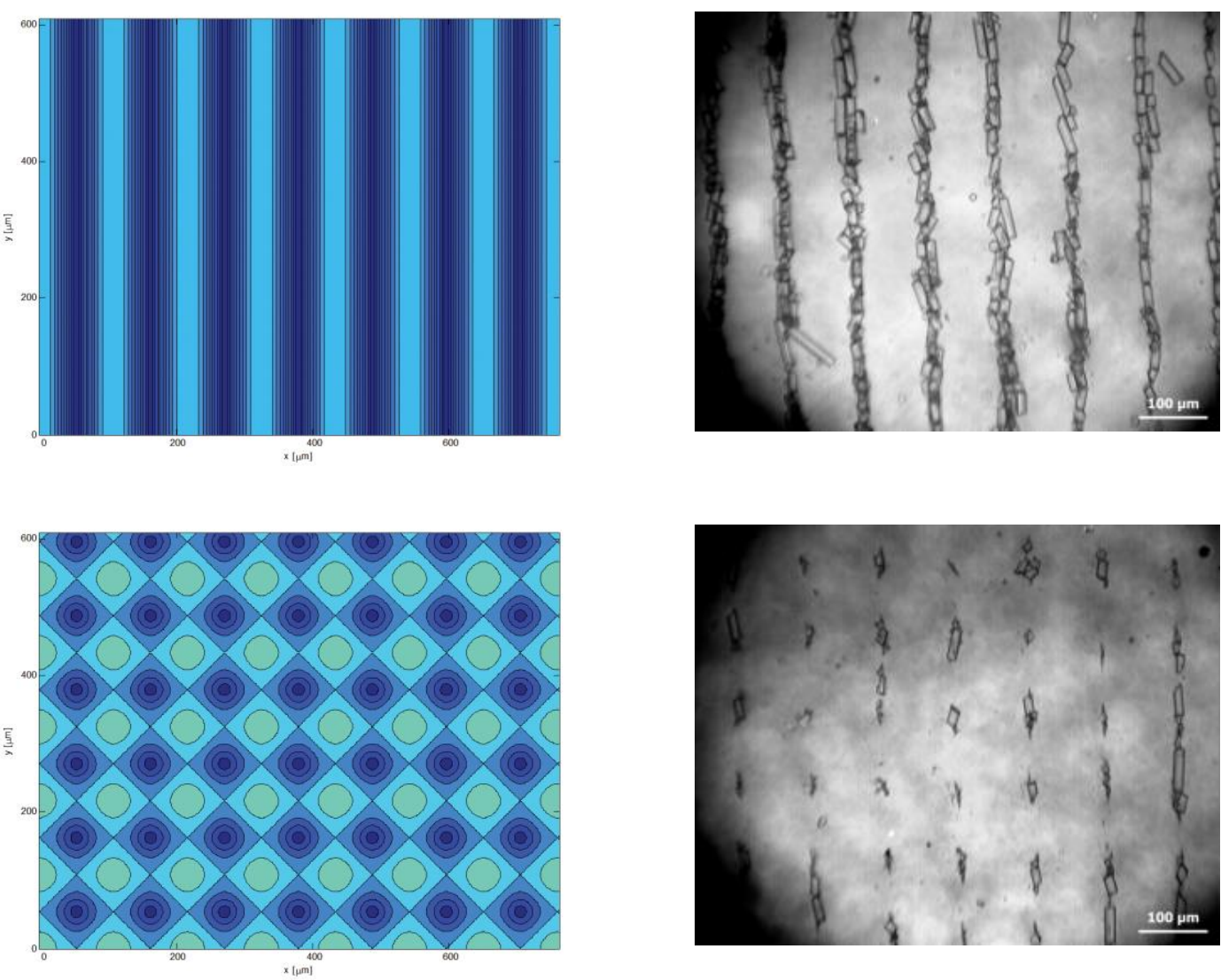

Figure B1.2: Prediction and experimental results for the interference of two plane counter-propagating travelling waves at $2.300 \mathrm{MHz}$. (Top Left) Acoustic pressure amplitude for a single set of opposing transducers pair (T3 and T6) with matching experimental result (Top Right). (Bottom Left) Acoustic pressure amplitude for two sets of opposing transducers pairs (T1, T4 \& T3, T6) with matching experimental result (Bottom Right)

Distribution A: Approved for public release; distribution is unlimited. 

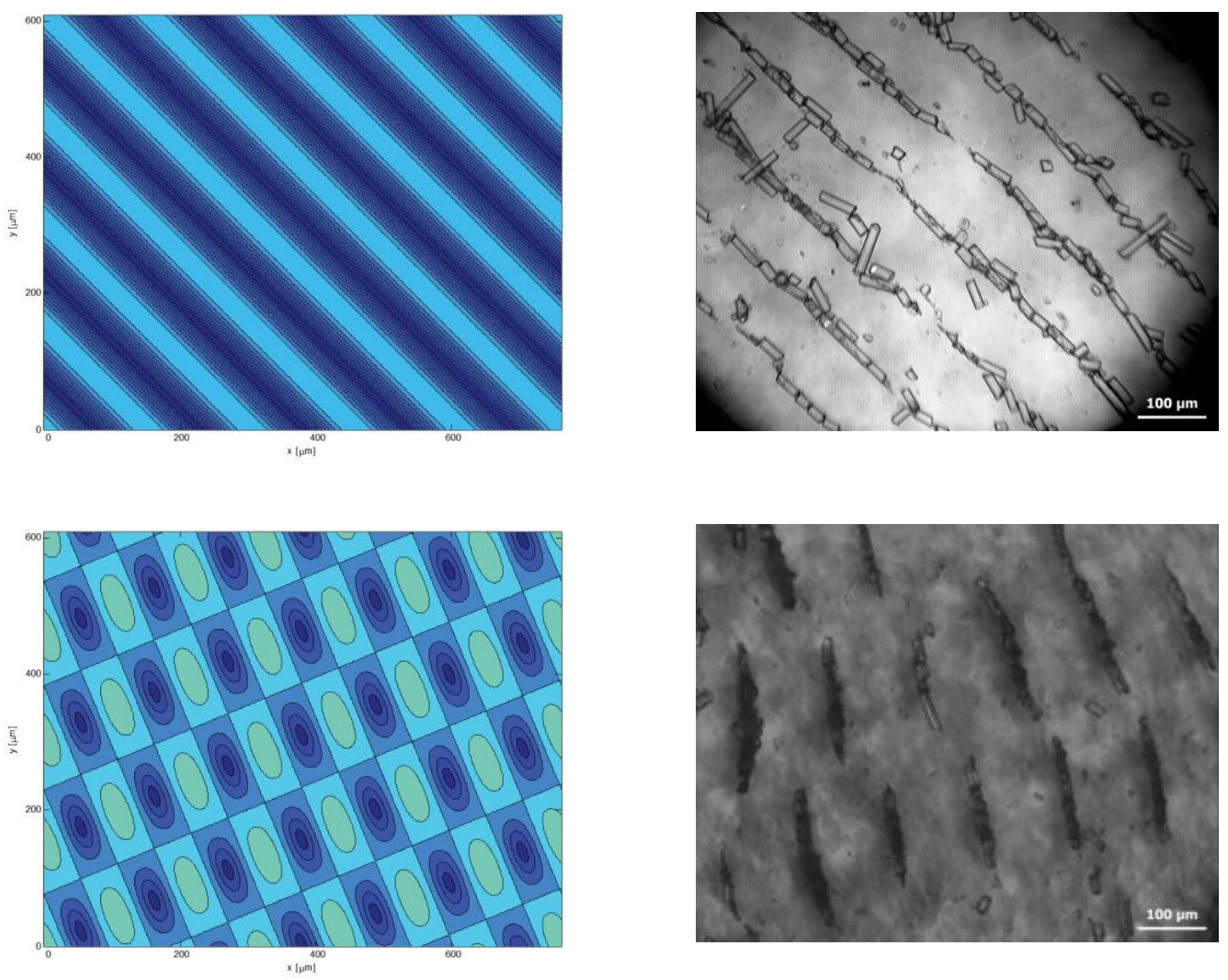

Figure B1.3: Prediction and experimental results for the interference of two plane counter-propagating travelling waves at 2.300MHz. (Top Left) Acoustic pressure amplitude for a single set of opposing transducers pair (T2 and T5) with matching experimental result (Top Right). (Bottom Left) Acoustic pressure amplitude for two sets of opposing transducers pairs $(T 2, T 5 \& T 3, T 6)$ with matching experimental result (Bottom Right)

Both figures show good agreement between the model and the experimental studies. However, it should be noted that no exact prediction can be made of the total number of particles that are drawn towards a single node but, to gain some indication, it may be possible to estimate the volume of low pressure and compare this with the particles' dimensions. In the case of larger particles, some form of 'bridging' across multiple nodes is to be expected, most likely following the path of least resistance. 